\title{
ON FULLY PRACTICAL FINITE ELEMENT APPROXIMATIONS OF DEGENERATE CAHN-HILLIARD SYSTEMS ${ }^{*, * *}$
}

\author{
John W. Barrett ${ }^{1}$, James F. Blowey ${ }^{2}$ and Harald Garcke ${ }^{3}$
}

\begin{abstract}
We consider a model for phase separation of a multi-component alloy with non-smooth free energy and a degenerate mobility matrix. In addition to showing well-posedness and stability bounds for our approximation, we prove convergence in one space dimension. Furthermore an iterative scheme for solving the resulting nonlinear discrete system is analysed. We discuss also how our approximation has to be modified in order to be applicable to a logarithmic free energy. Finally numerical experiments with three components in one and two space dimensions are presented.
\end{abstract}

Mathematics Subject Classification. 35K35, 35K55, 35K65, 65M12, 65M60, 82C26.

Received: April 7, 2000.

\section{INTRODUCTION}

It is the goal of this paper to develop and analyse a finite element approximation of the Cahn-Hilliard system with a degenerate mobility matrix. The Cahn-Hilliard system models phase separation and coarsening phenomena in multi-component systems. Examples are alloy or polymer systems consisting of $N$ different components. To understand the factors that influence phase separation and coarsening is of fundamental importance in applications. The material behaviour and in particular its lifetime drastically depends on how quickly the structure coarsens. Since it is difficult to obtain information by experiments reliable numerical simulations are very important.

If we denote by $u_{n}$ the fractional concentration of the $n$th component physical meaningful values of the vector $\boldsymbol{u}=\left(u_{1}, \ldots, u_{n}\right)^{T}$ have to be nonnegative and to fulfill the constraint

$$
\sum_{n=1}^{N} u_{n}=1
$$

Keywords and phrases. Phase separation, multi-component systems, degenerate parabolic systems of fourth order, finite element method, convergence analysis.

* Partially supported by the DFG through SFB256 Nichtlineare Partielle Differentialgleichungen.

** Partially supported by the EPSRC, UK through grants GR/M29689 and GR/M30951.

${ }^{1}$ Department of Mathematics, Imperial College, London, SW7 2BZ, UK. e-mail: j.barrett@ic.ac.uk

2 Department of Mathematical Sciences, University of Durham, DH1 3LE, UK.

${ }^{3}$ Institut für Angewandte Mathematik, Wegelerstraße 6, 53115 Bonn, Germany. 
One derives from the mass balance law that the individual components $u_{n}$ fulfill the continuity equation

$$
\frac{\partial}{\partial t} u_{n}+\nabla \cdot J_{n}=0,
$$

where the fluxes $J_{n}$ have to be determined. Neglecting the constraint (1.1) for the moment and assuming that the system is purely frictional and driven by chemical potential gradients, $\nabla w_{n}$, (see [29]) one obtains

$$
J_{n}=-l_{n}\left(u_{n}\right) \nabla w_{n},
$$

where typically $l_{n}\left(u_{n}\right):=D_{n} u_{n}$ with $D_{n} \in \mathbb{R}$ being the bare mobility of the $n$th component. In order for the constraint (1.1) to be fulfilled during the evolution [16] (see also [29]) proposed that the fluxes $J_{n}$ fulfill

$$
\sum_{n=1}^{N} J_{n}=0
$$

and that this is achieved through the presence of a force $F$ which is such that the modified fluxes

$$
J_{n}=l_{n}\left(u_{n}\right)\left(-\nabla w_{n}-F\right)
$$

fulfill the incompressibility condition (1.3). Using (1.3) and (1.4) we obtain that

$$
F=-\left(\sum_{n=1}^{N} l_{n}\left(u_{n}\right)\right)^{-1} \sum_{p=1}^{N} l_{p}\left(u_{p}\right) \nabla w_{p}
$$

Hence,

$$
\frac{\partial}{\partial t} \boldsymbol{u}=\nabla \cdot(L(\boldsymbol{u}) \nabla \boldsymbol{w}),
$$

where $L$ is a $N \times N$ mobility matrix and has the form

$$
\{L(\boldsymbol{u})\}_{n p} \equiv L_{n p}(\boldsymbol{u}):=l_{n}\left(u_{n}\right)\left(\delta_{n p}-\left(\sum_{q=1}^{N} l_{q}\left(u_{q}\right)\right)^{-1} l_{p}\left(u_{p}\right)\right)
$$

where $\delta_{n p}$ is the Kronecker delta.

The chemical potentials $\boldsymbol{w}=\left(w_{1}, \ldots, w_{N}\right)^{T}$ will be defined as the variational derivative of the GinzburgLandau free energy

$$
\mathcal{E}(\boldsymbol{u}):=\int_{\Omega}\left(\frac{\gamma}{2}|\nabla \boldsymbol{u}|^{2}+\Psi(\boldsymbol{u})\right) \mathrm{d} \boldsymbol{x},
$$

where $\Omega$ is a bounded domain in $\mathbb{R}^{d}, d \leq 3, \gamma>0$ is the gradient energy coefficient and

$$
\Psi(\boldsymbol{u}):=\Psi_{1}(\boldsymbol{u})-\frac{1}{2} \boldsymbol{u}^{T} A \boldsymbol{u}
$$

is the homogeneous free energy density. Here, $A$ is a constant symmetric $N \times N$ matrix taking into account the interaction between different components and the term $\Psi_{1}$ represents the entropy of the system and usually is 
taken to be of the form

$$
\Psi_{1}(\boldsymbol{u}):=\theta \sum_{n=1}^{N} u_{n} \ln u_{n} \quad \text { with absolute temperature } \theta>0
$$

or

$$
\Psi_{1}(\boldsymbol{u}):= \begin{cases}0 & \text { if } \boldsymbol{u} \in \mathbb{Q}^{N} \\ \infty & \text { elsewhere }\end{cases}
$$

To define the second expression, which is the deep quench limit of the logarithmic $\Psi_{1}$ (see [10]), we used the Gibbs simplex

$$
\mathbb{Q}^{N}:=\{\boldsymbol{\zeta} \in \mathcal{M}(1): \boldsymbol{\zeta} \geq \mathbf{0}\}, \quad \text { where } \forall \mu \in \mathbb{R} \quad \mathcal{M}(\mu):=\left\{\boldsymbol{\zeta} \in \mathbb{R}^{N}: \sum_{n=1}^{N} \zeta_{n}=\mu\right\}
$$

which is set of all physical meaningful values for the concentration vector.

Now the chemical potentials $w_{n}$ are defined as the variational derivative of $\mathcal{E}$ with respect to $u_{n}$, i.e. $w_{n}=\frac{\delta \mathcal{E}}{\delta u_{n}}$ which implies

$$
\boldsymbol{w}=-\gamma \Delta \boldsymbol{u}+\boldsymbol{D} \Psi_{1}(\boldsymbol{u})-A \boldsymbol{u}
$$

where $\left\{\boldsymbol{D} \Psi_{1}(\boldsymbol{u})\right\}_{n}:=\frac{\partial \Psi_{1}}{\partial u_{n}}(\boldsymbol{u})$. (1.10), together with equation (1.5), has to hold in $\Omega_{T}:=\Omega \times(0, T)$ where $T>0$ is an arbitrary but fixed time. We supplement this system with the boundary and initial conditions

$$
\begin{array}{cl}
\frac{\partial}{\partial \boldsymbol{\nu}} \boldsymbol{u}=L(\boldsymbol{u}) \frac{\partial}{\partial \boldsymbol{\nu}} \boldsymbol{w}=\mathbf{0} & \text { on } \partial \Omega \times(0, T), \\
\boldsymbol{u}(\boldsymbol{x}, 0)=\boldsymbol{u}^{0}(\boldsymbol{x}) & \forall \boldsymbol{x} \in \Omega
\end{array}
$$

with $\boldsymbol{\nu}$ being normal to $\partial \Omega$ where $\partial \Omega$ is assumed to be of Lipschitz class. On the initial data we make the following assumptions

$$
\text { a) } \mathbf{0} \leq \boldsymbol{u}^{0}(\boldsymbol{x}) \quad \text { and } \quad \text { b) } \quad N \sum \boldsymbol{u}^{0}(\boldsymbol{x})=1 \quad \forall \boldsymbol{x} \in \Omega \text {. }
$$

Here and throughout we write $\zeta_{n}$ for the $n$th component of $\zeta$ and set

$$
\sum \zeta:=\frac{1}{N} \sum_{n=1}^{N} \zeta_{n}, \quad\left(I-1 \sum\right) \zeta:=\zeta-1 \sum \zeta
$$

where $\mathbf{1} \in \mathbb{R}^{N}$ is defined by $\{\mathbf{1}\}_{n}=1, n=1 \rightarrow N$.

We note that the boundary conditions ensure that

$$
\frac{\mathrm{d}}{\mathrm{d} t} \int_{\Omega} \boldsymbol{u} \mathrm{d} \boldsymbol{x}=0 \quad \text { and } \quad \frac{\mathrm{d}}{\mathrm{d} t} \mathcal{E}(\boldsymbol{u}) \leq 0
$$

Hence, the total mass is conserved and the free energy $\mathcal{E}$ serves as a Lyapunov functional for the system. The discrete analogue of these two properties will be of major importance when we analyse the finite element approximation.

Cahn-Hilliard systems to model phase separation in multicomponent systems were first studied by Morral and Cahn [27] and de Fontaine [15]. An existence result in the case of a constant mobility matrix $L$ has been first given by Elliott and Luckhaus [20]. The fact that Cahn-Hilliard systems model phase separation phenomena in 
multicomponent systems was supported by numerical simulations performed by Eyre [21], Blowey et al. [9] and Barrett and Blowey [2]. The latter paper also derives error bounds for a finite element approximation.

All the papers mentioned so far consider the case of a constant mobility matrix. But as we have seen in the derivation of the Cahn-Hilliard system above the mobility matrix in general is concentration dependent (see (1.6)). In the following we will assume that the individual mobility functions $l_{n} \in C([0,1])$ fulfill for some $l_{\min }, l_{\max } \in \mathbb{R}^{+}$

$$
l_{\text {min }} s \leq l_{n}(s) \leq l_{\max } s \quad \forall s \in[0,1] .
$$

It follows from (1.6) and (1.13) that for all $\zeta \in \mathbb{Q}^{N}$

$$
[L(\boldsymbol{\zeta})]^{T} \equiv L(\boldsymbol{\zeta}), \quad L(\boldsymbol{\zeta}) \mathbf{1}=\mathbf{0}
$$

and for all $\boldsymbol{\eta} \in \mathbb{R}^{N}$

$$
\begin{aligned}
\boldsymbol{\eta}^{T} L(\boldsymbol{\zeta}) \boldsymbol{\eta} & \equiv \sum_{n=1}^{N} \sum_{p=1}^{N} \eta_{n} L_{n p}(\boldsymbol{\zeta})\left(\eta_{p}-\eta_{n}\right) \equiv-\sum_{n=2}^{N} \sum_{p=1}^{n-1} L_{n p}(\boldsymbol{\zeta})\left(\eta_{n}-\eta_{p}\right)^{2} \\
& \equiv\left(\mathbf{1}^{T} \boldsymbol{l}(\boldsymbol{\zeta})\right)^{-1} \sum_{n=2}^{N} \sum_{p=1}^{n-1} l_{n}\left(\zeta_{n}\right) l_{p}\left(\zeta_{p}\right)\left(\eta_{n}-\eta_{p}\right)^{2}
\end{aligned}
$$

where $\mathbf{0} \in \mathbb{R}^{N}$ and $\boldsymbol{l}(\boldsymbol{\zeta})$ are defined by

$$
\{\boldsymbol{0}\}_{n}:=0, \quad \text { and } \quad\{\boldsymbol{l}(\boldsymbol{\zeta})\}_{n}:=l_{n}\left(\zeta_{n}\right), \quad n=1 \rightarrow N .
$$

Equations $(1.14 \mathrm{a}, \mathrm{b})$ show that the matrix $L(\boldsymbol{\zeta})$ is positive semi-definite for all $\boldsymbol{\zeta} \in \mathbb{Q}^{N}$ with $\mathbf{1}$ an eigenvector with eigenvalue zero. As the system is solved on $\mathcal{M}(1)$ a degeneracy in a direction orthogonal to the corresponding tangent space $\mathcal{M}(0)$ is of no relevance for the analysis (see [19,20]). But equation (1.14b) and assumption (1.13) imply that $L$ also degenerates in tangential directions if one of the components becomes zero. Hence, the resulting equations (1.5) and (1.10) are a system of fourth order degenerate parabolic equations. Existence of weak solutions to the degenerate Cahn-Hilliard system has been shown by Elliott and Garcke [19] (see also [18]). But so far no uniqueness result is known for this type of systems. For an overview on the vast literature on mathematical results for the Cahn-Hilliard model we refer to Elliott [17] and Novick-Cohen [28].

In this paper a first attempt to numerically approximate solutions to the degenerate Cahn-Hilliard system is made. The numerical method uses continuous piecewise linear finite elements to discretise $\boldsymbol{u}$ and $\boldsymbol{w}$ in space and uses essentially an implicit Euler scheme for the time discretisation. The ideas to numerically treat the degeneracy of the system is based on previous work by the authors on scalar degenerate parabolic equations of fourth order (see $[7,8]$ ). We also make use of ideas introduced by Barrett and Blowey who studied finite element approximations for Cahn-Hilliard systems with a concentration dependent but non-degenerate mobility matrix $($ see $[4,5])$. We refer also to the work of Zhornitskaya and Bertozzi [30] and Grün and Rumpf [24] who have also developed numerical methods for approximating scalar degenerate parabolic equations of fourth order. Their approach makes use of entropy type estimates (see also Th. 2.3 in this paper).

The outline of this paper is as follows. After introducing some notation and some auxiliary results we introduce a fully practical finite element approximation for the degenerate Cahn-Hilliard system in the deep quench limit. We show stability bounds that hold in all space dimensions and convergence in the case of one space dimension. In addition, we discuss how entropy estimates, which give $H^{2}$-estimates for the concentration vector $\boldsymbol{u}$, can be obtained. The finite element approximation for the Cahn-Hilliard system with the deep quench limit potential leads to a discrete variational inequality. In Section 3 convergence of an algorithm for solving the discrete variational inequality is shown. In Section 4 we show how our method has to be modified in order to deal with a logarithmic free energy. Finally, we report on some numerical experiments in Section 5. In 
particular, we compare the numerical results to qualitative predictions of the asymptotic analysis of Garcke and Novick-Cohen [23]. The presented numerical results show the occurrence of chain like patterns and wetting phenomena are also observed.

\subsection{Notation and auxiliary results}

We have adopted the standard notation for Sobolev spaces, denoting the norm of $W^{m, p}(\Omega)(m \in \mathbb{N}, p \in[1, \infty])$ by $\|\cdot\|_{m, p}$ and the semi-norm by $|\cdot|_{m, p}$. For $p=2, W^{m, 2}(\Omega)$ will be denoted by $H^{m}(\Omega)$ with the associated norm and semi-norm written, as respectively, $\|\cdot\|_{m}$ and $|\cdot|_{m}$. Furthermore, we define $\boldsymbol{L}^{2}\left(\Omega_{T}\right):=L^{2}\left(0, T ; \boldsymbol{L}^{2}(\Omega)\right)$. For $\boldsymbol{\eta} \in \boldsymbol{H}^{1}(\Omega), \nabla \boldsymbol{\eta}$ denotes the $N \times d$ matrix with entries $\{\nabla \boldsymbol{\eta}\}_{n m}:=\frac{\partial}{\partial x_{m}} \eta_{n}$ and then $\frac{\partial}{\partial \boldsymbol{\nu}} \boldsymbol{\eta}:=(\nabla \boldsymbol{\eta}) \boldsymbol{\nu}$. Finally, for an $N \times d$ matrix $\Lambda$ with entries $\Lambda_{n m} \in H^{1}(\Omega), \nabla \cdot \Lambda$ is the $N \times 1$ vector with entries $\sum_{m=1}^{d} \frac{\partial}{\partial x_{m}} \Lambda_{n m}$, $n=1 \rightarrow N$. Throughout $(\cdot, \cdot)$ denotes the standard $L^{2}$ inner product over $\Omega$. This is naturally extended to vector and matrix functions, e.g. for $I \times J$ matrices $\Lambda(\boldsymbol{x})$ and $\Xi(\boldsymbol{x})$ with entries in $L^{2}(\Omega)$

$$
(\Lambda, \Xi):=\sum_{i=1}^{I} \sum_{j=1}^{J}\left(\Lambda_{i j}, \Xi_{i j}\right):=\sum_{i=1}^{I} \sum_{j=1}^{J} \int_{\Omega} \Lambda_{i j}(\boldsymbol{x}) \Xi_{i j}(\boldsymbol{x}) \mathrm{d} \boldsymbol{x} .
$$

In addition $\langle\cdot, \cdot\rangle$ denotes the duality pairing between $\left(H^{1}(\Omega)\right)^{\prime}$ and $H^{1}(\Omega)$, which is extended to vector functions in the standard way. We introduce the following convex sets:

$$
\begin{aligned}
\boldsymbol{K} & :=\left\{\boldsymbol{\eta} \in \boldsymbol{H}^{1}(\Omega): \boldsymbol{\eta}(\boldsymbol{x}) \geq \mathbf{0} \quad \text { for a.e. } \boldsymbol{x} \in \Omega\right\}, \\
\boldsymbol{K}_{\boldsymbol{m}} & :=\left\{\boldsymbol{\eta} \in \boldsymbol{K}: \boldsymbol{f} \boldsymbol{\eta}=\boldsymbol{m}:=f \boldsymbol{u}^{0} \text { and } \boldsymbol{\eta}(\boldsymbol{x}) \in \mathcal{M}(1) \text { for a.e. } \boldsymbol{x} \in \Omega\right\} ;
\end{aligned}
$$

where

$$
f \boldsymbol{\eta}:=\frac{1}{|\Omega|} \int_{\Omega} \boldsymbol{\eta}(\boldsymbol{x}) \mathrm{d} \boldsymbol{x} \quad \forall \boldsymbol{\eta} \in \boldsymbol{L}^{2}(\Omega) .
$$

For later purposes, we recall the following well-known Sobolev interpolation results, e.g. see Adams and Fournier [1]: Let $p \in[1, \infty], m \geq 1$,

$$
r \in \begin{cases}{[p, \infty]} & \text { if } m-\frac{d}{p}>0 \\ {[p, \infty)} & \text { if } m-\frac{d}{p}=0 \\ {\left[p,-\frac{d}{m-(d / p)}\right]} & \text { if } m-\frac{d}{p}<0\end{cases}
$$

and $\mu=\frac{d}{m}\left(\frac{1}{p}-\frac{1}{r}\right)$. Then there is a constant $C$ depending only on $\Omega, p, r, m$ such that for all $v \in W^{m, p}(\Omega)$ the inequality

$$
\|v\|_{0, r} \leq C\|v\|_{0, p}^{1-\mu}\|v\|_{m, p}^{\mu}
$$

holds. It is convenient to introduce the "inverse Laplacian" operator $\mathcal{G}: \mathcal{F} \rightarrow V$ such that

$$
(\nabla \mathcal{G} v, \nabla \eta)=\langle v, \eta\rangle \quad \forall \eta \in H^{1}(\Omega),
$$

where

$$
\mathcal{F}:=\left\{v \in\left(H^{1}(\Omega)\right)^{\prime}:\langle v, 1\rangle=0\right\} \quad \text { and } \quad V:=\left\{v \in H^{1}(\Omega):(v, 1)=0\right\} .
$$

The well-posedness of $\mathcal{G}$ follows from the Lax-Milgram theorem and the Poincaré inequality

$$
|\eta|_{0, p} \leq C\left(|\eta|_{1, p}+|(\eta, 1)|\right) \quad \forall \eta \in W^{1, p}(\Omega) \quad \text { and } \quad p \in[1, \infty] .
$$


One can define a norm on $\mathcal{F}$ by

$$
\|v\|_{-1}:=|\mathcal{G} v|_{1} \equiv\langle v, \mathcal{G} v\rangle^{\frac{1}{2}} \quad \forall v \in \mathcal{F} .
$$

We note also for future reference that using a Young's inequality yields for all $\alpha>0$ that

$$
\langle v, \eta\rangle=(\nabla \mathcal{G} v, \nabla \eta) \leq\|v\|_{-1}|\eta|_{1} \leq \frac{1}{2 \alpha}\|v\|_{-1}^{2}+\frac{\alpha}{2}|\eta|_{1}^{2} \quad \forall v \in \mathcal{F}, \eta \in H^{1}(\Omega) .
$$

Throughout the paper, the norm $\|\cdot\|$ operating on matrices is that subordinate to the Euclidean vector norm, $\|\boldsymbol{\eta}\|^{2}:=\boldsymbol{\eta}^{T} \boldsymbol{\eta}$ for $\boldsymbol{\eta} \in \mathbb{R}^{N}$, i.e. the spectral radius for symmetric matrices. $C$ denotes a generic constant independent of $h, \tau, \sigma$ and $\varepsilon$, the mesh and temporal discretisation parameters and the regularisation parameters. In addition $C\left(a_{1}, \cdots, a_{I}\right)$ denotes a constant depending on the nonnegative parameters $\left\{a_{i}\right\}_{i=1}^{I}$, such that for all $C_{1}>0$ there exists a $C_{2}>0$ such that $C\left(a_{1}, \cdots, a_{I}\right) \leq C_{2}$ if $a_{i} \leq C_{1}$ for $i=1 \rightarrow I$. Finally, we define $\boldsymbol{e}^{n} \in \mathbb{R}^{N}, n=1 \rightarrow N$, by $e_{p}^{n}:=\delta_{n p}$.

\section{Finite element approximation}

Let $(\mathrm{P})$ denote the degenerate system (1.5), (1.10) and (1.11a,b). We consider the finite element approximation of the problem $(\mathrm{P})$ under the following assumptions on the meshes:

(A) Let $\Omega$ be a polyhedral domain. Let $\left\{\mathcal{T}^{h}\right\}_{h>0}$ be a quasi-uniform family of partitionings of $\Omega$ into disjoint open simplices $\kappa$ with $h_{\kappa}:=\operatorname{diam}(\kappa)$ and $h:=\max _{\kappa \in \mathcal{T}^{h}} h_{\kappa}$, so that $\bar{\Omega}=\cup_{\kappa \in \mathcal{T}^{h}} \bar{\kappa}$.

Associated with $\mathcal{T}^{h}$ is the finite element space

$$
S^{h}:=\left\{\chi \in C(\bar{\Omega}):\left.\chi\right|_{\kappa} \text { is linear } \forall \kappa \in \mathcal{T}^{h}\right\} \subset H^{1}(\Omega) .
$$

We extend this definition to vector functions, i.e. $\chi \in S^{h} \Rightarrow \chi_{n} \in S^{h}, n=1 \rightarrow N$. Let $J$ be the set of nodes of $\mathcal{T}^{h}$ and $\left\{\boldsymbol{x}_{j}\right\}_{j \in J}$ the coordinates of these nodes. Let $\left\{\beta_{j}\right\}_{j \in J}$ be the standard basis functions for $S^{h}$; that is $\beta_{j} \in K^{h}$ and $\beta_{j}\left(\boldsymbol{x}_{i}\right)=\delta_{i j}$ for all $i, j \in J$. We introduce $\pi^{h}: C(\bar{\Omega}) \rightarrow S^{h}$, the interpolation operator, such that $\pi^{h} \eta\left(\boldsymbol{x}_{j}\right)=\eta\left(\boldsymbol{x}_{j}\right)$ for all $j \in J$. A discrete semi-inner product on $C(\bar{\Omega})$ is defined by

$$
\left(\eta_{1}, \eta_{2}\right)^{h}:=\int_{\Omega} \pi^{h}\left(\eta_{1}(\boldsymbol{x}) \eta_{2}(\boldsymbol{x})\right) \mathrm{d} \boldsymbol{x} \equiv \sum_{j \in J} \omega_{j} \eta_{1}\left(\boldsymbol{x}_{j}\right) \eta_{2}\left(\boldsymbol{x}_{j}\right)
$$

where $\omega_{j}:=\left(1, \beta_{j}\right)$. The induced semi-norm is then $|\cdot|_{h}:=\left[(\cdot, \cdot)^{h}\right]^{\frac{1}{2}}$. We extend naturally the above definitions to vector functions; i.e. $\boldsymbol{\pi}^{h}: \boldsymbol{C}(\bar{\Omega}) \rightarrow \boldsymbol{S}^{h}$ with $\left\{\boldsymbol{\pi}^{h} \boldsymbol{\eta}\right\}_{n}:=\pi^{h} \eta_{n}$, then (2.1) is extended as in (1.15). We introduce the $L^{2}$ projections $\boldsymbol{Q}_{1}^{h}, \boldsymbol{Q}_{2}^{h}: \boldsymbol{L}^{2}(\Omega) \rightarrow \boldsymbol{S}^{h}$, where $\left\{\boldsymbol{Q}_{i}^{h} \boldsymbol{\eta}\right\}_{n}:=Q_{i}^{h} \eta_{n}$, and $Q_{1}^{h}, Q_{2}^{h}: L^{2}(\Omega) \rightarrow S^{h}$ are defined by

$$
\left(Q_{1}^{h} \eta, \chi\right)=\left(Q_{2}^{h} \eta, \chi\right)^{h}=(\eta, \chi) \quad \forall \chi \in S^{h} .
$$

Let $\psi_{1} \in C([0,1])$ be convex. We then introduce the homogeneous free energy density $\Psi \in \boldsymbol{C}\left([0,1]^{N}\right)$ defined by

$$
\Psi(\boldsymbol{\zeta}):=\Psi_{1}(\boldsymbol{\zeta})-\frac{1}{2} \boldsymbol{\zeta}^{T} A \boldsymbol{\zeta}, \quad \text { where } \quad \Psi_{1}(\boldsymbol{\zeta}):=\sum_{n=1}^{N} \psi_{1}\left(\zeta_{n}\right) .
$$

Obviously all the examples of $\Psi$ given in Section 1 can be written in this form. For example the logarithmic case corresponds to $\psi_{1}(s):=\theta s \ln s$, and the deep quench case to $\psi_{1} \equiv 0$. In this section and the next we assume that $\psi_{1} \in C^{1}([0,1])$. This obviously excludes the logarithmic case. Modifications to our approach will be made 
in Section 4 to cope with this case. For our numerical approximation of problem (P) we split $A \equiv A^{+}+A^{-}$, where $A^{+(-)}$is symmetric positive (negative) semi-definite.

In addition we regularise the degenerate mobility matrix $L$ by introducing $L^{\sigma}$, where for all $\sigma \in(0,1)$ and $\zeta \in \mathbb{Q}^{N}$

$$
\begin{aligned}
& \left\{L^{\sigma}(\boldsymbol{\zeta})\right\}_{n p} \equiv L_{n p}^{\sigma}(\boldsymbol{\zeta}):=l_{n}^{\sigma}\left(\zeta_{n}\right)\left(\delta_{n p}-\left(\mathbf{1}^{T} \boldsymbol{l}^{\sigma}(\boldsymbol{\zeta})\right)^{-1} l_{p}^{\sigma}\left(\zeta_{p}\right)\right) ; \\
& \text { and } \quad\left\{\boldsymbol{l}^{\sigma}(\boldsymbol{\zeta})\right\}_{n}:=l_{n}^{\sigma}\left(\zeta_{n}\right) \quad:=l_{n}\left(\zeta_{n}\right)+\sigma, \quad n=1 \rightarrow N .
\end{aligned}
$$

It follows from (2.4a,b), similarly to (1.14a,b), that for all $\zeta \in \mathbb{Q}^{N}$ and $\boldsymbol{\eta} \in \mathbb{R}^{N}$

$$
\begin{aligned}
& {\left[L^{\sigma}(\boldsymbol{\zeta})\right]^{T} \equiv L^{\sigma}(\boldsymbol{\zeta}), \quad L^{\sigma}(\boldsymbol{\zeta}) \mathbf{1}=\mathbf{0} \quad \text { and }} \\
& \boldsymbol{\eta}^{T} L^{\sigma}(\boldsymbol{\zeta}) \boldsymbol{\eta} \equiv\left(\mathbf{1}^{T} \boldsymbol{l}^{\sigma}(\boldsymbol{\zeta})\right)^{-1} \sum_{n=2}^{N} \sum_{p=1}^{n-1} l_{n}^{\sigma}\left(\zeta_{n}\right) l_{p}^{\sigma}\left(\zeta_{p}\right)\left(\eta_{n}-\eta_{p}\right)^{2} .
\end{aligned}
$$

If $\boldsymbol{\eta} \in \mathcal{M}(0)$, it follows that $\eta_{n}=\sum\left(\eta_{n} \mathbf{1}-\boldsymbol{\eta}\right), n=1 \rightarrow N$. Hence we have that

$$
\|\boldsymbol{\eta}\|^{2}=\frac{1}{N} \sum_{n=1}^{N} \sum_{p=1}^{N} \eta_{n}\left(\eta_{n}-\eta_{p}\right)=\frac{1}{N} \sum_{n=2}^{N} \sum_{p=1}^{n-1}\left(\eta_{n}-\eta_{p}\right)^{2} \quad \forall \boldsymbol{\eta} \in \mathcal{M}(0) .
$$

Therefore combining (2.5b), (2.4b), (1.13) and (2.6) we have that

$$
L_{\min }^{\sigma}\|\boldsymbol{\eta}\|^{2} \leq \boldsymbol{\eta}^{T} L^{\sigma}(\boldsymbol{\zeta}) \boldsymbol{\eta} \quad \forall \boldsymbol{\zeta} \in \mathbb{Q}^{N}, \quad \boldsymbol{\eta} \in \mathcal{M}(0) ;
$$

where $L_{\min }^{\sigma}:=N \sigma^{2} /\left(l_{\max }+N \sigma\right)$. In addition, it is easily established for all $\boldsymbol{\zeta} \in \mathbb{Q}^{N}$ and $\boldsymbol{\eta} \in \mathbb{R}^{N}$ that

$$
\begin{aligned}
\sum_{n=2}^{N} \sum_{p=1}^{n-1} l_{n}^{(\sigma)}\left(\zeta_{n}\right) l_{p}^{(\sigma)}\left(\zeta_{p}\right)\left(\eta_{n}-\eta_{p}\right)^{2} & \leq \sum_{n=1}^{N} \sum_{p \neq n} l_{n}^{(\sigma)}\left(\zeta_{n}\right) l_{p}^{(\sigma)}\left(\zeta_{p}\right)\left(\eta_{n}^{2}+\eta_{p}^{2}\right) \\
& =2 \sum_{n=1}^{N}\left(\sum_{p \neq n} l_{p}^{(\sigma)}\left(\zeta_{p}\right)\right) l_{n}^{(\sigma)}\left(\zeta_{n}\right) \eta_{n}^{2}
\end{aligned}
$$

In the above and throughout we adopt the notation $l_{n}^{(\sigma)}$, which is an abbreviation for either "with" or "without" the superscript $\sigma$. Therefore combining (1.14b), (2.5b), (2.4b), (1.13) and (2.8), we have that

$$
\boldsymbol{\eta}^{T} L^{(\sigma)}(\boldsymbol{\zeta}) \boldsymbol{\eta} \leq L_{\max }^{(\sigma)}\|\boldsymbol{\eta}\|^{2} \quad \forall \boldsymbol{\zeta} \in \mathbb{Q}^{N}, \quad \boldsymbol{\eta} \in \mathbb{R}^{N},
$$

where $L_{\max }:=\frac{1}{2} l_{\max }$ and $L_{\max }^{\sigma}:=\frac{1}{2}\left(l_{\max }+N \sigma\right)$.

For the finite element approximation of $(\mathrm{P})$ by $S^{h}$, we introduce

$$
\begin{aligned}
\boldsymbol{K}^{h} & :=\left\{\chi \in \boldsymbol{S}^{h}: \chi\left(\boldsymbol{x}_{j}\right) \geq \mathbf{0} \quad \forall j \in J\right\}, \\
\boldsymbol{K}_{\boldsymbol{m}}^{h} & :=\left\{\chi \in \boldsymbol{K}^{h}: f \boldsymbol{\chi}=\boldsymbol{m}:=f \boldsymbol{u}^{0} \text { and } \boldsymbol{\chi}\left(\boldsymbol{x}_{j}\right) \in \mathcal{M}(1) \quad \forall j \in J\right\} .
\end{aligned}
$$


Let $0 \equiv t_{0}<t_{1}<\cdots t_{K-1}<t_{K} \equiv T$ be a partitioning of $[0, T]$ into variable time steps $\tau_{k}:=t_{k}-t_{k-1}$, $k=1 \rightarrow K$. Let $\tau:=\max _{k=1 \rightarrow K} \tau_{k}$. Assuming that $\psi_{1} \in C^{1}([0,1])$, we then consider the following fully practical finite element approximation of $(\mathrm{P})$ :

$\left(\mathbf{P}_{\sigma}^{h, \tau}\right)$ For $k \geq 1$, find $\left\{\boldsymbol{U}_{\sigma}^{k}, \boldsymbol{W}_{\sigma}^{k}\right\} \in \boldsymbol{K}^{h} \times \boldsymbol{S}^{h}$ such that

$$
\begin{aligned}
& \left(\frac{\boldsymbol{U}_{\sigma}^{k}-\boldsymbol{U}_{\sigma}^{k-1}}{\tau_{k}}, \boldsymbol{\chi}\right)^{h}+\left(L^{\sigma}\left(\boldsymbol{U}_{\sigma}^{k-1}\right) \nabla \boldsymbol{W}_{\sigma}^{k}, \nabla \boldsymbol{\chi}\right)=0 \quad \forall \boldsymbol{\chi} \in \boldsymbol{S}^{h}, \\
& \gamma\left(\nabla \boldsymbol{U}_{\sigma}^{k}, \nabla\left(\boldsymbol{\chi}-\boldsymbol{U}_{\sigma}^{k}\right)\right)+\left(\boldsymbol{D} \Psi_{1}\left(\boldsymbol{U}_{\sigma}^{k}\right)-A^{-} \boldsymbol{U}_{\sigma}^{k}-\boldsymbol{W}_{\sigma}^{k}, \boldsymbol{\chi}-\boldsymbol{U}_{\sigma}^{k}\right)^{h} \geq\left(A^{+} \boldsymbol{U}_{\sigma}^{k-1}, \boldsymbol{\chi}-\boldsymbol{U}_{\sigma}^{k}\right)^{h} \quad \forall \boldsymbol{\chi} \in \boldsymbol{K}^{h} ;
\end{aligned}
$$

where $\boldsymbol{U}_{\sigma}^{0} \in \boldsymbol{K}_{\boldsymbol{m}}^{h}$ is an approximation of $\boldsymbol{u}^{0} \in \boldsymbol{K}_{\boldsymbol{m}}$, e.g. $\boldsymbol{U}_{\sigma}^{0} \equiv \boldsymbol{Q}_{2}^{h} \boldsymbol{u}^{0}$.

Below we recall some well-known results concerning $S^{h}$ :

$$
\begin{aligned}
& |\chi|_{m, p_{2}} \leq C h^{\frac{d\left(p_{1}-p_{2}\right)}{p_{1} p_{2}}}|\chi|_{m, p_{1}} \quad \forall \chi \in S^{h}, \quad 1 \leq p_{1} \leq p_{2} \leq \infty, \quad m=0 \text { or } 1 ; \\
& |\chi|_{1, p} \leq C h^{-1}|\chi|_{0, p} \quad \forall \chi \in S^{h}, \quad 1 \leq p \leq \infty ; \\
& \lim _{h \rightarrow 0}\left\|\left(I-\pi^{h}\right) \eta\right\|_{0, \infty}=0 \quad \forall \eta \in C(\bar{\Omega}) . \\
& \left|\left(I-Q_{1}^{h}\right) \eta\right|_{0}+h\left|\left(I-Q_{1}^{h}\right) \eta\right|_{1} \leq C h^{m}|\eta|_{m} \quad \forall \eta \in H^{m}(\Omega), \quad m=1 \text { or } 2 ; \\
& |\chi|_{0}^{2} \leq|\chi|_{h}^{2} \leq(d+2)|\chi|_{0}^{2} \quad \forall \chi \in S^{h} ; \\
& \left|\left(v^{h}, \chi\right)^{h}-\left(v^{h}, \chi\right)\right| \leq C h^{1+m}\left\|v^{h}\right\|_{m}\|\chi\|_{1} \quad \forall v^{h}, \chi \in S^{h}, \quad m=0 \text { or } 1 ;
\end{aligned}
$$

and if $d=1$

$$
\begin{gathered}
\left|\left(I-\pi^{h}\right) \eta\right|_{m, r} \leq C h^{1-m}|\eta|_{1, r} \quad \forall \eta \in W^{1, r}(\Omega), \quad m=0 \text { or } 1, \text { any } r \in[1, \infty] ; \\
\lim _{h \rightarrow 0}\left\|\left(I-\pi^{h}\right) \eta\right\|_{1}=0 \quad \forall \eta \in H^{1}(\Omega) .
\end{gathered}
$$

If $d=1$, then a simple consequence of (2.16) and (2.17) is that

$$
\begin{aligned}
\left|(v, \eta)^{h}-(v, \eta)\right| & \leq\left|\left(\pi^{h} v, \pi^{h} \eta\right)^{h}-\left(\pi^{h} v, \pi^{h} \eta\right)\right|+\left|\left(\left(I-\pi^{h}\right) v, \pi^{h} \eta\right)\right|+\left|\left(v,\left(I-\pi^{h}\right) \eta\right)\right| \\
& \leq C\left[\left|\left(I-\pi^{h}\right) v\right|_{0}+h|v|_{0}\right]\|\eta\|_{1} \quad \forall v \in C(\bar{\Omega}), \quad \forall \eta \in H^{1}(\Omega) .
\end{aligned}
$$

Comparing $Q_{2}^{h} \eta$ with $Q_{1}^{h} \eta$ and noting (2.16), (2.12) and (2.14) yields that

$$
\left|\left(I-Q_{2}^{h}\right) \eta\right|_{0}+h\left|\left(I-Q_{2}^{h}\right) \eta\right|_{1} \leq C h|\eta|_{1} \quad \forall \eta \in H^{1}(\Omega) .
$$

It follows from (2.2) that

$$
\left(Q_{2}^{h} \eta\right)\left(\boldsymbol{x}_{j}\right) \equiv \frac{\left(\eta, \beta_{j}\right)}{\left(1, \beta_{j}\right)} \quad \forall j \in J \Longrightarrow\left\|Q_{2}^{h} \eta\right\|_{0, \infty} \leq\|\eta\|_{0, \infty} \quad \forall \eta \in L^{\infty}(\Omega) .
$$

Similarly to (1.17), we introduce the operator $\hat{\mathcal{G}}^{h}: \mathcal{F}^{h} \rightarrow V^{h}$ such that

$$
\left(\nabla \hat{\mathcal{G}}^{h} v, \nabla \chi\right)=(v, \chi)^{h} \quad \forall \chi \in S^{h},
$$

where

$$
V^{h}:=\left\{v^{h} \in S^{h}:\left(v^{h}, 1\right)=0\right\} \quad \text { and } \quad \mathcal{F}^{h}:=\left\{v \in C(\bar{\Omega}):(v, 1)^{h}=0\right\}
$$


Then $\hat{\mathcal{G}}^{h}: \mathcal{F}^{h} \rightarrow \boldsymbol{V}^{h}$ is defined by $\left\{\hat{\mathcal{G}}^{h} \boldsymbol{v}\right\}_{n}:=\hat{\mathcal{G}}^{h} v_{n}$, where

$$
\begin{aligned}
& \boldsymbol{V}^{h}:=\left\{\boldsymbol{v}^{h}: v_{n}^{h} \in V^{h}, n=1 \rightarrow N, \text { and } \boldsymbol{v}^{h}\left(\boldsymbol{x}_{j}\right) \in \mathcal{M}(0), \forall j \in J\right\}, \\
& \mathcal{F}^{h}:=\left\{\boldsymbol{v}: v_{n} \in \mathcal{F}^{h}, n=1 \rightarrow N, \text { and } \boldsymbol{v}\left(\boldsymbol{x}_{j}\right) \in \mathcal{M}(0), \forall j \in J\right\} \supset \boldsymbol{V}^{h} .
\end{aligned}
$$

It is easily deduced from (1.19) and the above that under the assumptions (A)

$$
C_{1}\left\|\boldsymbol{v}^{h}\right\|_{-1} \leq\left|\hat{\mathcal{G}}^{h} \boldsymbol{v}^{h}\right|_{1} \leq C_{2}\left\|\boldsymbol{v}^{h}\right\|_{-1} \quad \forall \boldsymbol{v}^{h} \in \boldsymbol{V}^{h},
$$

see for example Barrett and Blowey [2]. We introduce the following anisotropic version of $\hat{\mathcal{G}}^{h}:$ given $\sigma \in \mathbb{R}^{+}$ and $\boldsymbol{q}^{h} \in \boldsymbol{K}_{\boldsymbol{m}}^{h}, \hat{\mathcal{G}}_{\sigma, \boldsymbol{q}^{h}}^{h}: \mathcal{F}^{h} \rightarrow \boldsymbol{V}^{h}$ is defined by

$$
\left(L^{\sigma}\left(\boldsymbol{q}^{h}\right) \nabla \hat{\mathcal{G}}_{\sigma, \boldsymbol{q}^{h}}^{h} \boldsymbol{v}, \nabla \boldsymbol{\chi}\right)=(\boldsymbol{v}, \boldsymbol{\chi})^{h} \quad \forall \boldsymbol{\chi} \in \boldsymbol{S}^{h} .
$$

On noting (2.15), (1.18) and (2.7) we deduce the well-posedness of $\hat{\mathcal{G}}^{h}$ and of $\hat{\mathcal{G}}_{\sigma, \boldsymbol{q}^{h}}^{h}$. We note for future reference that $(2.22)$ yields

$$
(\boldsymbol{v}, \boldsymbol{\chi})^{h} \equiv\left(\nabla \hat{\mathcal{G}}^{h} \boldsymbol{v}, \nabla \boldsymbol{\chi}\right) \leq\left|\hat{\mathcal{G}}^{h} \boldsymbol{v}\right|_{1}|\chi|_{1} \quad \forall \boldsymbol{v} \in \mathcal{F}^{h}, \boldsymbol{\chi} \in \boldsymbol{S}^{h} .
$$

Choosing $\chi \equiv \boldsymbol{e}^{n}, n=1 \rightarrow N$, and $\boldsymbol{\chi} \equiv \chi \mathbf{1}, \forall \chi \in S^{h}$, in (2.10a) and noting (2.5a) yields that $\boldsymbol{U}_{\sigma}^{k} \in \boldsymbol{K}_{\boldsymbol{m}}^{h}$, $k=1 \rightarrow K$. Hence it follows from (2.10a), (2.26), (2.24a,b), (2.5a), (2.7) and (1.18) that

$$
\boldsymbol{W}_{\sigma}^{k} \equiv-\hat{\mathcal{G}}_{\sigma, U_{\sigma}^{k-1}}^{h}\left(\frac{\boldsymbol{U}_{\sigma}^{k}-U_{\sigma}^{k-1}}{\tau_{k}}\right)+\Xi_{\sigma}^{k} \mathbf{1}+\boldsymbol{\Lambda}_{\sigma}^{k}
$$

where $\Xi_{\sigma}^{k} \in S^{h}$ and $\boldsymbol{\Lambda}_{\sigma}^{k} \in \mathcal{M}(0)$. Hence $\left(\mathrm{P}_{\sigma}^{h, \tau}\right)$ can be rewritten as:

Find $\left\{\boldsymbol{U}_{\sigma}^{k}, \Xi_{\sigma}^{k}, \boldsymbol{\Lambda}_{\sigma}^{k}\right\} \in \boldsymbol{K}_{\boldsymbol{m}}^{h} \times S^{h} \times \mathcal{M}(0)$ such that

$$
\begin{gathered}
\gamma\left(\nabla \boldsymbol{U}_{\sigma}^{k}, \nabla\left(\boldsymbol{\chi}-\boldsymbol{U}_{\sigma}^{k}\right)\right)+\left(\boldsymbol{D} \Psi_{1}\left(\boldsymbol{U}_{\sigma}^{k}\right)-A^{-} \boldsymbol{U}_{\sigma}^{k}+\hat{\mathcal{G}}_{\sigma, \boldsymbol{U}_{\sigma}^{k-1}}^{h}\left(\frac{\boldsymbol{U}_{\sigma}^{k}-\boldsymbol{U}_{\sigma}^{k-1}}{\tau_{k}}\right), \boldsymbol{\chi}-\boldsymbol{U}_{\sigma}^{k}\right)^{h} \\
\geq\left(\Xi_{\sigma}^{k} \mathbf{1}+\boldsymbol{\Lambda}_{\sigma}^{k}+A^{+} \boldsymbol{U}_{\sigma}^{k-1}, \boldsymbol{\chi}-\boldsymbol{U}_{\sigma}^{k}\right)^{h} \quad \forall \boldsymbol{\chi} \in \boldsymbol{K}^{h} .
\end{gathered}
$$

Theorem 2.1. Let $\Omega$ and $\mathcal{T}^{h}$ be such that assumption (A) holds and let $\boldsymbol{U}_{\sigma}^{0} \in \boldsymbol{K}_{\boldsymbol{m}}^{h}$. In addition let $L^{\sigma}$ satisfy (2.4a,b) and $\psi_{1} \in C^{1}([0,1])$ be convex. Then for all $h>0$ and time partitions $\left\{\tau_{k}\right\}_{k=1}^{K}$ there exists a solution $\left\{\boldsymbol{U}_{\sigma}^{k}, \boldsymbol{W}_{\sigma}^{k}\right\}_{k=1}^{K}$ to $\left(P_{\sigma}^{h, \tau}\right)$. Moreover, $\left\{\boldsymbol{U}_{\sigma}^{k}\right\}_{k=1}^{K}$ is unique and the following stability bounds hold

$$
\begin{aligned}
\max _{k=1 \rightarrow K}\left\|\boldsymbol{U}_{\sigma}^{k}\right\|_{1}^{2}+\sum_{k=1}^{K} \tau_{k}^{2}\left|\frac{\boldsymbol{U}_{\sigma}^{k}-U_{\sigma}^{k-1}}{\tau_{k}}\right|_{1}^{2}+\sum_{k=1}^{K} \tau_{k}\left|\left[L^{\sigma}\left(\boldsymbol{U}_{\sigma}^{k-1}\right)\right]^{\frac{1}{2}} \nabla \boldsymbol{W}_{\sigma}^{k}\right|_{0}^{2} & \\
& +\left[L_{\max }^{\sigma}\right]^{-1} \sum_{k=1}^{K} \tau_{k}\left|\hat{\mathcal{G}}^{h}\left(\frac{\boldsymbol{U}_{\sigma}^{k}-\boldsymbol{U}_{\sigma}^{k-1}}{\tau_{k}}\right)\right|_{1}^{2} \leq C\left[\left|\boldsymbol{U}_{\sigma}^{0}\right|_{1}^{2}+1\right] .
\end{aligned}
$$

Furthermore if $U_{\sigma, n}^{k}\left(\boldsymbol{x}_{j}\right)>0$, then $W_{\sigma, n}^{k}\left(\boldsymbol{x}_{j}\right)$ is unique.

Proof. We note that (2.29) with $\chi \in \boldsymbol{K}_{\boldsymbol{m}}^{h}$ is the Euler-Lagrange variational inequality of the minimization problem

$$
\begin{aligned}
\min _{\boldsymbol{v}^{h} \in \boldsymbol{K}_{\boldsymbol{m}}^{h}} \mathcal{E}^{h}\left(\boldsymbol{v}^{h}\right):= & \left\{\gamma\left|\boldsymbol{v}^{h}\right|_{1}^{2}+\frac{1}{\tau_{k}}\left|\left[L^{\sigma}\left(\boldsymbol{U}_{\sigma}^{k-1}\right)\right]^{\frac{1}{2}} \nabla \hat{\mathcal{G}}_{\sigma, U_{\sigma}^{h-1}}^{h}\left(\boldsymbol{v}^{h}-\boldsymbol{U}_{\sigma}^{k-1}\right)\right|_{0}^{2}\right. \\
& \left.+2\left(\Psi_{1}\left(\boldsymbol{v}^{h}\right), 1\right)^{h}-\left(A^{-} \boldsymbol{v}^{h}, \boldsymbol{v}^{h}\right)^{h}-2\left(A^{+} \boldsymbol{U}_{\sigma}^{k-1}, \boldsymbol{v}^{h}\right)^{h}\right\} .
\end{aligned}
$$


As $\mathcal{E}^{h}(\cdot)$ is strictly convex on $\boldsymbol{K}_{\boldsymbol{m}}^{h}$, which is convex and non-empty, $\mathcal{E}^{h}(\cdot)$ has a unique minimum $\boldsymbol{U}_{\sigma}^{k} \in \boldsymbol{K}_{\boldsymbol{m}}^{h}$. Moreover, $\boldsymbol{U}_{\sigma}^{k}$ is the unique solution of (2.29) with $\boldsymbol{\chi} \in \boldsymbol{K}_{m}^{h}$. Existence of the Lagrange multipliers $\Xi_{\sigma}^{k} \in S^{h}$ and $\Lambda_{\sigma}^{k} \in \mathcal{M}(0)$, for fixed $k$, in (2.29) then follow from standard optimisation theory, see e.g. Ciarlet [14, Th. 9.2-3]. Therefore on noting (2.28), we have existence of a solution $\left\{\boldsymbol{U}_{\sigma}^{k}, \boldsymbol{W}_{\sigma}^{k}\right\}_{k=1}^{K}$ to $\left(\mathrm{P}_{\sigma}^{h, \tau}\right)$. We cannot guarantee the uniqueness of the Lagrange multipliers $\Xi_{\sigma}^{k}, \boldsymbol{\Lambda}_{\sigma}^{k}$ and hence also of $\boldsymbol{W}_{\sigma}^{k}$. However if $U_{\sigma, n}^{k}\left(\boldsymbol{x}_{j}\right)>0$, then choosing $\boldsymbol{\chi} \equiv \boldsymbol{U}_{\sigma}^{k} \pm \frac{1}{2} U_{\sigma, n}^{k}\left(\boldsymbol{x}_{j}\right) \beta_{j} \boldsymbol{e}^{n}$ in (2.10b) yields the desired uniqueness result for the $W_{\sigma, n}^{k}\left(\boldsymbol{x}_{j}\right)$.

We now prove the stability bound (2.30). For fixed $k \geq 1$ choosing $\boldsymbol{\chi} \equiv \boldsymbol{W}_{\sigma}^{k}$ in (2.10a), $\boldsymbol{\chi} \equiv \boldsymbol{U}_{\sigma}^{k-1}$ in (2.10b) and combining yields that

$$
\begin{aligned}
\frac{\gamma}{2}\left|\boldsymbol{U}_{\sigma}^{k}\right|_{1}^{2} & +\frac{\gamma}{2}\left|\boldsymbol{U}_{\sigma}^{k}-\boldsymbol{U}_{\sigma}^{k-1}\right|_{1}^{2}-\frac{\gamma}{2}\left|\boldsymbol{U}_{\sigma}^{k-1}\right|_{1}^{2}+\left(\Psi\left(\boldsymbol{U}_{\sigma}^{k}\right), 1\right)^{h}-\left(\Psi\left(\boldsymbol{U}_{\sigma}^{k-1}\right), 1\right)^{h} \\
& +\frac{1}{2}\left(\left(A^{+}-A^{-}\right)\left(\boldsymbol{U}_{\sigma}^{k}-\boldsymbol{U}_{\sigma}^{k-1}\right), \boldsymbol{U}_{\sigma}^{k}-\boldsymbol{U}_{\sigma}^{k-1}\right)^{h}+\tau_{k}\left|\left[L^{\sigma}\left(\boldsymbol{U}_{\sigma}^{k-1}\right)\right]^{\frac{1}{2}} \nabla \boldsymbol{W}_{\sigma}^{k}\right|_{0}^{2} \\
\leq & \gamma\left(\nabla \boldsymbol{U}_{\sigma}^{k}, \nabla\left(\boldsymbol{U}_{\sigma}^{k}-\boldsymbol{U}_{\sigma}^{k-1}\right)\right)+\tau_{k}\left|\left[L^{\sigma}\left(\boldsymbol{U}_{\sigma}^{k-1}\right)\right]^{\frac{1}{2}} \nabla \boldsymbol{W}_{\sigma}^{k}\right|_{0}^{2} \\
& +\left(\boldsymbol{D} \psi_{1}\left(\boldsymbol{U}_{\sigma}^{k}\right)-A^{-} \boldsymbol{U}_{\sigma}^{k}-A^{+} \boldsymbol{U}_{\sigma}^{k-1}, \boldsymbol{U}_{\sigma}^{k}-\boldsymbol{U}_{\sigma}^{k-1}\right)^{h} \leq 0
\end{aligned}
$$

where we have noted the convexity of $\psi_{1},(2.3)$ and the following identity for symmetric $N \times N$ matrices $B$

$$
-2(\boldsymbol{\zeta}-\boldsymbol{\eta})^{T} B \boldsymbol{\eta} \equiv \boldsymbol{\eta}^{T} B \boldsymbol{\eta}-\boldsymbol{\zeta}^{T} B \boldsymbol{\zeta}+(\boldsymbol{\zeta}-\boldsymbol{\eta})^{T} B(\boldsymbol{\zeta}-\boldsymbol{\eta}) \quad \forall \boldsymbol{\zeta}, \boldsymbol{\eta} \in \mathbb{R}^{N}
$$

Summing (2.32) from $k=1 \rightarrow m$, for $m=1 \rightarrow K$, and noting the properties of $\Psi$, (1.18) and $f \boldsymbol{U}_{\sigma}^{k}=f \boldsymbol{u}^{0}$ yields the first three bounds in (2.30). Choosing $\boldsymbol{\chi} \equiv \hat{\mathcal{G}}^{h}\left(\frac{\boldsymbol{U}_{\sigma}^{k}-\boldsymbol{U}_{\sigma}^{k-1}}{\tau_{k}}\right)$ in (2.10a) and noting (2.22) and (2.9) yields for $k \geq 1$ that

$$
\begin{aligned}
\left|\hat{\mathcal{G}}^{h}\left(\frac{\boldsymbol{U}_{\sigma}^{k}-\boldsymbol{U}_{\sigma}^{k-1}}{\tau_{k}}\right)\right|_{1}^{2} & =\left(\frac{\boldsymbol{U}_{\sigma}^{k}-\boldsymbol{U}_{\sigma}^{k-1}}{\tau_{k}}, \hat{\mathcal{G}}^{h}\left(\frac{\boldsymbol{U}_{\sigma}^{k}-U_{\sigma}^{k-1}}{\tau_{k}}\right)\right)^{h} \\
& =-\left(L^{\sigma}\left(\boldsymbol{U}_{\sigma}^{k-1}\right) \nabla \boldsymbol{W}_{\sigma}^{k}, \nabla \hat{\mathcal{G}}^{h}\left(\frac{\boldsymbol{U}_{\sigma}^{k}-U_{\sigma}^{k-1}}{\tau_{k}}\right)\right) \\
& \leq\left|\left[L^{\sigma}\left(\boldsymbol{U}_{\sigma}^{k-1}\right)\right] \nabla \boldsymbol{W}_{\sigma}^{k}\right|_{0}^{2} \leq L_{\max }^{\sigma}\left|\left[L^{\sigma}\left(\boldsymbol{U}_{\sigma}^{k-1}\right)\right]^{\frac{1}{2}} \nabla \boldsymbol{W}_{\sigma}^{k}\right|_{0}^{2} .
\end{aligned}
$$

Summing (2.34) from $k=1 \rightarrow K$ and noting the third bound in (2.30) yields the desired fourth bound in $(2.30)$.

Remark 2.1. As can be seen from $(2.32), \frac{\gamma}{2}|\cdot|_{1}^{2}+(\Psi(\cdot), 1)^{h}$ has the property that it is a Lyapunov functional for the discrete evolution of the approximation $\left(\mathrm{P}_{\sigma}^{h, \tau}\right)$.

Instead of $\left(\mathrm{P}_{\sigma}^{h, \tau}\right)$ one could consider the corresponding non-regularised approximation of $(\mathrm{P})$ : $\left(\mathbf{P}^{h, \tau}\right)$ For $k \geq 1$, find $\left\{\boldsymbol{U}^{k}, \boldsymbol{W}^{k}\right\} \in \boldsymbol{K}^{h} \times \boldsymbol{S}^{h}$ such that

$$
\begin{aligned}
& \left(\frac{\boldsymbol{U}^{k}-\boldsymbol{U}^{k-1}}{\tau_{k}}, \boldsymbol{\chi}\right)^{h}+\left(L\left(\boldsymbol{U}^{k-1}\right) \nabla \boldsymbol{W}^{k}, \nabla \boldsymbol{\chi}\right)=0 \quad \forall \boldsymbol{\chi} \in \boldsymbol{S}^{h}, \\
& \gamma\left(\nabla \boldsymbol{U}^{k}, \nabla\left(\boldsymbol{\chi}-\boldsymbol{U}^{k}\right)\right)+\left(\boldsymbol{D} \Psi_{1}\left(\boldsymbol{U}^{k}\right)-A^{-} \boldsymbol{U}^{k}-\boldsymbol{W}^{k}, \boldsymbol{\chi}-\boldsymbol{U}^{k}\right)^{h} \geq\left(A^{+} \boldsymbol{U}^{k-1}, \boldsymbol{\chi}-\boldsymbol{U}^{k}\right)^{h} \quad \forall \boldsymbol{\chi} \in \boldsymbol{K}^{h} ;
\end{aligned}
$$

where $\boldsymbol{U}^{0} \in \boldsymbol{K}_{\boldsymbol{m}}^{h}$ is an approximation of $\boldsymbol{u}^{0} \in \boldsymbol{K}_{\boldsymbol{m}}$, e.g. $\boldsymbol{U}^{0} \equiv \boldsymbol{Q}_{2}^{h} \boldsymbol{u}^{0}$. Unfortunately, we are not able to prove existence of a solution $\left\{\boldsymbol{U}^{k}, \boldsymbol{W}^{k}\right\}_{k=1}^{K}$ to $\left(\mathrm{P}^{h, \tau}\right)$. Given $\boldsymbol{U}^{k-1}$; then one approach is to replace $L$ by $L^{\sigma}$ in (2.35a) and demonstrate the existence of a solution $\left\{\boldsymbol{U}^{k, \sigma}, \boldsymbol{W}^{k, \sigma}\right\}$, which is bounded independently of $\sigma$, as for $\left(\mathrm{P}_{\sigma}^{h, \tau}\right)$. However, we are not able to show that the $\sigma^{\prime} \rightarrow 0$ limit of a convergent subsequence $\left\{\boldsymbol{U}^{k, \sigma^{\prime}}, \boldsymbol{W}^{k, \sigma^{\prime}}\right\}_{\sigma^{\prime}>0}$ solves $\left(\mathrm{P}^{h, \tau}\right)$. 
An alternative approach is to consider the well-posedness of the non-regularised version of (2.26). To do this we try to extend the argument for the scalar Cahn-Hilliard equation with degenerate mobility in Barrett et al. [8] to the present multi-component case. Firstly, we introduce some notation. Given $\boldsymbol{q}^{h} \in \boldsymbol{K}_{\boldsymbol{m}}^{h}$, we define for each component $q_{n}^{h}, n=1 \rightarrow N$, a set of passive nodes $J_{0}\left(q_{n}^{h}\right) \subset J$ by

$$
j \in J_{0}\left(q_{n}^{h}\right) \Longleftrightarrow\left(L_{n n}\left(\boldsymbol{q}^{h}\right), \beta_{j}\right)=0 \Longleftrightarrow q_{n}^{h} \equiv 0 \text { or } 1 \text { on } \operatorname{supp}\left(\beta_{j}\right)
$$

where the second equivalence above follows immediately from (1.6). All other nodes we call active nodes for $q_{n}^{h}$ and they can be uniquely partitioned so that $J_{+}\left(q_{n}^{h}\right):=J \backslash J_{0}\left(q_{n}^{h}\right) \equiv \bigcup_{m=1}^{M_{n}} I_{m}\left(q_{n}^{h}\right), M_{n} \geq 1$; where $I_{m}\left(q_{n}^{h}\right)$, $m=1 \rightarrow M_{n}$, are mutually disjoint and maximally connected in the following sense: $I_{m}\left(q_{n}^{h}\right)$ is said to be connected if for all $j, k \in I_{m}\left(q_{n}^{h}\right)$, there exist $\left\{\kappa_{\ell}\right\}_{\ell=1}^{\mathcal{L}} \subseteq \mathcal{T}^{h}$, not necessarily distinct, such that

$$
\begin{array}{lll}
\text { (a) } \boldsymbol{x}_{j} \in \bar{\kappa}_{1}, \quad \boldsymbol{x}_{k} \in \bar{\kappa}_{\mathcal{L}}, & \\
\text { (b) } \bar{\kappa}_{\ell} \cap \bar{\kappa}_{\ell+1} \neq \emptyset & \ell=1 \rightarrow \mathcal{L}-1, \\
\text { (c) } & L_{n n}\left(\boldsymbol{q}^{h}\right) \neq \equiv 0 \text { on } \kappa_{\ell} & \ell=1 \rightarrow \mathcal{L} .
\end{array}
$$

$I_{m}\left(q_{n}^{h}\right)$ is said to be maximally connected if there is no other connected subset of $J_{+}\left(q_{n}^{h}\right)$, which contains $I_{m}\left(q_{n}^{h}\right)$. Clearly $J_{+}\left(q_{n}^{h}\right)$ is non-empty, since if $\left(L_{n n}\left(\boldsymbol{q}^{h}\right), \beta_{j}\right)=0 \forall j \in J$ then $L_{n n}\left(\boldsymbol{q}^{h}\right) \equiv 0$ a.e. and since $\boldsymbol{q}^{h} \in \boldsymbol{K}_{\boldsymbol{m}}^{h}$ it follows that $q_{n}^{h} \equiv 0$ or 1 and hence either $\{\boldsymbol{m}\}_{n} \equiv f q_{n}^{h}=0$ or 1 , so one or more components of $\boldsymbol{u}$ are not present, in which case the system can be modelled with a smaller value of $N$.

In addition we note that if $j \in I_{m_{1}}\left(q_{n_{1}}^{h}\right)$, then $j \in I_{m_{2}}\left(q_{n_{2}}^{h}\right)$ for some $n_{2} \neq n_{1}$ and some $m_{2}$. We then set

$$
V^{h}\left(q_{n}^{h}\right):=\left\{v^{h} \in S^{h}: v^{h}\left(\boldsymbol{x}_{j}\right)=0 \quad \forall j \in J_{0}\left(q_{n}^{h}\right) \text { and }\left(v^{h}, \Delta_{m}\left(q_{n}^{h}\right)\right)^{h}=0, m=1 \rightarrow M_{n}\right\},
$$

where for $m=1 \rightarrow M_{n}$

$$
\Delta_{m}\left(q_{n}^{h}\right):=\sum_{j \in I_{m}\left(q_{n}^{h}\right)} \beta_{j}
$$

For later use, we set

$$
\Omega_{m}\left(q_{n}^{h}\right):=\left\{\bigcup_{\kappa \in \mathcal{T}^{h}} \bar{\kappa}: \Delta_{m}\left(q_{n}^{h}\right)(\boldsymbol{x})=1 \text { for all } \boldsymbol{x} \in \kappa\right\}
$$

An immediate consequence of the above definitions is that on any $\kappa \in \mathcal{T}^{h}$ and for any $n$

$$
\begin{array}{rc}
\text { either } & L_{n j}\left(\boldsymbol{q}^{h}\right) \equiv 0 \quad j=1 \rightarrow N \\
\text { or } & \Delta_{m_{\star}}\left(q_{n}^{h}\right) \equiv 1 \text { for some } m_{\star} \text { and } \Delta_{m}\left(q_{n}^{h}\right) \equiv 0 \text { for } m \neq m_{\star} .
\end{array}
$$

Finally, we introduce

$$
\begin{aligned}
& \boldsymbol{V}^{h}\left(\boldsymbol{q}^{h}\right):=V^{h}\left(q_{1}^{h}\right) \times V^{h}\left(q_{2}^{h}\right) \times \cdots \times V^{h}\left(q_{n}^{h}\right) \\
& \hat{\boldsymbol{V}}^{h}\left(\boldsymbol{q}^{h}\right):=\left\{\boldsymbol{v}^{h} \in \boldsymbol{V}^{h}\left(\boldsymbol{q}^{h}\right): \boldsymbol{v}^{h}\left(\boldsymbol{x}_{j}\right) \in \mathcal{M}(0) \quad \forall j \in J\right\} .
\end{aligned}
$$

We are now ready to introduce the following degenerate version of (2.26): given $\boldsymbol{q}^{h} \in \boldsymbol{K}_{\boldsymbol{m}}^{h}, \hat{\mathcal{G}}_{\boldsymbol{q}^{h}}^{h}: \hat{\boldsymbol{V}}^{h}\left(\boldsymbol{q}^{h}\right) \rightarrow$ $\hat{\boldsymbol{V}}^{h}\left(\boldsymbol{q}^{h}\right)$ is defined by

$$
\left(L\left(\boldsymbol{q}^{h}\right) \nabla \hat{\mathcal{G}}_{\boldsymbol{q}^{h}}^{h} \boldsymbol{v}^{h}, \nabla \boldsymbol{\chi}\right)=\left(\boldsymbol{v}^{h}, \boldsymbol{\chi}\right)^{h} \quad \forall \boldsymbol{\chi} \in \boldsymbol{S}^{h}
$$


In order to show the well-posedness of $\hat{\mathcal{G}}_{q^{h}}^{h}$ we first note that on choosing $\chi \equiv \beta_{j} \boldsymbol{e}^{n}$, for any $j \in J_{0}\left(q_{n}^{h}\right)$, in (2.43) leads to both sides vanishing on noting (2.36), (2.42a,b) and (2.38). Secondly, choosing $\boldsymbol{\chi} \equiv \Delta_{m}\left(q_{n}^{h}\right) \boldsymbol{e}^{n}$, $m=1 \rightarrow M_{n}, n=1 \rightarrow N$, in (2.43) leads to both sides vanishing on noting (2.41), (2.42a,b) and (2.38). Thirdly, choosing $\chi \equiv \beta_{j} \mathbf{1}, \forall j \in J$, in (2.43) leads to both sides vanishing on noting (1.14a) and (2.42b). Therefore for the well-posedness of $\hat{\mathcal{G}}_{\boldsymbol{q}^{h}}^{h}$, it remains to prove uniqueness, as $\hat{\boldsymbol{V}}^{h}\left(\boldsymbol{q}^{h}\right)$ has finite dimension. If there exist two solutions $\boldsymbol{Z}^{i} \in \hat{\boldsymbol{V}}^{h}\left(\boldsymbol{q}^{h}\right), i=1,2$, to (2.43) then $\boldsymbol{Z}^{d}:=\boldsymbol{Z}^{1}-\boldsymbol{Z}^{2} \in \hat{\boldsymbol{V}}^{h}\left(\boldsymbol{q}^{h}\right)$ satisfies on noting (1.14b) and (1.13)

$$
\int_{\Omega} q_{n}^{h} q_{p}^{h}\left|\nabla\left(Z_{n}^{d}-Z_{p}^{d}\right)\right|^{2} \mathrm{~d} \boldsymbol{x}=0 \quad n, p=1 \rightarrow N .
$$

Hence it follows from (2.44), (2.41), (2.39) and (2.40) that $\boldsymbol{Z}^{d} \in \hat{\boldsymbol{V}}^{h}\left(\boldsymbol{q}^{h}\right)$ is such that

$$
\begin{aligned}
& Z_{n_{1}}^{d}-Z_{n_{2}}^{d} \text { is constant on } \Omega_{m_{1}}\left(q_{n_{1}}^{h}\right) \cap \Omega_{m_{2}}\left(q_{n_{2}}^{h}\right) \\
& \qquad m_{1}=1 \rightarrow M_{n_{1}}, \quad m_{2}=1 \rightarrow M_{n_{2}} ; \quad n_{1}, n_{2}=1 \rightarrow N .
\end{aligned}
$$

Unfortunately, we are not able to conclude from (2.45) that $\boldsymbol{Z}^{d} \equiv \mathbf{0}$ and hence the well-posedness of $\hat{\mathcal{G}}_{q^{h}}^{h}$. If we could establish the well-posedness of $\hat{\mathcal{G}}_{\boldsymbol{q}^{h}}^{h}$, then it follows from (2.35a) and (2.43) that for $k \geq 1$, given $\boldsymbol{U}^{k-1} \in \boldsymbol{K}_{\boldsymbol{m}}^{h}$, we seek $\boldsymbol{U}^{k} \in \boldsymbol{K}^{h}\left(\boldsymbol{U}^{k-1}\right)$, where

$$
\boldsymbol{K}^{h}\left(\boldsymbol{U}^{k-1}\right):=\left\{\boldsymbol{\chi} \in \boldsymbol{K}^{h}: \boldsymbol{\chi}-\boldsymbol{U}^{k-1} \in \hat{\boldsymbol{V}}^{h}\left(\boldsymbol{U}^{k-1}\right)\right\} \subset \boldsymbol{K}_{\boldsymbol{m}}^{h} .
$$

On introducing

$$
\begin{aligned}
Y_{n}^{h} & :=\operatorname{span}\left\{\left\{\Delta_{m}\left(q_{n}^{h}\right)\right\}_{m=1}^{M_{n}},\left\{\beta_{j}\right\}_{j \in J_{0}^{h}\left(q_{n}^{h}\right)}\right\}, \\
\boldsymbol{Y}^{h} & :=Y_{1}^{h} \times Y_{2}^{h} \times \cdots Y_{N}^{h}, \\
\hat{\boldsymbol{Y}}^{h} & :=\left\{\boldsymbol{y}^{h} \in \boldsymbol{Y}^{h}: \boldsymbol{y}^{h}\left(\boldsymbol{x}_{j}\right) \in \mathcal{M}(0) \quad \forall j \in J\right\}
\end{aligned}
$$

then a solution $\boldsymbol{W}^{k}$ in $(2.35 \mathrm{a}, \mathrm{b})$ could be expressed in terms of $\boldsymbol{U}^{k}$ as (cf. (2.43) and (2.42a,b))

$$
\boldsymbol{W}^{k} \equiv-\hat{\mathcal{G}}_{\boldsymbol{U}^{k-1}}^{h}\left(\frac{\boldsymbol{U}^{k}-\boldsymbol{U}^{k-1}}{\tau_{k}}\right)+\Xi^{k} \mathbf{1}+\boldsymbol{\Lambda}^{k}
$$

where $\Xi^{k} \in S^{h}$ and $\boldsymbol{\Lambda}^{k} \in \hat{\boldsymbol{Y}}^{h}$. Hence $\left(\mathrm{P}^{h, \tau}\right)$ could then be restated as:

For $k \geq 1$, find $\left\{\boldsymbol{U}^{k}, \Xi^{k}, \boldsymbol{\Lambda}^{k}\right\} \in \boldsymbol{K}^{h}\left(\boldsymbol{U}^{k-1}\right) \times S^{h} \times \hat{\boldsymbol{Y}}^{h}$ such that

$$
\begin{gathered}
\gamma\left(\nabla \boldsymbol{U}^{k}, \nabla\left(\boldsymbol{\chi}-\boldsymbol{U}^{k}\right)\right)+\left(\boldsymbol{D} \Psi_{1}\left(\boldsymbol{U}^{k}\right)-A^{-} \boldsymbol{U}^{k}+\hat{\mathcal{G}}_{\boldsymbol{U}^{k-1}}^{h}\left(\frac{\boldsymbol{U}^{k}-\boldsymbol{U}^{k-1}}{\tau_{k}}\right), \boldsymbol{\chi}-\boldsymbol{U}^{k}\right)^{h} \\
\geq\left(\Xi^{k} \mathbf{1}+\boldsymbol{\Lambda}^{k}+A^{+} \boldsymbol{U}^{k-1}, \boldsymbol{\chi}-\boldsymbol{U}^{k}\right)^{h} \quad \forall \boldsymbol{\chi} \in \boldsymbol{K}^{h} .
\end{gathered}
$$

By considering the associated minimization problem, (2.31) with $\boldsymbol{K}_{\boldsymbol{m}}^{h}$ replaced by $\boldsymbol{K}^{h}\left(\boldsymbol{U}^{k-1}\right)$ and the $\sigma$ superscripts/subscripts removed, the existence of a solution $\left\{\boldsymbol{U}^{k}, \Xi^{k}, \boldsymbol{\Lambda}^{k}\right\}$ to (2.49) is then easily established and hence, on noting (2.48), existence of a solution $\left\{\boldsymbol{U}^{k}, \boldsymbol{W}^{k}\right\}$ to $\left(\mathrm{P}^{h, \tau}\right)$ at time level $t_{k}$.

Therefore if one could show that $(2.45) \Longrightarrow \boldsymbol{Z}^{d} \equiv \mathbf{0}$, then existence of a solution $\left\{\boldsymbol{U}^{k}, \boldsymbol{W}^{k}\right\}_{k=1}^{K}$ to $\left(\mathrm{P}^{h, \tau}\right)$ immediately follows. As stated above, unfortunately we have not been able to prove that $(2.45) \Longrightarrow \boldsymbol{Z}^{d} \equiv \mathbf{0}$; except for the case $N=2$ when (P) collapses to the scalar Cahn-Hilliard equation with degenerate mobility studied in Barrett et al. [8]. However, we know of no counterexamples. We note that if we assume the existence of a solution $\left\{\boldsymbol{U}^{k}, \boldsymbol{W}^{k}\right\}_{k=1}^{K}$ to $\left(\mathrm{P}^{h, \tau}\right)$; then it is a simple matter to check that the corresponding uniqueness and stability results of Theorem 2.1, the $\sigma$ superscripts and subscripts removed, hold. In practice we have not experienced any difficulty in computing with the approximation $\left(\mathrm{P}^{h, \tau}\right)$, see the numerical experiments in 
Section 5. Below we establish the convergence in one space dimension $(d=1)$ of the regularised approximation $\left(\mathrm{P}_{\sigma}^{h, \tau}\right)$ as $h, \tau$ and $\sigma \rightarrow 0$; and of the approximation $\left(\mathrm{P}^{h, \tau}\right)$, on assuming existence, as $h$ and $\tau \rightarrow 0$. Below we adopt the notation ${ }_{(\sigma)}$, which is an abbreviation for either "with" or "without" the subscript $\sigma$; similarly for superscripts.

Let

$$
\boldsymbol{U}_{(\sigma)}(t):=\frac{t-t_{k-1}}{\tau_{k}} \boldsymbol{U}_{(\sigma)}^{k}+\frac{t_{k}-t}{\tau_{k}} \boldsymbol{U}_{(\sigma)}^{k-1} \quad t \in\left[t_{k-1}, t_{k}\right] \quad k \geq 1
$$

and

$$
\boldsymbol{U}_{(\sigma)}^{+}(t):=\boldsymbol{U}_{(\sigma)}^{k}, \quad \boldsymbol{U}_{(\sigma)}^{-}(t):=\boldsymbol{U}_{(\sigma)}^{k-1} \quad t \in\left(t_{k-1}, t_{k}\right] \quad k \geq 1
$$

We note for future reference that

$$
\boldsymbol{U}_{(\sigma)}-\boldsymbol{U}_{(\sigma)}^{ \pm}=\left(t-t_{k}^{ \pm}\right) \frac{\partial}{\partial t} \boldsymbol{U}_{(\sigma)} \quad t \in\left(t_{k-1}, t_{k}\right) \quad k \geq 1,
$$

where $t_{k}^{+}:=t_{k}$ and $t_{k}^{-}:=t_{k-1}$. We introduce also

$$
\bar{\tau}(t):=\tau_{k} \quad t \in\left(t_{k-1}, t_{k}\right] \quad k \geq 1
$$

Using the above notation and introducing analogous notation for $\boldsymbol{W}_{(\sigma)} ;\left(\mathrm{P}_{(\sigma)}^{h, \tau}\right)$ can be restated as: Find $\left\{\boldsymbol{U}_{(\sigma)}, \boldsymbol{W}_{(\sigma)}\right\} \in H^{1}\left(0, T ; \boldsymbol{K}^{h}\right) \times L^{2}\left(0, T ; \boldsymbol{S}^{h}\right)$ such that

$$
\begin{gathered}
\int_{0}^{T}\left[\left(\frac{\partial}{\partial t} \boldsymbol{U}_{(\sigma)}, \boldsymbol{\chi}\right)^{h}+\left(L^{(\sigma)}\left(\boldsymbol{U}_{(\sigma)}^{-}\right) \nabla \boldsymbol{W}_{(\sigma)}^{+}, \nabla \boldsymbol{\chi}\right)\right] \mathrm{d} t=0 \quad \forall \boldsymbol{\chi} \in L^{2}\left(0, T ; \boldsymbol{S}^{h}\right) \\
\int_{0}^{T}\left[\gamma\left(\nabla \boldsymbol{U}_{(\sigma)}^{+}, \nabla\left(\boldsymbol{\chi}-\boldsymbol{U}_{(\sigma)}^{+}\right)\right)+\left(\boldsymbol{D} \Psi_{1}\left(\boldsymbol{U}_{(\sigma)}^{+}\right)-A^{-} \boldsymbol{U}_{(\sigma)}^{+}, \boldsymbol{\chi}-\boldsymbol{U}_{(\sigma)}^{+}\right)^{h}\right] \mathrm{d} t \\
\geq \int_{0}^{T}\left(A^{+} \boldsymbol{U}_{(\sigma)}^{-}+\boldsymbol{W}_{(\sigma)}^{+}, \boldsymbol{\chi}-\boldsymbol{U}_{(\sigma)}^{+}\right)^{h} \mathrm{~d} t \quad \forall \boldsymbol{\chi} \in L^{2}\left(0, T ; \boldsymbol{K}^{h}\right) .
\end{gathered}
$$

Theorem 2.2. Let $\psi_{1} \in C^{1}([0,1])$ be convex and $L^{(\sigma)}$ satisfy (1.6), (1.13) and (2.4a,b). Let $d=1$ and $\boldsymbol{u}^{0} \in \boldsymbol{K}_{\boldsymbol{m}}$. Let $\left\{\mathcal{T}^{h}, \boldsymbol{U}_{(\sigma)}^{0}, \tau(, \sigma)\right\}_{h>0}$ be such that

i) $\boldsymbol{U}_{(\sigma)}^{0} \in \boldsymbol{K}_{\boldsymbol{m}}^{h}$ and $\boldsymbol{U}_{(\sigma)}^{0} \rightarrow \boldsymbol{u}^{0}$ in $\boldsymbol{H}^{1}(\Omega)$ as $h \rightarrow 0$,

ii) $\Omega$ and $\left\{\mathcal{T}^{h}\right\}_{h>0}$ fulfill assumption $(A)$,

iii) $\tau(, \sigma) \rightarrow 0$ as $h \rightarrow 0$.

Then there exists a subsequence of $\left\{\boldsymbol{U}_{(\sigma)}, \boldsymbol{W}_{(\sigma)}\right\}_{h}$, assuming the existence of a solution in the case of $\left(P^{h, \tau}\right)$, and a vector function $\boldsymbol{u} \in L^{\infty}\left(0, T ; \boldsymbol{K}_{\boldsymbol{m}}\right) \cap H^{1}\left(0, T ;\left(\boldsymbol{H}^{1}(\Omega)\right)^{\prime}\right) \cap \boldsymbol{C}_{x, t}^{\frac{1}{2}, \frac{1}{8}}\left(\bar{\Omega}_{T}\right)$ and functions $z_{n p} \in L_{\text {loc }}^{2}\left(\left\{u_{n}>\right.\right.$ $\left.0\} \cap\left\{u_{p}>0\right\}\right), n, p=1 \rightarrow N$ with $\frac{\partial}{\partial x} z_{n p} \in L_{l o c}^{2}\left(\left\{u_{n}>0\right\} \cap\left\{u_{p}>0\right\}\right)$ such that as $h \rightarrow 0$

$$
\begin{aligned}
\boldsymbol{U}_{(\sigma)}, \boldsymbol{U}_{(\sigma)}^{ \pm} \rightarrow \boldsymbol{u} & \text { uniformly on } \bar{\Omega}_{T}, \\
\boldsymbol{U}_{(\sigma)}, \boldsymbol{U}_{(\sigma)}^{ \pm} \rightarrow \boldsymbol{u} & \text { weakly in } L^{2}\left(0, T ; \boldsymbol{H}^{1}(\Omega)\right), \\
W_{(\sigma,) n}^{+}-W_{(\sigma,) p}^{+} \rightarrow z_{n p}, \quad \frac{\partial}{\partial x}\left(W_{(\sigma,) n}^{+}-W_{(\sigma,) p}^{+}\right) \rightarrow \frac{\partial}{\partial x} z_{n p} \quad \begin{array}{l}
\text { weakly in } L_{l o c}^{2}\left(\left\{u_{n}>0\right\} \cap\left\{u_{p}>0\right\}\right), \\
n, p=1 \rightarrow N ;
\end{array} &
\end{aligned}
$$

where $\left\{u_{n}>0\right\}:=\left\{(x, t) \in \Omega_{T}: u_{n}(x, t)>0\right\}$. 
Furthermore $\boldsymbol{u}$ and $z_{n p}, n, p=1 \rightarrow N$, fulfill $\boldsymbol{u}(\cdot, 0)=\boldsymbol{u}^{0}(\cdot)$ and

$$
\begin{gathered}
\int_{0}^{T}\left\langle\frac{\partial}{\partial t} u_{n}, \eta\right\rangle \mathrm{d} t-\sum_{p=1}^{N} \int_{\left\{u_{n}>0\right\} \cap\left\{u_{p}>0\right\}} L_{n p}(\boldsymbol{u}) \frac{\partial}{\partial x} z_{n p} \frac{\partial}{\partial x} \eta \mathrm{d} x \mathrm{~d} t=0 \\
\forall \eta \in L^{2}\left(0, T ; H^{1}(\Omega)\right), \quad n=1 \rightarrow N ; \\
\int_{\left\{u_{n}>0\right\} \cap\left\{u_{p}>0\right\}}\left[\left(\left[\psi_{1}^{\prime}\left(u_{n}\right)-\psi_{1}^{\prime}\left(u_{p}\right)\right]-\left[\{A \boldsymbol{u}\}_{n}-\{A \boldsymbol{u}\}_{p}\right]-z_{n p}\right) \eta+\gamma \frac{\partial}{\partial x}\left(u_{n}-u_{p}\right) \frac{\partial}{\partial x} \eta\right] \mathrm{d} x \mathrm{~d} t=0 \\
\forall \eta \in L^{2}\left(0, T ; H^{1}(\Omega)\right) \text { with } \operatorname{supp}(\eta) \subset\left\{u_{n}>0\right\} \cap\left\{u_{p}>0\right\}, \quad n, p=1 \rightarrow N .
\end{gathered}
$$

The latter immediately implies that

$$
\begin{gathered}
z_{n p}=-\gamma \frac{\partial^{2}}{\partial x^{2}}\left(u_{n}-u_{p}\right)+\left[\psi_{1}^{\prime}\left(u_{n}\right)-\psi_{1}^{\prime}\left(u_{p}\right)\right]-\left[\{A \boldsymbol{u}\}_{n}-\{A \boldsymbol{u}\}_{p}\right] \\
\text { on the set }\left\{u_{n}>0\right\} \cap\left\{u_{p}>0\right\}, \quad n, p=1 \rightarrow N .
\end{gathered}
$$

Proof. Noting the definition (2.50a,b), (2.52), (2.30) and it's non-regularised counterpart, (2.25), assumptions i)-iii), (1.18) together with the fact that $\boldsymbol{U}_{(\sigma)}^{( \pm)} \in \boldsymbol{K}_{m}^{h}$ imply

$$
\begin{aligned}
\left\|\boldsymbol{U}_{(\sigma)}\right\|_{L^{\infty}\left(0, T ; \boldsymbol{H}^{1}(\Omega)\right)}^{2}+\left\|\bar{\tau}^{\frac{1}{2}} \frac{\partial}{\partial t} \boldsymbol{U}_{(\sigma)}\right\|_{L^{2}\left(0, T ; \boldsymbol{H}^{1}(\Omega)\right)}^{2} & \\
& +\left\|\boldsymbol{U}_{(\sigma)}\right\|_{H^{1}\left(0, T ;\left(\boldsymbol{H}^{1}(\Omega)\right)^{\prime}\right)}^{2}+\left\|\left[L^{(\sigma)}\left(\boldsymbol{U}_{(\sigma)}^{-}\right)\right]^{\frac{1}{2}} \frac{\partial}{\partial x} \boldsymbol{W}_{(\sigma)}^{+}\right\|_{\boldsymbol{L}^{2}\left(\Omega_{T}\right)}^{2} \leq C .
\end{aligned}
$$

Furthermore, we deduce from (2.51), (2.52) and (2.58) that

$$
\left\|\boldsymbol{U}_{(\sigma)}-\boldsymbol{U}_{(\sigma)}^{ \pm}\right\|_{L^{2}\left(0, T ; \boldsymbol{H}^{1}(\Omega)\right)}^{2} \leq\left\|\bar{\tau} \frac{\partial}{\partial t} \boldsymbol{U}_{(\sigma)}\right\|_{L^{2}\left(0, T ; \boldsymbol{H}^{1}(\Omega)\right)}^{2} \leq C \tau .
$$

In the next step we show that the discrete solutions $\boldsymbol{U}_{(\sigma)}$ are uniformly Hölder continuous. The first bound in (2.58) gives via a standard embedding result

$$
\left|\boldsymbol{U}_{(\sigma)}\left(y_{2}, t\right)-\boldsymbol{U}_{(\sigma)}\left(y_{1}, t\right)\right| \leq C\left|y_{2}-y_{1}\right|^{\frac{1}{2}} \quad \forall y_{1}, y_{2} \in \bar{\Omega}, \quad \forall t \geq 0 .
$$

In addition it follows from (1.16), (2.15), (2.27), (2.58) and (2.30) that

$$
\begin{aligned}
\left\|\boldsymbol{U}_{(\sigma)}\left(\cdot, t_{b}\right)-\boldsymbol{U}_{(\sigma)}\left(\cdot, t_{a}\right)\right\|_{0, \infty} & \leq C\left\|\boldsymbol{U}_{(\sigma)}\left(\cdot, t_{b}\right)-\boldsymbol{U}_{(\sigma)}\left(\cdot, t_{a}\right)\right\|_{0}^{\frac{1}{2}}\left\|\boldsymbol{U}_{(\sigma)}\left(\cdot, t_{b}\right)-\boldsymbol{U}_{(\sigma)}\left(\cdot, t_{a}\right)\right\|_{1}^{\frac{1}{2}} \\
& \leq C\left|\hat{\mathcal{G}}^{h}\left(\boldsymbol{U}_{(\sigma)}\left(\cdot, t_{b}\right)-\boldsymbol{U}_{(\sigma)}\left(\cdot, t_{a}\right)\right)\right|_{1}^{\frac{1}{4}}\left\|\boldsymbol{U}_{(\sigma)}\left(\cdot, t_{b}\right)-\boldsymbol{U}_{(\sigma)}\left(\cdot, t_{a}\right)\right\|_{1}^{\frac{3}{4}} \\
& \leq C\left|\hat{\mathcal{G}}^{h}\left[\int_{t_{a}}^{t_{b}} \frac{\partial}{\partial t} \boldsymbol{U}_{(\sigma)}(\cdot, t) \mathrm{d} t\right]\right|_{1}^{\frac{1}{4}}\left(2\left\|\boldsymbol{U}_{(\sigma)}\right\|_{L^{\infty}\left(0, T ; \boldsymbol{H}^{1}(\Omega)\right)}\right)^{\frac{3}{4}} \\
& \leq C\left|\int_{t_{a}}^{t_{b}} \hat{\mathcal{G}}^{h} \frac{\partial}{\partial t} \boldsymbol{U}_{(\sigma)}(\cdot, t) \mathrm{d} t\right|_{1}^{\frac{1}{4}} \\
& \leq C\left(t_{b}-t_{a}\right)^{\frac{1}{8}}\left(\int_{t_{a}}^{t_{b}}\left|\hat{\mathcal{G}}^{h} \frac{\partial}{\partial t} \boldsymbol{U}_{(\sigma)}\right|_{1}^{2} \mathrm{~d} t\right)^{\frac{1}{8}} \\
& \leq C\left(t_{b}-t_{a}\right)^{\frac{1}{8}} \quad \forall t_{b} \geq t_{a} \geq 0 .
\end{aligned}
$$

An immediate consequence of $(2.61)$ is that

$$
\left\|\boldsymbol{U}_{(\sigma)}-\boldsymbol{U}_{(\sigma)}^{ \pm}\right\|_{\boldsymbol{L}^{\infty}\left(\Omega_{T}\right)} \leq C \tau^{\frac{1}{8}} .
$$


Now (2.58), (2.60) and (2.61) imply that the $\boldsymbol{C}_{x, t}^{\frac{1}{2}, \frac{1}{8}}\left(\bar{\Omega}_{T}\right)$ norm of $\boldsymbol{U}_{(\sigma)}$ is bounded independently of $h, \tau, \sigma$ and $T$. Hence, under the stated assumptions on $\tau(, \sigma)$, every sequence $\left\{\boldsymbol{U}_{(\sigma)}\right\}_{h}$ is uniformly bounded and equicontinuous on $\bar{\Omega}_{T}$, for any $T>0$. Therefore by the Arzelà-Ascoli theorem there exists a subsequence such that

$$
\boldsymbol{U}_{(\sigma)} \rightarrow \boldsymbol{u} \in \boldsymbol{C}_{x, t}^{\frac{1}{2}, \frac{1}{8}}\left(\bar{\Omega}_{T}\right) \quad \text { uniformly on } \bar{\Omega}_{T} \text { as } h \rightarrow 0
$$

and $\boldsymbol{u}(\cdot, t) \in \boldsymbol{K}_{\boldsymbol{m}}$. Moreover (2.58) implies that this same subsequence is such that

$$
\boldsymbol{U}_{(\sigma)} \rightarrow \boldsymbol{u} \quad \text { weakly in } L^{2}\left(0, T ; \boldsymbol{H}^{1}(\Omega)\right) \text { as } h \rightarrow 0 .
$$

For any $\boldsymbol{\eta} \in H^{1}\left(0, T ; \boldsymbol{H}^{1}(\Omega)\right)$ we choose $\boldsymbol{\chi} \equiv \boldsymbol{\pi}^{h} \boldsymbol{\eta}$ in (2.53a) and now analyse the subsequent terms. Firstly, we have that

$$
\int_{0}^{T}\left(\frac{\partial}{\partial t} \boldsymbol{U}_{(\sigma)}, \boldsymbol{\pi}^{h} \boldsymbol{\eta}\right)^{h} \mathrm{~d} t=-\int_{0}^{T}\left(\boldsymbol{U}_{(\sigma)}, \frac{\partial}{\partial t}\left(\boldsymbol{\pi}^{h} \boldsymbol{\eta}\right)\right)^{h} \mathrm{~d} t+\left(\boldsymbol{U}_{(\sigma)}(\cdot, T), \boldsymbol{\pi}^{h} \boldsymbol{\eta}(\cdot, T)\right)^{h}-\left(\boldsymbol{U}_{(\sigma)}(\cdot, 0), \boldsymbol{\pi}^{h} \boldsymbol{\eta}(\cdot, 0)\right)^{h} .
$$

Next we conclude using the regularity of $\boldsymbol{\eta},(2.19)$ and (2.63) that

$$
\int_{0}^{T}\left(\boldsymbol{U}_{(\sigma)}, \frac{\partial}{\partial t}\left(\boldsymbol{\pi}^{h} \boldsymbol{\eta}\right)\right)^{h} \mathrm{~d} t \rightarrow \int_{0}^{T}\left(\boldsymbol{u}, \frac{\partial}{\partial t} \boldsymbol{\eta}\right) \mathrm{d} t \quad \text { as } h \rightarrow 0 \quad \text { for all } \boldsymbol{\eta} \text { as above. }
$$

The bound (2.58) on $\boldsymbol{W}_{(\sigma)}^{+}$does not lead to the control of $W_{(\sigma), n}^{+}$on compact subsets of $\left\{u_{n}>0\right\}, n=1 \rightarrow N$, but only to the control of $W_{(\sigma), n}^{+}-W_{(\sigma), p}^{+}$on compact subsets of $\left\{u_{n}>0\right\} \cap\left\{u_{p}>0\right\}, n, p=1 \rightarrow N$; see (1.14b) and (2.5b). To do this, firstly we note that (1.14a) implies for all $\boldsymbol{\eta} \in L^{2}\left(0, T ; \boldsymbol{H}^{1}(\Omega)\right)$ that

$$
\int_{\Omega_{T}} L^{(\sigma)}\left(\boldsymbol{U}_{(\sigma)}^{-}\right) \frac{\partial}{\partial x} \boldsymbol{W}_{(\sigma)}^{+} \frac{\partial}{\partial x} \boldsymbol{\eta} \mathrm{d} x \mathrm{~d} t \equiv-\sum_{n=1}^{N} \sum_{p=1}^{N} \int_{\Omega_{T}} L_{n p}^{(\sigma)}\left(\boldsymbol{U}_{(\sigma)}^{-}\right) \frac{\partial}{\partial x} Z_{(\sigma,) n p}^{+} \frac{\partial}{\partial x} \eta_{n} \mathrm{~d} x \mathrm{~d} t ;
$$

where for notational convenience we have set

$$
Z_{(\sigma,) n p}^{+}:=W_{(\sigma,) n}^{+}-W_{(\sigma,) p}^{+} \quad n, p=1 \rightarrow N .
$$

In view of (1.14b), (2.5b), (2.58), (1.6), (1.13), (2.4a,b) and $\boldsymbol{U}_{(\sigma)}^{-} \in \boldsymbol{K}_{\boldsymbol{m}}^{h}$ we deduce for $n, p=1 \rightarrow N, n \neq p$, that

$$
\begin{aligned}
& \int_{\Omega_{T}}\left|L_{n p}^{(\sigma)}\left(\boldsymbol{U}_{(\sigma)}^{-}\right) \frac{\partial}{\partial x} Z_{(\sigma,) n p}^{+} \frac{\partial}{\partial x}\left(I-\pi^{h}\right) \eta_{n}\right| \mathrm{d} x \mathrm{~d} t \\
& \quad \leq\left\|\left[-L_{n p}^{(\sigma)}\left(\boldsymbol{U}_{(\sigma)}^{-}\right)\right]^{\frac{1}{2}} \frac{\partial}{\partial x} Z_{(\sigma,) n p}^{+}\right\|_{L^{2}\left(\Omega_{T}\right)}\left\|\left[-L_{n p}^{(\sigma)}\left(\boldsymbol{U}_{(\sigma)}^{-}\right)\right]^{\frac{1}{2}} \frac{\partial}{\partial x}\left(I-\pi^{h}\right) \eta_{n}\right\|_{L^{2}\left(\Omega_{T}\right)} \\
& \quad \leq C\left\|\left(I-\pi^{h}\right) \eta_{n}\right\|_{L^{2}\left(0, T ; H^{1}(\Omega)\right)} \quad \forall \eta_{n} \in L^{2}\left(0, T ; H^{1}(\Omega)\right) .
\end{aligned}
$$

For any $\delta>0$, we then set for $n=1 \rightarrow n$

$$
\begin{aligned}
D_{\delta, n}^{+} & :=\left\{(x, t) \in \bar{\Omega}_{T}: u_{n}(x, t)>\delta\right\} \\
\text { and } D_{\delta, n}^{+}(t) & :=\left\{x \in \bar{\Omega}: u_{n}(x, t)>\delta\right\} .
\end{aligned}
$$


For a fixed $\delta>0$, it follows from (2.63) and (2.62) that there exists a $h_{0}(\delta) \in \mathbb{R}^{+}$such that for $n=1 \rightarrow N$ and for all $h \leq h_{0}(\delta)$

$$
\begin{aligned}
0 & \leq U_{(\sigma,) n}^{ \pm}(x, t) \leq 2 \delta \quad \forall(x, t) \notin D_{\delta, n}^{+} \\
\text {and } \frac{1}{8} \delta_{1} & \leq U_{(\sigma,) n}^{ \pm}(x, t) \quad \forall(x, t) \in D_{\frac{\delta_{1}}{4}, n}^{+} \quad \delta_{1}=\delta \text { or } 2 \delta .
\end{aligned}
$$

By choosing $h_{0}(\delta)$ small enough, we can assume also, without loss of generality from assumption iii), in the case of $\left(\mathrm{P}_{\sigma}^{h, \tau}\right)$ that $\sigma(h) \leq \delta$ for all $h \leq h_{0}(\delta)$. On noting (1.14b), (2.5b), (2.58), (1.6), (1.13), (2.4a,b), and (2.71) we have for $n, p=1 \rightarrow N, n \neq p$, and for all $h \leq h_{0}(\delta)$ that

$$
\begin{aligned}
\left|\int_{\Omega_{T} \backslash\left(D_{\delta, n}^{+} \cap D_{\delta, p}^{+}\right)} L_{n p}^{(\sigma)}\left(\boldsymbol{U}_{(\sigma)}^{-}\right) \frac{\partial}{\partial x} Z_{(\sigma,) n p}^{+} \frac{\partial}{\partial x} \eta_{n} \mathrm{~d} x \mathrm{~d} t\right| \leq & \left\|\left[-L_{n p}^{(\sigma)}\left(\boldsymbol{U}_{(\sigma)}^{-}\right)\right]^{\frac{1}{2}}\right\|_{L^{\infty}\left(\Omega_{T} \backslash\left(D_{\delta, n}^{+} \cap D_{\delta, p}^{+}\right)\right)} \\
& \times\left\|\left[-L_{n p}^{(\sigma)}\left(\boldsymbol{U}_{(\sigma)}^{-}\right)\right]^{\frac{1}{2}} \frac{\partial}{\partial x} Z_{(\sigma,) n}^{+}\right\|\left\|_{L^{2}\left(\Omega_{T}\right)}\right\| \eta_{n} \|_{L^{2}\left(0, T ; H^{1}(\Omega)\right)} \\
\leq & C \delta^{\frac{1}{2}}\left\|\eta_{n}\right\|_{L^{2}\left(0, T ; H^{1}(\Omega)\right)} \quad \forall \eta_{n} \in L^{2}\left(0, T ; H^{1}(\Omega)\right)
\end{aligned}
$$

and

$$
C \delta^{2} \int_{D_{\frac{\delta}{2}, n}^{+} \cap D_{\frac{\delta}{2}, p}^{+}}\left(\frac{\partial}{\partial x} Z_{(\sigma,) n p}^{+}\right)^{2} \mathrm{~d} x \mathrm{~d} t \leq \int_{D_{\frac{\delta}{2}, n}^{+} \cap D_{\frac{\delta}{2}, p}^{+}}\left[-L_{n p}^{(\sigma)}\left(\boldsymbol{U}_{(\sigma)}^{-}\right)\right]\left(\frac{\partial}{\partial x} Z_{(\sigma,) n p}^{+}\right)^{2} \mathrm{~d} x \mathrm{~d} t \leq C .
$$

In what follows we want to relate $Z_{(\sigma,) n p}^{+}$to $U_{(\sigma,) n}^{ \pm}$and $U_{(\sigma,) p}^{ \pm}$on the sets $D_{\delta, n}^{+} \cap D_{\delta, p}^{+}$. From (2.71) we have for all $h \leq h_{0}(\delta)$ and for a.e. $t \in(0, T)$ that

$$
\begin{aligned}
\chi(\cdot, t) & \equiv \boldsymbol{U}_{(\sigma)}^{+}(\cdot, t) \pm \frac{1}{8} \delta \frac{\eta^{h}(\cdot, t)}{\left\|\eta^{h}(\cdot, t)\right\|_{0, \infty}} e^{n} \in \boldsymbol{K}^{h} \\
& \forall \eta^{h} \in L^{2}\left(0, T ; S^{h}\right) \quad \text { with } \operatorname{supp}\left(\eta^{h}\right) \subset D_{\frac{\delta}{4}, n}^{+}, \quad n=1 \rightarrow N .
\end{aligned}
$$

Choosing such $\chi$ in (2.53b) yields for $n=1 \rightarrow N$ and for all $h \leq h_{0}(\delta)$ that

$$
\begin{aligned}
& \int_{0}^{T}\left[\gamma\left(\frac{\partial}{\partial x} U_{(\sigma,) n}^{+}, \frac{\partial}{\partial x} \eta^{h}\right)+\left(\psi_{1}^{\prime}\left(U_{(\sigma,) n}^{+}\right)-\right.\right.\left.\left.\left\{A^{-} \boldsymbol{U}_{(\sigma)}^{+}+A^{+} \boldsymbol{U}_{(\sigma)}^{-}\right\}_{n}, \eta^{h}\right)^{h}-\left(W_{(\sigma,) n}^{+}, \eta^{h}\right)^{h}\right] \mathrm{d} t=0 \\
& \forall \eta^{h} \in L^{2}\left(0, T ; S^{h}\right) \quad \text { with } \operatorname{supp}\left(\eta^{h}\right) \subset D_{\frac{\delta}{4}, n}^{+} .
\end{aligned}
$$

Hence from (2.75), it follows for $n, p=1 \rightarrow N$ and for all $h \leq h_{0}(\delta)$ that

$$
\begin{aligned}
& \int_{0}^{T}\left[\gamma\left(\frac{\partial}{\partial x}\left(U_{(\sigma,) n}^{+}-U_{(\sigma,) p}^{+}\right), \frac{\partial}{\partial x} \eta^{h}\right)+\left(\left[\psi_{1}^{\prime}\left(U_{(\sigma,) n}^{+}\right)-\psi_{1}^{\prime}\left(U_{(\sigma,) p}^{+}\right)\right], \eta^{h}\right)^{h}\right. \\
& \left.-\left(\left[\left\{A^{-} \boldsymbol{U}_{(\sigma)}^{+}+A^{+} \boldsymbol{U}_{(\sigma)}^{-}\right\}_{n}-\left\{A^{-} \boldsymbol{U}_{(\sigma)}^{+}+A^{+} \boldsymbol{U}_{(\sigma)}^{-}\right\}_{p}\right]+Z_{(\sigma,) n p}^{+}, \eta^{h}\right)^{h}\right] \mathrm{d} t=0 \\
& \forall \eta^{h} \in L^{2}\left(0, T ; S^{h}\right) \quad \operatorname{with} \operatorname{supp}\left(\eta^{h}\right) \subset D_{\frac{\delta}{4}, n}^{+} \cap D_{\frac{\delta}{4}, p}^{+} .
\end{aligned}
$$

For any $t \in[0, T]$ and for $n=1 \rightarrow N$, we choose a cut-off function $\alpha_{\delta, n}(\cdot, t) \in C^{\infty}(\bar{\Omega})$ such that

$$
\begin{array}{ll}
\alpha_{\delta, n}(\cdot, t) \equiv 1 \quad \text { on } D_{\delta, n}^{+}(t), \quad 0 \leq \alpha_{\delta, n}(\cdot, t) \leq 1 \quad \text { on } D_{\frac{\delta}{2}, n}^{+}(t) \backslash D_{\delta, n}^{+}(t), \\
\alpha_{\delta, n}(\cdot, t) \equiv 0 & \text { on } \bar{\Omega} \backslash D_{\frac{\delta}{2}, n}^{+}(t) \quad \text { and } \quad\left|\frac{\partial}{\partial x} \alpha_{\delta, n}(\cdot, t)\right| \leq C \delta^{-2} .
\end{array}
$$


This last property can be achieved since for $y_{1}, y_{2} \in \bar{\Omega}$ such that $u_{n}\left(y_{1}, t\right)=\frac{1}{2} \delta$ and $u_{n}\left(y_{2}, t\right)=\delta$ we have from (2.63) that $\frac{1}{2} \delta=\left|u_{n}\left(y_{2}, t\right)-u_{n}\left(y_{1}, t\right)\right| \leq C\left|y_{2}-y_{1}\right|^{\frac{1}{2}}$. It follows from (2.21) and (2.77) that there exists a $h_{1}(\delta) \leq h_{0}(\delta)$ such that for $n, p=1 \rightarrow N, n \neq p$,

$$
\operatorname{supp}\left(Q_{2}^{h}\left[\alpha_{\delta, n p}^{2} Z_{(\sigma,) n p}^{+}\right]\right) \subset D_{\frac{\delta}{4}, n}^{+} \cap D_{\frac{\delta}{4}, p}^{+} \quad \forall h \leq h_{1}(\delta) ;
$$

where $\alpha_{\delta, n p}:=\alpha_{\delta, n} \alpha_{\delta, p}$. It then follows from (2.2), (2.78), (2.75), (2.20), $\psi_{1} \in C^{1}([0,1]), \boldsymbol{U}_{(\sigma)} \in \boldsymbol{K}_{\boldsymbol{m}}^{h}$ and (2.77) that for $n \neq p$ and for all $h \leq h_{1}(\delta)$

$$
\begin{aligned}
\int_{\Omega_{T}} \alpha_{\delta, n p}^{2}\left(Z_{(\sigma,) n p}^{+}\right)^{2} \mathrm{~d} x \mathrm{~d} t= & \int_{0}^{T}\left(Z_{(\sigma,) n p}^{+}, Q_{2}^{h}\left[\alpha_{\delta, n p}^{2} Z_{(\sigma,) n p}^{+}\right]\right)^{h} \mathrm{~d} t \\
= & \int_{0}^{T}\left[\gamma\left(\frac{\partial}{\partial x}\left(U_{(\sigma,) n}^{+}-U_{(\sigma,) p}^{+}\right), \frac{\partial}{\partial x} Q_{2}^{h}\left[\alpha_{\delta, n p}^{2} Z_{(\sigma,) n p}^{+}\right]\right)\right. \\
& -\left(\left[\left\{A^{-} \boldsymbol{U}_{(\sigma)}^{+}+A^{+} \boldsymbol{U}_{(\sigma)}^{-}\right\}_{n}-\left\{A^{-} \boldsymbol{U}_{(\sigma)}^{+}+A^{+} \boldsymbol{U}_{(\sigma)}^{-}\right\}_{p}\right], Q_{2}^{h}\left[\alpha_{\delta, n p}^{2} Z_{(\sigma,) n p}^{+}\right]\right)^{h} \\
& \left.+\left(\left[\psi_{1}^{\prime}\left(U_{(\sigma,) n}^{+}\right)-\psi_{1}^{\prime}\left(U_{(\sigma,) p}^{+}\right)\right], Q_{2}^{h}\left[\alpha_{\delta, n p}^{2} Z_{(\sigma,) n p}^{+}\right]\right)^{h}\right] \mathrm{d} t \\
\leq & C\left\|\boldsymbol{U}_{(\sigma)}^{+}\right\|_{L^{2}\left(0, T ; \boldsymbol{H}^{1}(\Omega)\right)}\left\|\frac{\partial}{\partial x}\left(\alpha_{\delta, n p}^{2} Z_{(\sigma,) n p}^{+}\right)\right\|_{L^{2}\left(\Omega_{T}\right)}+C\left\|\alpha_{\delta, n p} Z_{(\sigma,) n p}^{+}\right\|_{L^{2}\left(\Omega_{T}\right)} \\
\leq & C\left(\delta^{-1}\right)\left(\left\|\boldsymbol{U}_{(\sigma)}^{+}\right\|_{L^{2}\left(0, T ; \boldsymbol{H}^{1}(\Omega)\right)}+1\right) \\
& \times\left[\left\|\frac{\partial}{\partial x} Z_{(\sigma,) n p}^{+}\right\|_{L^{2}\left(D_{\frac{\delta}{2}, n}^{+} \cap D_{\frac{\delta}{2}, p}^{+}\right)}+\left\|\alpha_{\delta, n p} Z_{(\sigma,) n p}^{+}\right\|_{L^{2}\left(\Omega_{T}\right)}\right] .
\end{aligned}
$$

Applying Young's inequality then gives

$$
\int_{\Omega_{T}} \alpha_{\delta, n p}^{2}\left(Z_{(\sigma,) n p}^{+}\right)^{2} \mathrm{~d} x \mathrm{~d} t \leq C\left(\delta^{-1}\right)\left[1+\left\|\boldsymbol{U}_{(\sigma)}^{+}\right\|_{L^{2}\left(0, T ; \boldsymbol{H}^{1}(\Omega)\right)}^{2}+\left\|\frac{\partial}{\partial x} Z_{(\sigma,) n p}^{+}\right\|_{L^{2}\left(D_{\frac{\delta}{2}, n}^{+} \cap D_{\frac{\delta}{2}, p}^{+}\right)}^{2}\right] .
$$

Therefore combining (2.73), (2.80) and (2.58) we have for $n, p=1 \rightarrow N, n \neq p$, that

$$
\left\|Z_{(\sigma,) n p}^{+}\right\|_{L^{2}\left(0, T ; H^{1}\left(D_{\delta, n}^{+}(t) \cap D_{\delta, p}^{+}(t)\right)\right)} \leq C\left(\delta^{-1}\right) \quad \forall h \leq h_{1}(\delta) .
$$

This implies the existence of a subsequence and $z_{n p} \in L^{2}\left(0, T ; H^{1}\left(D_{\delta, n}^{+}(t) \cap D_{\delta, p}^{+}(t)\right)\right)$ such that

$$
\begin{gathered}
Z_{(\sigma,) n p}^{+} \rightarrow z_{n p}, \quad \frac{\partial}{\partial x} Z_{(\sigma,) n p}^{+} \rightarrow \frac{\partial}{\partial x} z_{n p} \quad \text { weakly in } L^{2}\left(D_{\delta, n}^{+} \cap D_{\delta, p}^{+}\right) \text {as } h \rightarrow 0 \\
n, p=1 \rightarrow N, n \neq p .
\end{gathered}
$$

Next it follows from (2.73), (1.6), (1.13), (2.4a,b), $\boldsymbol{u}(\cdot, t) \in \boldsymbol{K}_{\boldsymbol{m}}$ and $\boldsymbol{U}_{(\sigma)}(\cdot, t) \in \boldsymbol{K}_{\boldsymbol{m}}^{h}$ that for $n, p=1 \rightarrow N$, $n \neq p$,

$$
\begin{aligned}
& \left|\int_{D_{\delta, n}^{+} \cap D_{\delta, p}^{+}}\left[L_{n p}^{(\sigma)}\left(\boldsymbol{U}_{(\sigma)}^{-}\right)-L_{n p}(\boldsymbol{u})\right] \frac{\partial}{\partial x} Z_{(\sigma,) n p}^{+} \frac{\partial}{\partial x} \eta_{n} \mathrm{~d} x \mathrm{~d} t\right| \\
& \quad \leq\left\|L_{n p}(\boldsymbol{u})-L_{n p}^{(\sigma)}\left(\boldsymbol{U}_{(\sigma)}^{-}\right)\right\|_{L^{\infty}\left(\Omega_{T}\right)}\left\|\frac{\partial}{\partial x} Z_{(\sigma,) n p}^{+}\right\|_{L^{2}\left(D_{\delta, n}^{+} \cap D_{\delta, p}^{+}\right)}\left\|\eta_{n}\right\|_{L^{2}\left(0, T ; H^{1}(\Omega)\right)} \\
& \quad \leq C\left(\delta^{-1}\right)\left\|\eta_{n}\right\|_{L^{2}\left(0, T ; H^{1}(\Omega)\right)} \sum_{m=1}^{N}\left\|l_{m}\left(u_{m}\right)-l_{m}^{(\sigma)}\left(U_{(\sigma,) m}^{-}\right)\right\|_{L^{\infty}\left(\Omega_{T}\right)} ;
\end{aligned}
$$


which will converge to 0 as $h \rightarrow 0$ on noting (2.63), (2.62), assumption iii) and that $l_{m} \in C([0,1]), m=1 \rightarrow N$.

Combining (2.69), (2.83) and (2.82) and noting (2.18), (2.63) and (2.62) yields for $n, p=1 \rightarrow N, n \neq p$, and for all $\eta_{n} \in L^{2}\left(0, T ; H^{1}(\Omega)\right)$ that

$$
\int_{D_{\delta, n}^{+} \cap D_{\delta, p}^{+}} L_{n p}^{(\sigma)}\left(\boldsymbol{U}_{(\sigma)}^{-}\right) \frac{\partial}{\partial x} Z_{(\sigma,) n p}^{+} \frac{\partial}{\partial x}\left(\pi^{h} \eta_{n}\right) \mathrm{d} x \mathrm{~d} t \rightarrow \int_{D_{\delta, n}^{+} \cap D_{\delta, p}^{+}} L_{n p}(\boldsymbol{u}) \frac{\partial}{\partial x} z_{n p} \frac{\partial}{\partial x} \eta_{n} \mathrm{~d} x \mathrm{~d} t \quad \text { as } h \rightarrow 0 .
$$

Moreover, by (2.18), (2.64), (2.59) and assumption iii) we have for all $\boldsymbol{\eta} \in L^{2}\left(0, T ; \boldsymbol{H}^{1}(\Omega)\right)$ that

$$
\int_{0}^{T}\left(\frac{\partial}{\partial x} \boldsymbol{U}_{(\sigma)}^{+}, \frac{\partial}{\partial x}\left(\boldsymbol{\pi}^{h} \boldsymbol{\eta}\right)\right) \mathrm{d} t \rightarrow \int_{0}^{T}\left(\frac{\partial}{\partial x} \boldsymbol{u}, \frac{\partial}{\partial x} \boldsymbol{\eta}\right) \mathrm{d} t \quad \text { as } h \rightarrow 0 .
$$

Using (2.1), (2.17) and (2.81) we deduce for $n, p=1 \rightarrow N, n \neq p$, that

$$
\begin{gathered}
\left|\int_{0}^{T}\left[\left(Z_{(\sigma,) n p}^{+}, \pi^{h} \eta_{n}\right)^{h}-\left(Z_{(\sigma,) n p}^{+}, \eta_{n}\right)\right] \mathrm{d} t\right| \equiv\left|\int_{\Omega_{T}}\left(I-\pi^{h}\right)\left(Z_{(\sigma,) n p}^{+} \eta_{n}\right) \mathrm{d} x \mathrm{~d} t\right| \\
\leq C h \int_{\Omega_{T}}\left|\frac{\partial}{\partial x}\left(Z_{(\sigma,) n p}^{+} \eta_{n}\right)\right| \mathrm{d} x \mathrm{~d} t \\
\leq C h\left\|Z_{(\sigma,) n p}^{+}\right\|_{L^{2}\left(0, T ; H^{1}\left(D_{\delta, n}^{+}(t) \cap D_{\delta, p}^{+}(t)\right)\right)}\left\|\eta_{n}\right\|_{L^{2}\left(0, T ; H^{1}(\Omega)\right)} \\
\leq C\left(\delta^{-1}\right) h\left\|\eta_{n}\right\|_{L^{2}\left(0, T ; H^{1}(\Omega)\right)} \\
\quad \forall \eta_{n} \in L^{2}\left(0, T ; H^{1}(\Omega)\right) \text { with } \operatorname{supp}\left(\eta_{n}\right) \subset D_{\delta, n}^{+} \cap D_{\delta, p}^{+} .
\end{gathered}
$$

Noting that $\psi_{1} \in C^{1}([0,1])$ and using $(2.1),(2.17),(2.54),(2.19)$ and $(2.13)$ yields that

$$
\begin{aligned}
& \left|\int_{0}^{T}\left[\left(\psi_{1}^{\prime}\left(U_{(\sigma,) n}^{+}\right), \pi^{h} \eta_{n}\right)^{h}-\left(\psi_{1}^{\prime}\left(u_{n}\right), \eta_{n}\right)\right] \mathrm{d} t\right| \leq \int_{0}^{T}\left|\left(\psi_{1}^{\prime}\left(U_{(\sigma,) n}^{+}\right)-\psi_{1}^{\prime}\left(u_{n}\right), \pi^{h} \eta_{n}\right)^{h}\right| \mathrm{d} t \\
& \quad+\int_{0}^{T}\left|\left(\psi_{1}^{\prime}\left(u_{n}\right), \pi^{h} \eta_{n}\right)^{h}-\left(\psi_{1}^{\prime}\left(u_{n}\right), \eta_{n}\right)\right| \mathrm{d} t \rightarrow 0 \quad \text { as } h \rightarrow 0, \quad \forall \eta_{n} \in L^{2}\left(0, T ; H^{1}(\Omega)\right) .
\end{aligned}
$$

Using a similar argument for the remaining terms and combining (2.86), (2.82) and (2.87) implies for $n, p=$ $1 \rightarrow N, n \neq p$, that

$$
\begin{aligned}
\int_{0}^{T}[ & \left(\left[\psi_{1}^{\prime}\left(U_{(\sigma,) n}^{+}\right)-\psi_{1}^{\prime}\left(U_{(\sigma,) p}^{+}\right)\right]-Z_{(\sigma,) n p}^{+}, \pi^{h} \eta_{n}\right)^{h} \\
& \left.-\left(\left\{A^{-} \boldsymbol{U}_{(\sigma)}^{+}+A^{+} \boldsymbol{U}_{(\sigma)}^{-}\right\}_{n}-\left\{A^{-} \boldsymbol{U}_{(\sigma)}^{+}+A^{+} \boldsymbol{U}_{(\sigma)}^{-}\right\}_{p}, \pi^{h} \eta_{n}\right)^{h}\right] \mathrm{d} t \\
& \rightarrow \int_{0}^{T}\left[\left(\left[\psi_{1}^{\prime}\left(u_{n}\right)-\psi_{1}^{\prime}\left(u_{p}\right)\right]-z_{n p}-\left[\{A \boldsymbol{u}\}_{n}-\{A \boldsymbol{u}\}_{p}\right], \eta_{n}\right)^{h}\right] \mathrm{d} t \\
& \quad \text { as } h \rightarrow 0, \quad \forall \eta_{n} \in L^{2}\left(0, T ; H^{1}(\Omega)\right) \quad \text { with } \operatorname{supp}\left(\eta_{n}\right) \subset D_{\delta, n}^{+} \cap D_{\delta, p}^{+} .
\end{aligned}
$$

Combining (2.85) and (2.88) and noting (2.76) yields for $n, p=1 \rightarrow N, n \neq p$, that

$$
\begin{array}{r}
\int_{D_{\delta, n}^{+} \cap D_{\delta, p}^{+}}\left[\left(\left[\psi_{1}^{\prime}\left(u_{n}\right)-\psi_{1}^{\prime}\left(u_{p}\right)\right]-\left[\{A \boldsymbol{u}\}_{n}-\{A \boldsymbol{u}\}_{p}\right]-z_{n p}\right) \eta_{n}+\gamma \frac{\partial}{\partial x}\left(u_{n}-u_{p}\right) \frac{\partial}{\partial x} \eta_{n}\right] \mathrm{d} x \mathrm{~d} t=0 \\
\forall \eta_{n} \in L^{2}\left(0, T ; H^{1}(\Omega)\right) \text { with } \operatorname{supp}\left(\eta_{n}\right) \subset D_{\delta, n}^{+} \cap D_{\delta, p}^{+} .
\end{array}
$$


This uniquely defines $z_{n p}$ in terms of $\boldsymbol{u}$ on the set $D_{\delta, n}^{+} \cap D_{\delta, p}^{+}$. Repeating (2.84) for all $\delta>0$ and noting (2.72) and (2.18) yields for $n, p=1 \rightarrow N, n \neq p$, and for all $\eta_{n} \in L^{2}\left(0, T ; H^{1}(\Omega)\right)$ that

$$
\int_{\Omega_{T}} L_{n p}^{(\sigma)}\left(\boldsymbol{U}_{(\sigma)}^{-}\right) \frac{\partial}{\partial x} Z_{(\sigma,) n p}^{+} \frac{\partial}{\partial x}\left(\pi^{h} \eta_{n}\right) \mathrm{d} x \mathrm{~d} t \rightarrow \int_{D_{0, n}^{+} \cap D_{0, p}^{+}} L_{n p}(\boldsymbol{u}) \frac{\partial}{\partial x} z_{n p} \frac{\partial}{\partial x} \eta_{n} \mathrm{~d} x \mathrm{~d} t \quad \text { as } h \rightarrow 0 .
$$

Combining (2.53a), (2.65), (2.66), (2.67), (2.90) and arguing similarly as in (2.87) by using (2.54), (2.19), (2.13) and assumption i) we conclude for all $\boldsymbol{\eta} \in H^{1}\left(0, T ; \boldsymbol{H}^{1}(\Omega)\right)$ that

$$
(\boldsymbol{u}(\cdot, T), \boldsymbol{\eta}(\cdot, T))-\left(\boldsymbol{u}^{0}(\cdot), \boldsymbol{\eta}(\cdot, 0)\right)-\int_{0}^{T}\left(\boldsymbol{u}, \frac{\partial}{\partial t} \boldsymbol{\eta}\right) \mathrm{d} t-\sum_{n=1}^{N} \sum_{p=1}^{N} \int_{D_{0, n}^{+} \cap D_{0, p}^{+}} L_{n p}(\boldsymbol{u}) \frac{\partial}{\partial x} z_{n p} \frac{\partial}{\partial x} \eta_{n} \mathrm{~d} x \mathrm{~d} t=0 .
$$

In the above we have also noted that (2.56) implies that $z_{n n} \equiv 0$ on $\Omega_{T}, n=1 \rightarrow N$.

As $\left\{\left[-L_{n p}^{(\sigma)}\left(\boldsymbol{U}_{(\sigma)}^{-}\right)\right]^{\frac{1}{2}} \frac{\partial}{\partial x} Z_{(\sigma,) n p}^{+}\right\}_{h>0}$ is uniformly bounded in $L^{2}\left(\Omega_{T}\right), n \neq p$, see (2.72); it follows from the above that $\left[-L_{n p}(\boldsymbol{u})\right]^{\frac{1}{2}} \frac{\partial}{\partial x} z_{n p} \in \boldsymbol{L}^{2}\left(D_{0, n}^{+} \cap D_{0, p}^{+}\right), n \neq p$. Hence we conclude from (2.91) that $\boldsymbol{u} \in$ $H^{1}\left(0, T ;\left(\boldsymbol{H}^{1}(\Omega)\right)^{\prime}\right)$. Therefore combining the above results, repeating (2.89) for all $\delta>0$ yields that

$\boldsymbol{u} \in L^{\infty}\left(0, T ; \boldsymbol{H}^{1}(\Omega)\right) \cap H^{1}\left(0, T ;\left(\boldsymbol{H}^{1}(\Omega)\right)^{\prime}\right) \cap \boldsymbol{C}_{x, t}^{\frac{1}{2}, \frac{1}{8}}\left(\bar{\Omega}_{T}\right)$ and $z_{n p} \in L_{l o c}^{2}\left(D_{0, n}^{+} \cap D_{0, p}^{+}\right)$, with $\frac{\partial}{\partial x} z_{n p} \in L_{l o c}^{2}\left(D_{0, n}^{+} \cap\right.$ $\left.D_{0, p}^{+}\right), n, p=1 \rightarrow N$, are such that $\boldsymbol{u}(\cdot, 0)=\boldsymbol{u}^{0}(\cdot)$ and

$$
\begin{gathered}
\int_{0}^{T}\left\langle\frac{\partial}{\partial t} u_{n}, \eta_{n}\right\rangle \mathrm{d} t-\sum_{p=1}^{N} \int_{D_{0, n}^{+} \cap D_{0, p}^{+}} L_{n p}(\boldsymbol{u}) \frac{\partial}{\partial x} z_{n p} \frac{\partial}{\partial x} \eta_{n} \mathrm{~d} x \mathrm{~d} t=0 \\
\forall \eta_{n} \in L^{2}\left(0, T ; H^{1}(\Omega)\right), \quad n=1 \rightarrow N ; \\
\int_{D_{0, n}^{+} \cap D_{0, p}^{+}}\left[\left(\left[\psi_{1}^{\prime}\left(u_{n}\right)-\psi_{1}^{\prime}\left(u_{p}\right)\right]-\left[\{A \boldsymbol{u}\}_{n}-\{A \boldsymbol{u}\}_{p}\right]-z_{n p}\right) \eta_{n}+\gamma \frac{\partial}{\partial x}\left(u_{n}-u_{p}\right) \frac{\partial}{\partial x} \eta_{n}\right] \mathrm{d} x \mathrm{~d} t=0 \\
\forall \eta_{n} \in L^{2}\left(0, T ; H^{1}(\Omega)\right) \text { with } \operatorname{supp}\left(\eta_{n}\right) \subset D_{0, n}^{+} \cap D_{0, p}^{+}, \quad n, p=1 \rightarrow N .
\end{gathered}
$$

Hence we have established the desired results $(2.57 \mathrm{a}, \mathrm{b})$.

By modifying the mobility matrix $L^{\sigma}$ in our approximation $\left(\mathrm{P}_{\sigma}^{h, \tau}\right)$ it is possible to ensure that the limit function $\boldsymbol{u}$ in Theorem 2.2 above is such that $\boldsymbol{u} \in L^{2}\left(0, T ; \boldsymbol{H}^{2}(\Omega)\right)$. This idea has been introduced in Zhornitskaya and Bertozzi [30] and Grün and Rumpf [24] for the thin film equation

$$
\frac{\partial}{\partial t} u+\frac{\partial}{\partial x}\left(b(u) \frac{\partial^{3}}{\partial x^{3}} u\right)=0
$$

where $b(u):=|u|^{p}$ for a given $p \in(0, \infty)$. We adapt their approach here to obtain a discrete version of the required entropy bound in Elliott and Garcke [19, Lem. 10]. For any $\sigma \in(0,1)$, we introduce $f_{n}^{\sigma}, F_{n}^{\sigma}:[0,1] \rightarrow \mathbb{R}$, $n=1 \rightarrow N$, such that

$$
f_{n}^{\sigma}(s):=-\int_{s}^{1}\left[l_{n}^{\sigma}(r)\right]^{-1} \mathrm{~d} r, \quad F_{n}^{\sigma}(s):=-\int_{s}^{1} f_{n}^{\sigma}(r) \mathrm{d} r \quad \forall s \in[0,1],
$$


where $l_{n}^{\sigma}$ is defined by (2.4b). It follows immediately from the above definitions that for $n=1 \rightarrow N$

$$
\begin{aligned}
\left(F_{n}^{\sigma}\right)^{\prime}(s)=f_{n}^{\sigma}(s), & \left(F_{n}^{\sigma}\right)^{\prime \prime}(s)=\left(f_{n}^{\sigma}\right)^{\prime}(s)=\left[l_{n}^{\sigma}(s)\right]^{-1} \quad \forall s \in[0,1], \\
l_{\min }^{-1}(s \ln s+1-s) \geq & F_{n}^{\sigma_{1}}(s) \\
\geq & F_{n}^{\sigma_{2}}(s) \geq 0 \quad \forall s \in[0,1], \quad \forall \sigma_{2}>\sigma_{1}>0, \\
\sigma^{-1}\left(s_{2}-s_{1}\right)^{2} & \geq\left(\left(F_{n}^{\sigma}\right)^{\prime}\left(s_{2}\right)-\left(F_{n}^{\sigma}\right)^{\prime}\left(s_{1}\right)\right)\left(s_{2}-s_{1}\right) \\
\geq & \left(l_{\max }+\sigma\right)^{-1}\left(s_{2}-s_{1}\right)^{2} \geq 0 \quad \forall s_{2}, s_{1} \in[0,1] .
\end{aligned}
$$

Hence $F_{n}^{\sigma}$ is a nonnegative convex function.

Similarly to Zhornitskaya and Bertozzi [30] and Grün and Rumpf [24], we introduce $\widehat{l}_{n}^{\sigma}: \widehat{S}^{h}:=\left\{\chi \in S^{h}\right.$ : $\left.\chi\left(\boldsymbol{x}_{j}\right) \in[0,1], \forall j \in J\right\} \rightarrow L^{\infty}(\Omega), n=1 \rightarrow N$, which is piecewise constant on $\mathcal{T}^{h}$. In the case $d=1$ given $q^{h} \in \widehat{S}^{h}$ and $\kappa \in \mathcal{T}^{h}$ having vertices $x_{i}$ and $x_{j}$, we set

$$
\left.\widehat{l}_{n}^{\sigma}\left(q^{h}\right)\right|_{\kappa}:=\left\{\begin{array}{cc}
\frac{q^{h}\left(x_{i}\right)-q^{h}\left(x_{j}\right)}{\left(F_{n}^{\sigma}\right)^{\prime}\left(q^{h}\left(x_{i}\right)\right)-\left(F_{n}^{\sigma}\right)^{\prime}\left(q^{h}\left(x_{j}\right)\right)} \equiv l_{n}^{\sigma}\left(q^{h}\left((1-\mu) x_{i}+\mu x_{j}\right)\right) \\
\text { for some } \mu \in(0,1) & \text { if } q^{h}\left(x_{i}\right) \neq q^{h}\left(x_{j}\right), \\
l_{n}^{\sigma}\left(q^{h}\left(x_{j}\right)\right) & \text { if } q^{h}\left(x_{i}\right)=q^{h}\left(x_{j}\right) .
\end{array} .\right.
$$

Clearly the piecewise constant construction in (2.95) has the property that for any $\sigma \in(0,1)$ and for $n=1 \rightarrow N$

$$
\widehat{l}_{n}^{\sigma}\left(q^{h}\right) \frac{d}{d x} \pi^{h}\left[\left(F_{n}^{\sigma}\right)^{\prime}\left(q^{h}\right)\right]=\frac{d}{d x} q^{h} \quad \text { a.e. in } \Omega, \quad \forall q^{h} \in \widehat{S}^{h} .
$$

We then introduce the $N \times N$ matrix $\widehat{L}^{\sigma}$, where for all $\sigma \in(0,1)$ and $\boldsymbol{q}^{h} \in \boldsymbol{K}_{\boldsymbol{m}}^{h}$

$$
\begin{aligned}
\left\{\widehat{L}^{\sigma}\left(\boldsymbol{q}^{h}\right)\right\}_{n p} \equiv \widehat{L}_{n p}^{\sigma}\left(\boldsymbol{q}^{h}\right) & :=\widehat{l}_{n}^{\sigma}\left(q_{n}^{h}\right)\left(\delta_{n p}-\left(\mathbf{1}^{T} \widehat{\boldsymbol{l}}^{\sigma}\left(\boldsymbol{q}^{h}\right)\right)^{-1} \widehat{l}_{p}^{\sigma}\left(q_{p}^{h}\right)\right) \\
\text { and } \quad\left\{\widehat{\boldsymbol{l}}^{\sigma}\left(\boldsymbol{q}^{h}\right)\right\}_{n} & :=\widehat{l}_{n}^{\sigma}\left(q_{n}^{h}\right), \quad n=1 \rightarrow N
\end{aligned}
$$

Similarly to (2.7), it follows that

$$
L_{\min }^{\sigma}\|\boldsymbol{\eta}\|^{2} \leq \boldsymbol{\eta}^{T} \widehat{L}^{\sigma}\left(\boldsymbol{q}^{h}(\boldsymbol{x})\right) \boldsymbol{\eta} \quad \forall \boldsymbol{q}^{h} \in \boldsymbol{K}_{\boldsymbol{m}}^{h}, \quad \boldsymbol{x} \in \bar{\Omega}, \quad \boldsymbol{\eta} \in \mathcal{M}(0) .
$$

We introduce also $F^{\sigma}:[0,1]^{N} \rightarrow \mathbb{R}$ such that

$$
F^{\sigma}(s):=\sum_{n=1}^{N} F_{n}^{\sigma}\left(s_{n}\right) \quad \forall s \in[0,1]^{N} .
$$

In addition we introduce the "discrete Laplacian" operator $\boldsymbol{\Delta}^{h}: \boldsymbol{S}^{h} \rightarrow \boldsymbol{S}^{h}$ such that $\left\{\boldsymbol{\Delta}^{h} \boldsymbol{v}^{h}\right\}_{n}:=\Delta^{h} v_{n}^{h}$ for all $\boldsymbol{v}^{h} \in \boldsymbol{S}^{h}$, where $\Delta^{h}: S^{h} \rightarrow S^{h}$ satisfies

$$
\left(\Delta^{h} v^{h}, \chi\right)^{h}=-\left(\nabla v^{h}, \nabla \chi\right) \quad \forall \chi \in S^{h} .
$$

Finally, we need a further restriction on the mesh. We modify our assumption (A) to

( $\widetilde{\mathbf{A}})$ In addition to the assumption (A), we assume for all $h>0$ that $\mathcal{T}^{h}$ is an acute partitioning; that is for (i) $d=2$ the angle of any triangle does not exceed $\frac{\pi}{2}$, (ii) $d=3$ the angle between any two faces of the same tetrahedron does not exceed $\frac{\pi}{2}$. 
This acuteness assumption yields that

$$
\int_{\kappa} \nabla \beta_{i} \cdot \nabla \beta_{j} \mathrm{~d} \boldsymbol{x} \leq 0 \quad i \neq j, \quad \forall \kappa \in \mathcal{T}^{h}
$$

Theorem 2.3. We consider the approximation $\left(P_{\sigma}^{h, \tau}\right)$ with the mobility matrix $L^{\sigma}$ replaced by $\widehat{L}^{\sigma}$. Then the results of Theorems 2.1 and 2.2 remain true under their stated assumptions with $L^{\sigma}$ replaced by $\widehat{L}^{\sigma}$.

Furthermore, under the assumptions of Theorem 2.1 with (A) replaced by $(\widetilde{A})$ it follows that

$$
\max _{k=1 \rightarrow K}\left(F^{\sigma}\left(\boldsymbol{U}_{\sigma}^{k}\right), 1\right)^{h}+\sum_{k=1}^{K} \gamma \tau_{k}\left|\boldsymbol{\Delta}^{h} \boldsymbol{U}_{\sigma}^{k}\right|_{h}^{2} \leq\left(F^{\sigma}\left(\boldsymbol{U}_{\sigma}^{0}\right), 1\right)^{h}+C \sigma^{-1} \tau^{\frac{1}{2}}\left[\left|\boldsymbol{U}_{\sigma}^{0}\right|_{1}^{2}+1\right]+C .
$$

Moreover, under the assumptions of Theorem 2.2 with the additional constraint that $\tau \leq C \sigma^{2}$ as $h \rightarrow 0$ we have that the limit function $\boldsymbol{u}$ lies in $L^{2}\left(0, T ; \boldsymbol{H}^{2}(\Omega)\right)$.

Proof. It is easily deduced from $(2.97 \mathrm{a}, \mathrm{b})$ and (2.95) that the results of Theorems 2.1 and 2.2 remain true under their stated assumptions with $L^{\sigma}$ replaced by $\widehat{L}^{\sigma}$.

Choosing $\chi \equiv \boldsymbol{\pi}^{h}\left[\boldsymbol{D} F^{\sigma}\left(\boldsymbol{U}_{\sigma}^{k-1}\right)\right]$ in $(2.10 \mathrm{a})$, with $L^{\sigma}$ replaced by $\widehat{L}^{\sigma}$, and noting $(2.97 \mathrm{a}),(2.99),(2.96)$ and that $\boldsymbol{U}_{\sigma}^{k-1} \in \boldsymbol{K}_{\boldsymbol{m}}^{h}$ yields

$$
\begin{aligned}
& \left(\frac{\boldsymbol{U}_{\sigma}^{k}-\boldsymbol{U}_{\sigma}^{k-1}}{\tau_{k}}, \boldsymbol{D} F^{\sigma}\left(\boldsymbol{U}_{\sigma}^{k-1}\right)\right)^{h}=-\left(\widehat{L}^{\sigma}\left(\boldsymbol{U}_{\sigma}^{k-1}\right) \nabla \boldsymbol{W}_{\sigma}^{k}, \nabla\left[\boldsymbol{\pi}^{h} \boldsymbol{D} F^{\sigma}\left(\boldsymbol{U}_{\sigma}^{k-1}\right)\right]\right) \\
& =-\sum_{n=1}^{N}\left(\sum_{p=1}^{N}\left[\delta_{n p}-\frac{\widehat{l}_{p}^{\sigma}\left(U_{\sigma, p}^{k-1}\right)}{\mathbf{1}^{T} \widehat{\boldsymbol{l}}^{\sigma}\left(\boldsymbol{U}_{\sigma}^{k-1}\right)}\right] \nabla W_{\sigma, p}^{k}, \widehat{l}_{n}^{\sigma}\left(U_{\sigma, n}^{k-1}\right) \nabla \pi^{h}\left[\left(F_{n}^{\sigma}\right)^{\prime}\left(U_{\sigma, n}^{k-1}\right)\right]\right) \\
& =-\sum_{n=1}^{N}\left(\sum_{p=1}^{N}\left[\delta_{n p}-\frac{\widehat{l}_{p}^{\sigma}\left(U_{\sigma, p}^{k-1}\right)}{\mathbf{1}^{T} \widehat{\boldsymbol{l}}^{\sigma}\left(\boldsymbol{U}_{\sigma}^{k-1}\right)}\right] \nabla W_{\sigma, p}^{k}, \nabla U_{\sigma, n}^{k-1}\right) \\
& =-\sum_{n=1}^{N}\left(\nabla W_{\sigma, n}^{k}, \nabla U_{\sigma, n}^{k-1}\right) .
\end{aligned}
$$

From (2.10b) we have for $n=1 \rightarrow N$ that

$$
\begin{aligned}
& \gamma\left(\nabla U_{\sigma, n}^{k}, \nabla \beta_{j}\right)+\left(\psi_{1}^{\prime}\left(U_{\sigma, n}^{k}\right)-\{\right.\left.\left.A^{-} \boldsymbol{U}_{\sigma}^{k}\right\}_{n}+\left\{A^{+} \boldsymbol{U}_{\sigma}^{k-1}\right\}_{n}, \beta_{j}\right)^{h} \\
& \begin{cases}\geq\left(W_{\sigma, n}^{k}, \beta_{j}\right)^{h} & \forall j \in J, \\
=\left(W_{\sigma, n}^{k}, \beta_{j}\right)^{h} & \text { if } U_{\sigma, n}^{k}\left(x_{j}\right)>0 .\end{cases}
\end{aligned}
$$

From $(2.100),(2.1)$ and (2.101) we have for $n=1 \rightarrow N$ that

$$
\begin{aligned}
U_{\sigma, n}^{k}\left(x_{j}\right)=0 \Longrightarrow\left(1, \beta_{j}\right)^{h}\left(\Delta^{h} U_{\sigma, n}^{k}\right)\left(x_{j}\right) & =\left(\Delta^{h} U_{\sigma, n}^{k}, \beta_{j}\right)^{h}=-\left(\nabla U_{\sigma, n}^{k}, \nabla \beta_{j}\right) \\
& =-\sum_{i \in J} U_{\sigma, n}^{k}\left(x_{i}\right)\left(\nabla \beta_{i}, \nabla \beta_{j}\right) \geq 0 .
\end{aligned}
$$

Combining (2.104) and (2.105) yields for $n=1 \rightarrow N$ that

$$
\gamma\left(\nabla U_{\sigma, n}^{k}, \nabla\left(\Delta^{h} U_{\sigma, n}^{k}\right)\right)+\left(\psi_{1}^{\prime}\left(U_{\sigma, n}^{k}\right)-\left\{A^{-} \boldsymbol{U}_{\sigma}^{k}\right\}_{n}+\left\{A^{+} \boldsymbol{U}_{\sigma}^{k-1}\right\}_{n}, \Delta^{h} U_{\sigma, n}^{k}\right)^{h} \geq\left(W_{\sigma, n}^{k}, \Delta^{h} U_{\sigma, n}^{k}\right)^{h} .
$$


From (2.100), (2.106) and as $\psi_{1} \in C^{1}[0,1]$ and $\boldsymbol{U}_{\sigma}^{k} \in \boldsymbol{K}_{\boldsymbol{m}}^{h}$ we have for $n=1 \rightarrow N$ that

$$
\begin{aligned}
\gamma\left|\Delta^{h} U_{\sigma, n}^{k}\right|_{h}^{2}=-\gamma\left(\nabla U_{\sigma, n}^{k}, \nabla\left(\Delta^{h} U_{\sigma, n}^{k}\right)\right) & \leq-\left(W_{\sigma, n}^{k}, \Delta^{h} U_{\sigma, n}^{k}\right)^{h}+C \\
& =\left(\nabla W_{\sigma, n}^{k}, \nabla U_{\sigma, n}^{k}\right)+C .
\end{aligned}
$$

It follows from (2.103), (2.94a,c), (2.107), $\boldsymbol{U}_{\sigma}^{k}-\boldsymbol{U}_{\sigma}^{k-1} \in \boldsymbol{V}^{h},(2.98)$ and (2.97a) that

$$
\begin{aligned}
& \left(F^{\sigma}\left(\boldsymbol{U}_{\sigma}^{k}\right)-F^{\sigma}\left(\boldsymbol{U}_{\sigma}^{k-1}\right), 1\right)^{h}+\gamma \tau_{k}\left|\boldsymbol{\Delta}^{h} \boldsymbol{U}_{\sigma}^{k}\right|_{h}^{2} \\
& \quad \leq\left(\boldsymbol{U}_{\sigma}^{k}-\boldsymbol{U}_{\sigma}^{k-1}, \boldsymbol{D} F^{\sigma}\left(\boldsymbol{U}_{\sigma}^{k}\right)\right)^{h}+\tau_{k}\left[\left(\nabla \boldsymbol{W}_{\sigma}^{k}, \nabla \boldsymbol{U}_{\sigma}^{k}\right)+C\right] \\
& \quad=\left(\boldsymbol{U}_{\sigma}^{k}-\boldsymbol{U}_{\sigma}^{k-1}, \boldsymbol{D} F^{\sigma}\left(\boldsymbol{U}_{\sigma}^{k}\right)-\boldsymbol{D} F^{\sigma}\left(\boldsymbol{U}_{\sigma}^{k-1}\right)\right)^{h}+\tau_{k}\left[\left(\nabla\left(I-\mathbf{1} \sum\right) \boldsymbol{W}_{\sigma}^{k}, \nabla\left(\boldsymbol{U}_{\sigma}^{k}-\boldsymbol{U}_{\sigma}^{k-1}\right)\right)+C\right] \\
& \quad \leq C \sigma^{-1} \tau_{k}^{2}\left|\frac{\boldsymbol{U}_{\sigma}^{k}-\boldsymbol{U}_{\sigma}^{k-1}}{\tau_{k}}\right|_{h}^{2}+C \sigma^{-1} \tau_{k}^{2}\left|\left[\widehat{L}^{\sigma}\left(\boldsymbol{U}_{\sigma}^{k-1}\right)\right]^{\frac{1}{2}} \nabla \boldsymbol{W}_{\sigma}^{k}\right|_{0}\left|\frac{\boldsymbol{U}_{\sigma}^{k}-\boldsymbol{U}_{\sigma}^{k-1}}{\tau_{k}}\right|_{1}+C \tau_{k} .
\end{aligned}
$$

Next we note from $(2.27)$ and the analogue of $(2.30)$ for $\widehat{L}^{\sigma}(\cdot)$ that

$$
\begin{aligned}
\sum_{k=1}^{K} \tau_{k}^{2}\left|\frac{\boldsymbol{U}_{\sigma}^{k}-\boldsymbol{U}_{\sigma}^{k-1}}{\tau_{k}}\right|_{h}^{2} & \leq\left[\sum_{k=1}^{K} \tau_{k}^{2}\left|\frac{\boldsymbol{U}_{\sigma}^{k}-\boldsymbol{U}_{\sigma}^{k-1}}{\tau_{k}}\right|_{1}^{2}\right]^{\frac{1}{2}}\left[\sum_{k=1}^{K} \tau_{k}^{2}\left|\hat{\mathcal{G}}^{h}\left[\frac{\boldsymbol{U}_{\sigma}^{k}-\boldsymbol{U}_{\sigma}^{k-1}}{\tau_{k}}\right]\right|_{1}^{2}\right]^{\frac{1}{2}} \\
& \leq C \tau^{\frac{1}{2}}\left[L_{\max }^{\sigma}\right]^{\frac{1}{2}}\left[\left|\boldsymbol{U}_{\sigma}^{0}\right|_{1}^{2}+1\right] .
\end{aligned}
$$

Summing (2.108) from $k=1 \rightarrow m$, for $m=1 \rightarrow K$, and noting (2.30), with $L^{\sigma}$ replaced by $\widehat{L}^{\sigma}$, and (2.109) yields the desired result (2.102).

Finally it follows from (2.102), $\tau \leq C \sigma^{2}$, assumption i) of Theorem 2.2, (2.94b) and (2.50b) that

$$
\left\|\boldsymbol{\Delta}^{h} \boldsymbol{U}_{\sigma}^{+}\right\|_{\boldsymbol{L}^{2}\left(\Omega_{T}\right)}^{2} \leq C+\left(F^{\sigma}\left(\boldsymbol{U}_{\sigma}^{0}\right), 1\right)^{h} \leq C .
$$

From (2.110) it follows that there exists a $\boldsymbol{\Gamma} \in \boldsymbol{L}^{2}\left(\Omega_{T}\right)$ such that

$$
\boldsymbol{\Delta}^{h} \boldsymbol{U}_{\sigma}^{+} \rightarrow \boldsymbol{\Gamma} \quad \text { weakly in } \boldsymbol{L}^{2}\left(\Omega_{T}\right) \text { as } h \rightarrow 0 .
$$

It follows (2.100) with $\boldsymbol{\chi} \equiv \boldsymbol{\pi}^{h} \boldsymbol{\eta},(2.85),(2.16),(2.17)$ and (2.111) that

$$
\int_{0}^{T}\left(\frac{\partial}{\partial x} \boldsymbol{u}, \frac{\partial}{\partial x} \boldsymbol{\eta}\right) \mathrm{d} t=-\int_{0}^{T}(\boldsymbol{\Gamma}, \boldsymbol{\eta}) \mathrm{d} t \quad \forall \boldsymbol{\eta} \in L^{2}\left(0, T ; \boldsymbol{H}^{1}(\Omega)\right) .
$$

The desired result $\boldsymbol{u} \in L^{2}\left(0, T ; \boldsymbol{H}^{2}(\Omega)\right)$ then follows from (2.112) as $\boldsymbol{\Gamma} \in \boldsymbol{L}^{2}\left(\Omega_{T}\right)$.

Remark 2.2. Under the assumptions of Theorem 2.2, except that $d \geq 2$, it still follows from (2.58) and (2.59) that there exists a vector function $\boldsymbol{u} \in L^{\infty}\left(0, T ; \boldsymbol{K}_{\boldsymbol{m}}\right) \cap H^{1}\left(0, T ;\left(\boldsymbol{H}^{1}(\Omega)\right)^{\prime}\right)$ such that as $h \rightarrow 0$

$$
\begin{aligned}
& \boldsymbol{U}_{(\sigma)}, \boldsymbol{U}_{(\sigma)}^{ \pm} \rightarrow \boldsymbol{u} \quad \text { weakly in } \quad L^{2}\left(0, T ; \boldsymbol{H}^{1}(\Omega)\right) \cap H^{1}\left(0, T ;\left(\boldsymbol{H}^{1}(\Omega)\right)^{\prime}\right) \\
& \boldsymbol{U}_{(\sigma)}, \boldsymbol{U}_{(\sigma)}^{ \pm} \rightarrow \boldsymbol{u} \text { strongly in } L^{2}\left(\Omega_{T}\right) .
\end{aligned}
$$

Moreover, with the mobility matrix $L^{\sigma}$ replaced by $\widehat{L}^{\sigma}$ and under the additional assumptions $(\widetilde{\mathrm{A}})$ and the additional constraint $\tau \leq C \sigma^{2}$ as $h \rightarrow 0$ we have that (2.113) still hold for $\boldsymbol{U}_{\sigma}^{( \pm)}$with $\boldsymbol{u} \in L^{2}\left(0, T ; \boldsymbol{H}^{2}(\Omega)\right)$ if $d \geq 2$, see Theorem 2.3 above. However, like Grün and Rumpf [24], we are not able to show that $\boldsymbol{u}$ solves a weak form of $(\mathrm{P})$ if $d \geq 2$.

We end this section by noting that in Barrett and Blowey [6] an optimal error bound is proved for the approximation $\left(\mathrm{P}^{h, \tau}\right)$ in the case of a non-degenerate mobility matrix with strengthened assumptions on the initial data $\boldsymbol{u}^{0}$. 


\section{Solution of the Discrete VARIATional InEQUALity}

We now consider an algorithm for solving the variational inequality system at each time level in $\left(\mathrm{P}^{h, \tau}\right)$. This is a multi-component version of the algorithm introduced in Barrett et al. [8, Sect. 3] for the scalar Cahn-Hilliard problem with degenerate mobility, which is based on the general splitting algorithm of Lions and Mercier [26].

Throughout this section, we adopt the notation:

$$
\bar{\chi}:=\left(I-1 \sum\right) \chi \quad \forall \chi \in S^{h} .
$$

Then for $k$ fixed, multiplying (2.10b), (2.35b) by $\mu>0$, adding $\left(\boldsymbol{U}_{(\sigma)}^{k}, \boldsymbol{\chi}-\boldsymbol{U}_{(\sigma)}^{k}\right)^{h}$ to both sides and rearranging on noting (2.10a), (2.35a); it follows that $\left\{\boldsymbol{U}_{(\sigma)}^{k}, \boldsymbol{W}_{(\sigma)}^{k}\right\} \in \boldsymbol{K}_{\boldsymbol{m}}^{h} \times \boldsymbol{S}^{h}$ satisfy

$$
\begin{aligned}
\left(\boldsymbol{U}_{(\sigma)}^{k}+\mu \boldsymbol{D} \Psi_{1}\left(\boldsymbol{U}_{(\sigma)}^{k}\right), \boldsymbol{\chi}-\boldsymbol{U}_{(\sigma)}^{k}\right)^{h} & \geq\left(\boldsymbol{R}^{k}, \boldsymbol{\chi}-\boldsymbol{U}_{(\sigma)}^{k}\right)^{h} \quad \forall \boldsymbol{\chi} \in \boldsymbol{K}^{h}, \\
\left(\frac{\boldsymbol{U}_{(\sigma)}^{k}-\boldsymbol{U}_{(\sigma)}^{k-1}}{\tau_{k}}, \boldsymbol{\chi}\right)^{h}+L_{\max }^{(\sigma)}\left(\nabla \overline{\boldsymbol{W}}_{(\sigma)}^{k}, \nabla \boldsymbol{\chi}\right) & =\left(\left[L_{\max }^{(\sigma)} I-L^{(\sigma)}\left(\boldsymbol{U}_{(\sigma)}^{k-1}\right)\right] \nabla \overline{\boldsymbol{W}}_{(\sigma)}^{k}, \nabla \boldsymbol{\chi}\right) \quad \forall \boldsymbol{\chi} \in \boldsymbol{S}^{h} ;
\end{aligned}
$$

where $\boldsymbol{R}^{k} \in \boldsymbol{S}^{h}$ is such that

$$
\left(\boldsymbol{R}^{k}-\boldsymbol{U}_{(\sigma)}^{k}, \boldsymbol{\chi}\right)^{h}:=-\mu\left[\gamma\left(\nabla \boldsymbol{U}_{(\sigma)}^{k}, \nabla \boldsymbol{\chi}\right)-\left(A^{-} \boldsymbol{U}_{(\sigma)}^{k}+A^{+} \boldsymbol{U}_{(\sigma)}^{k-1}+\boldsymbol{W}_{(\sigma)}^{k}, \boldsymbol{\chi}\right)^{h}\right] \quad \forall \boldsymbol{\chi} \in \boldsymbol{S}^{h}
$$

and $L_{\max }^{(\sigma)} \in \mathbb{R}$ is as defined in (2.9). We introduce also $\boldsymbol{X}^{k} \in \boldsymbol{S}^{h}$ such that

$$
\left(\boldsymbol{X}^{k}-\boldsymbol{U}_{(\sigma)}^{k}, \boldsymbol{\chi}\right)^{h}:=\mu\left[\gamma\left(\nabla \boldsymbol{U}_{(\sigma)}^{k}, \nabla \boldsymbol{\chi}\right)-\left(A^{-} \boldsymbol{U}_{(\sigma)}^{k}+A^{+} \boldsymbol{U}_{(\sigma)}^{k-1}+\boldsymbol{W}_{(\sigma)}^{k}, \boldsymbol{\chi}\right)^{h}\right] \quad \forall \boldsymbol{\chi} \in \boldsymbol{S}^{h}
$$

and note that $\boldsymbol{X}^{k}=2 \boldsymbol{U}_{(\sigma)}^{k}-\boldsymbol{R}^{k}$. We use the above as a basis for constructing our iterative procedure:

For $k \geq 1$ set $\left\{\boldsymbol{U}^{k, 0}, \boldsymbol{W}^{k, 0}\right\} \equiv\left\{\boldsymbol{U}_{(\sigma)}^{k-1}, \boldsymbol{W}_{(\sigma)}^{k-1}\right\} \in \boldsymbol{K}_{\boldsymbol{m}}^{h} \times \boldsymbol{S}^{h}$, where $\boldsymbol{W}_{(\sigma)}^{0} \in \boldsymbol{S}^{h}$ is arbitrary if $k=1$.

For $p \geq 0$ we define $\boldsymbol{R}^{k, p} \in \boldsymbol{S}^{h}$ such that

$$
\left(\boldsymbol{R}^{k, p}-\boldsymbol{U}^{k, p}, \boldsymbol{\chi}\right)^{h}=-\mu\left[\gamma\left(\nabla \boldsymbol{U}^{k, p}, \nabla \boldsymbol{\chi}\right)-\left(A^{-} \boldsymbol{U}^{k, p}+A^{+} \boldsymbol{U}_{(\sigma)}^{k-1}+\boldsymbol{W}^{k, p}, \boldsymbol{\chi}\right)^{h}\right] \quad \forall \boldsymbol{\chi} \in \boldsymbol{S}^{h} .
$$

Then, on recalling the notation (2.36), find $\boldsymbol{U}^{k, p+\frac{1}{2}} \in \boldsymbol{K}^{h}$ such that for $n=1 \rightarrow N$

$$
\begin{gathered}
U_{n}^{k, p+\frac{1}{2}}\left(\boldsymbol{x}_{j}\right)=U_{(\sigma,) n}^{k-1}\left(\boldsymbol{x}_{j}\right) \quad \text { if } j \in J_{0}\left(U_{(\sigma,) n}^{k-1}\right) \\
\left(U_{n}^{k, p+\frac{1}{2}}\left(\boldsymbol{x}_{j}\right)+\mu \psi_{1}^{\prime}\left(U_{n}^{k, p+\frac{1}{2}}\left(\boldsymbol{x}_{j}\right)\right)-R_{n}^{k, p}\left(\boldsymbol{x}_{j}\right)\right)\left(r-U_{n}^{k, p+\frac{1}{2}}\left(\boldsymbol{x}_{j}\right)\right) \geq 0 \quad \forall r \geq 0 \quad \text { if } j \in J_{+}\left(U_{(\sigma,) n}^{k-1}\right)
\end{gathered}
$$

and find $\left\{\boldsymbol{U}^{k, p+1}, \boldsymbol{W}^{k, p+1}\right\} \in \boldsymbol{S}^{h} \times \boldsymbol{S}^{h}$ such that

$$
\begin{gathered}
\left(\frac{\boldsymbol{U}^{k, p+1}-\boldsymbol{U}_{(\sigma)}^{k-1}}{\tau_{k}}, \boldsymbol{\chi}\right)^{h}+L_{\max }^{(\sigma)}\left(\nabla \overline{\boldsymbol{W}}^{k, p+1}, \nabla \boldsymbol{\chi}\right)=\left(\left[L_{\max }^{(\sigma)} I-L^{(\sigma)}\left(\boldsymbol{U}_{(\sigma)}^{k-1}\right)\right] \nabla \overline{\boldsymbol{W}}^{k, p}, \nabla \boldsymbol{\chi}\right) \quad \forall \boldsymbol{\chi} \in \boldsymbol{S}^{h}, \quad(3.3 \mathrm{c}) \\
\left(\boldsymbol{U}^{k, p+1}, \boldsymbol{\chi}\right)^{h}+\mu\left[\gamma\left(\nabla \boldsymbol{U}^{k, p+1}, \nabla \boldsymbol{\chi}\right)-\left(A^{-} \boldsymbol{U}^{k, p+1}+\boldsymbol{W}^{k, p+1}, \boldsymbol{\chi}\right)^{h}\right]=\left(\boldsymbol{X}^{k, p+1}+\mu A^{+} \boldsymbol{U}_{(\sigma)}^{k-1}, \boldsymbol{\chi}\right)^{h} \quad \forall \boldsymbol{\chi} \in \boldsymbol{S}^{h} ;
\end{gathered}
$$

where $\boldsymbol{X}^{k, p+1}:=2 \boldsymbol{U}^{k, p+\frac{1}{2}}-\boldsymbol{R}^{k, p}$. For $j \in J_{+}\left(U_{(\sigma,) n}^{k-1}\right)$ existence and uniqueness of $U_{n}^{k, j+\frac{1}{2}}\left(\boldsymbol{x}_{j}\right)$ in the variational inequality (3.3b) follows from the monotonicity of $\psi_{1}^{\prime}(\cdot)$. 
It remains to show that there exists a unique solution to (3.3c,d). On noting (1.14a) and (2.5a), let $\boldsymbol{E}^{k, p} \in \boldsymbol{V}^{h}$ be such that

$$
\left(\boldsymbol{E}^{k, p}, \boldsymbol{\chi}\right)^{h}:=\left(L^{(\sigma)}\left(\boldsymbol{U}_{(\sigma)}^{k-1}\right) \nabla \boldsymbol{W}^{k, p}, \nabla \boldsymbol{\chi}\right) \equiv\left(L^{(\sigma)}\left(\boldsymbol{U}_{(\sigma)}^{k-1}\right) \nabla \overline{\boldsymbol{W}}^{k, p}, \nabla \boldsymbol{\chi}\right) \quad \forall \boldsymbol{\chi} \in \boldsymbol{S}^{h} .
$$

It then follows from $(3.3 \mathrm{c}, \mathrm{d})$ and $(2.22)$ that

$$
\begin{aligned}
\sum \boldsymbol{W}^{k, p+1}= & \mu^{-1}\left(N^{-1}-\sum \boldsymbol{X}^{k, p+1}\right)-\sum\left(A^{-} \boldsymbol{U}^{k, p+1}+A^{+} \boldsymbol{U}_{(\sigma)}^{k-1}\right), \\
\overline{\boldsymbol{W}}^{k, p+1}= & (I-f) \overline{\boldsymbol{W}}^{k, p}-\left[L_{\max }^{(\sigma)}\right]^{-1} \hat{\mathcal{G}}^{h}\left(\frac{\boldsymbol{U}^{k, p+1}-\boldsymbol{U}_{(\sigma)}^{k-1}}{\tau_{k}}+\boldsymbol{E}^{k, p}\right) \\
& +f\left[\mu^{-1}\left(\boldsymbol{U}^{k, p+1}-\boldsymbol{X}^{k, p+1}\right)-A \boldsymbol{m}-\sum \boldsymbol{W}^{k, p+1} \mathbf{1}\right] .
\end{aligned}
$$

Therefore (3.3c,d) may be written equivalently as:

Find $\boldsymbol{U}^{k, p+1} \in \boldsymbol{S}_{\boldsymbol{m}}^{h}:=\left\{\boldsymbol{v}^{h} \in \boldsymbol{S}^{h}: f \boldsymbol{v}^{h}=f \boldsymbol{U}^{0}, N \sum \boldsymbol{v}^{h}=1\right\}$ such that

$$
\begin{aligned}
& \left(\boldsymbol{U}^{k, p+1},(I-f) \overline{\boldsymbol{\chi}}\right)^{h}+\mu\left[\gamma\left(\nabla \boldsymbol{U}^{k, p+1}, \nabla \boldsymbol{\chi}\right)+\left(\left[L_{\max }^{(\sigma)}\right]^{-1} \hat{\mathcal{G}}^{h}\left(\frac{\boldsymbol{U}^{k, p+1}-\boldsymbol{U}_{(\sigma)}^{k-1}}{\tau_{k}}\right)-A^{-} \boldsymbol{U}^{k, p+1},(I-f) \overline{\boldsymbol{\chi}}\right)^{h}\right] \\
& =\left(\boldsymbol{B}^{k, p+1},(I-f) \bar{\chi}\right)^{h} \quad \forall \chi \in \boldsymbol{S}^{h},
\end{aligned}
$$

Existence and uniqueness of $\boldsymbol{U}^{k, p+1} \in \boldsymbol{S}_{\boldsymbol{m}}^{h}$ satisfying (3.6a) follows since this is the Euler-Lagrange equation of the strictly convex minimization problem

$$
\min _{\boldsymbol{\chi} \in \boldsymbol{S}_{\boldsymbol{m}}^{h}}\left\{|\boldsymbol{\chi}|_{h}^{2}+\mu\left[\gamma|\boldsymbol{\chi}|_{1}^{2}+\frac{1}{L_{\max }^{(\sigma)} \tau_{k}}\left|\nabla \hat{\mathcal{G}}^{h}\left(\boldsymbol{\chi}-\boldsymbol{U}_{(\sigma)}^{k-1}\right)\right|_{0}^{2}-\left(A^{-} \boldsymbol{\chi}, \boldsymbol{\chi}\right)^{h}\right]-2\left(\boldsymbol{B}^{k, p+1}, \boldsymbol{\chi}\right)^{h}\right\}
$$

Finally, existence and uniqueness of $\boldsymbol{W}^{k, p+1}$ then follows from (3.5a,b). Hence the iterative procedure (3.3a-d) is well-defined. Moreover, at each iteration one needs to solve only (i) a fixed linear system with constant coefficients and (ii) a decoupled nonlinear equation for each component at each mesh point. We briefly discuss the solution of (i); that is, (3.6a). Introducing the $\mathcal{J} \times N$ matrices $\mathcal{U}^{k, p} \equiv\left\{\mathcal{U}_{j, n}^{k, p}\right\}$ and $\mathcal{B}^{k, p} \equiv\left\{\mathcal{B}_{j, n}^{k, p}\right\}$ where $\mathcal{J}:=\# J$ and

$$
\left\{\boldsymbol{U}^{k, p}\right\}_{n}(\boldsymbol{x}) \equiv \sum_{j=1}^{\mathcal{J}} \mathcal{U}_{j, n}^{k, p} \beta_{j}(\boldsymbol{x}), \quad\left\{\boldsymbol{B}^{k, p}\right\}_{n}(\boldsymbol{x}) \equiv \sum_{j=1}^{\mathcal{J}} \mathcal{B}_{j, n}^{k, p} \beta_{j}(\boldsymbol{x})
$$

In addition, we introduce the $N \times N$ matrix $L^{*}:=I-\frac{1}{N} \mathbf{1} \mathbf{1}^{T}$ and the $\mathcal{J} \times \mathcal{J}$ matrices $\mathcal{K} \equiv\left\{\mathcal{K}_{j, j^{\prime}}\right\}$ and $\mathcal{D} \equiv\left\{\mathcal{D}_{j, j^{\prime}}\right\}$, where

$$
\mathcal{K}_{j, j^{\prime}}:=\left(\nabla \beta_{j}, \nabla \beta_{j^{\prime}}\right), \quad \mathcal{D}_{j, j^{\prime}}:=\left(\beta_{j}, \beta_{j^{\prime}}\right)^{h}=\omega_{j} \delta_{j j^{\prime}} .
$$

Using this notation, setting $\mathcal{M} \equiv \mathcal{D}^{-1} \mathcal{K}$, noting that $\left(L^{*}\right)^{T} \equiv L^{*}$ and $L^{*} \mathbf{1}=\mathbf{0}$, (3.6a) may be restated as find $\mathcal{U}^{k, p+1} \in \mathbb{R}^{\mathcal{J}, N}$ such that

$$
\mathcal{M} \mathcal{U}^{k, p+1} L^{*}+\mu\left[\gamma \mathcal{M}^{2} \mathcal{U}^{k, p+1} L^{*}+\frac{\left[L_{\max }^{(\sigma)}\right]^{-1}}{\tau_{k}}\left(\mathcal{U}^{k, p+1}-\mathcal{U}^{k-1}\right)-\mathcal{M} \mathcal{U}^{k, p+1} A^{-} L^{*}\right]=\mathcal{M} B^{k, p+1} L^{*}
$$

Systems of this type are easily solved using "the discrete cosine transform" when $\mathcal{T}^{h}$ is a uniform partitioning, see Barrett and Blowey [3, Sect. 4] for details. 
Theorem 3.1. For all $\mu \in \mathbb{R}^{+}$and $\left\{\boldsymbol{U}^{k, 0}, \boldsymbol{W}^{k, 0}\right\} \in \boldsymbol{K}_{m}^{h} \times \boldsymbol{S}^{h}$ the sequence $\left\{\boldsymbol{U}^{k, p}, \boldsymbol{W}^{k, p}\right\}_{p \geq 0}$ generated by the algorithm (3.3a-d) satisfies

$$
\boldsymbol{U}^{k, p} \rightarrow \boldsymbol{U}_{(\sigma)}^{k} \quad \text { and } \quad \int_{\Omega} L^{(\sigma)}\left(\boldsymbol{U}_{(\sigma)}^{k-1}\right)\left|\nabla\left(\boldsymbol{W}^{k, p+1}-\boldsymbol{W}_{(\sigma)}^{k}\right)\right|^{2} \mathrm{~d} x \rightarrow 0 \quad \text { as } \quad p \rightarrow \infty .
$$

In addition, if $\psi_{1}^{\prime}(\cdot)$ is strictly monotone then $\boldsymbol{U}^{k, p+\frac{1}{2}} \rightarrow \boldsymbol{U}_{(\sigma)}^{k}$ as $p \rightarrow \infty$.

Proof. It follows from $(3.2 \mathrm{c}, \mathrm{d}),(3.3 \mathrm{a}, \mathrm{d})$ and by the definition of $\boldsymbol{X}^{k, p+1}$ that for $p \geq 0$

$$
\boldsymbol{U}_{(\sigma)}^{k}=\frac{1}{2}\left(\boldsymbol{X}^{k}+\boldsymbol{R}^{k}\right), \quad \boldsymbol{U}^{k, p}=\frac{1}{2}\left(\boldsymbol{X}^{k, p}+\boldsymbol{R}^{k, p}\right), \quad \boldsymbol{U}^{k, p+\frac{1}{2}}=\frac{1}{2}\left(\boldsymbol{X}^{k, p+1}+\boldsymbol{R}^{k, p}\right) .
$$

It follows from (3.3d), (3.2d) and (3.11) that

$$
\begin{aligned}
\gamma\left|\boldsymbol{U}^{k, p+1}-\boldsymbol{U}_{(\sigma)}^{k}\right|_{1}^{2}-\left(A^{-}\left(\boldsymbol{U}^{k, p+1}-\boldsymbol{U}_{(\sigma)}^{k}\right)-\left(\boldsymbol{W}^{k, p+1}-\boldsymbol{W}_{(\sigma)}^{k}\right), \boldsymbol{U}^{k, p+1}-\boldsymbol{U}_{(\sigma)}^{k}\right)^{h} \\
\quad=\frac{1}{4 \mu}\left(\boldsymbol{X}^{k, p+1}-\boldsymbol{X}^{k}-\boldsymbol{R}^{k, p+1}+\boldsymbol{R}^{k}, \boldsymbol{X}^{k, p+1}-\boldsymbol{X}^{k}+\boldsymbol{R}^{k, p+1}-\boldsymbol{R}^{k}\right)^{h} \\
=\frac{1}{4 \mu}\left(\left|\boldsymbol{X}^{k, p+1}-\boldsymbol{X}^{k}\right|_{h}^{2}-\left|\boldsymbol{R}^{k, p+1}-\boldsymbol{R}^{k}\right|_{h}^{2}\right) .
\end{aligned}
$$

Choosing $\chi \equiv \boldsymbol{U}^{k, p+\frac{1}{2}}$ in (3.2a) and for $j \in J_{+}\left(U_{(\sigma,) n}^{k-1}\right)$ choosing $r \equiv U_{(\sigma,) n}^{k}\left(\boldsymbol{x}_{j}\right)$ in (3.3b), multiplying by $\omega_{j}$ on recalling (2.1) and summing over $n$ and $j$, yields on noting that $U_{(\sigma,) n}^{k}\left(\boldsymbol{x}_{j}\right)=U^{k, p+\frac{1}{2}}\left(\boldsymbol{x}_{j}\right)$ for $j \in J_{0}\left(U_{(\sigma,) n}^{k-1}\right)$

$$
\left|\boldsymbol{U}^{k, p+\frac{1}{2}}-\boldsymbol{U}_{(\sigma)}^{k}\right|_{h}^{2}+\mu\left(\boldsymbol{D} \Psi_{1}\left(\boldsymbol{U}^{k, p+\frac{1}{2}}\right)-\boldsymbol{D} \Psi_{1}\left(\boldsymbol{U}_{(\sigma)}^{k}\right), \boldsymbol{U}^{k, p+\frac{1}{2}}-\boldsymbol{U}_{(\sigma)}^{k}\right)^{h} \leq\left(\boldsymbol{R}^{k, p}-\boldsymbol{R}^{k}, \boldsymbol{U}^{k, p+\frac{1}{2}}-\boldsymbol{U}_{(\sigma)}^{k}\right)^{h}
$$

Combining (3.13) and (3.11) yields that

$$
4 \mu\left(\boldsymbol{D} \Psi_{1}\left(\boldsymbol{U}^{k, p+\frac{1}{2}}\right)-\boldsymbol{D} \Psi_{1}\left(\boldsymbol{U}_{(\sigma)}^{k}\right), \boldsymbol{U}^{k, p+\frac{1}{2}}-\boldsymbol{U}_{(\sigma)}^{k}\right)^{h}+\left|\boldsymbol{X}^{k, p+1}-\boldsymbol{X}^{k}\right|_{h}^{2} \leq\left|\boldsymbol{R}^{k, p}-\boldsymbol{R}^{k}\right|_{h}^{2} .
$$

As $\boldsymbol{U}^{k, p+1}, \boldsymbol{U}_{(\sigma)}^{k} \in \boldsymbol{S}_{\boldsymbol{m}}^{h}$, using (3.3c), (3.2b), (1.14a), (2.5a) and (2.33) it follows that

$$
\begin{aligned}
-\left(\boldsymbol{W}^{k, p+1}-\boldsymbol{W}_{(\sigma)}^{k},\right. & \left.\boldsymbol{U}^{k, p+1}-\boldsymbol{U}_{(\sigma)}^{k}\right)^{h} \\
= & -\left(\overline{\boldsymbol{W}}^{k, p+1}-\overline{\boldsymbol{W}}_{(\sigma)}^{k}, \boldsymbol{U}^{k, p+1}-\boldsymbol{U}_{(\sigma)}^{k-1}\right)^{h}-\left(\overline{\boldsymbol{W}}^{k, p+1}-\overline{\boldsymbol{W}}_{(\sigma)}^{k}, \boldsymbol{U}_{(\sigma)}^{k-1}-\boldsymbol{U}_{(\sigma)}^{k}\right)^{h} \\
= & \tau_{k}\left|\left[L^{(\sigma)}\left(\boldsymbol{U}_{(\sigma)}^{k-1}\right)\right]^{\frac{1}{2}} \nabla\left(\boldsymbol{W}^{k, p+1}-\boldsymbol{W}_{(\sigma)}^{k}\right)\right|_{0}^{2} \\
& +\tau_{k}\left(\left[L_{\max }^{(\sigma)} I-L^{(\sigma)}\left(\boldsymbol{U}_{(\sigma)}^{k-1}\right)\right] \nabla\left(\overline{\boldsymbol{W}}^{k, p+1}-\overline{\boldsymbol{W}}^{k, p}\right), \nabla\left(\overline{\boldsymbol{W}}^{k, p+1}-\overline{\boldsymbol{W}}_{(\sigma)}^{k}\right)\right) \\
= & \tau_{k}\left|\left[L^{(\sigma)}\left(\boldsymbol{U}_{(\sigma)}^{k-1}\right)\right]^{\frac{1}{2}} \nabla\left(\boldsymbol{W}^{k, p+1}-\boldsymbol{W}_{(\sigma)}^{k}\right)\right|_{0}^{2} \\
& +\frac{\tau_{k}}{2}\left[\left|\left[L_{\max }^{(\sigma)} I-L^{(\sigma)}\left(\boldsymbol{U}_{(\sigma)}^{k-1}\right)\right]^{\frac{1}{2}} \nabla\left(\overline{\boldsymbol{W}}^{k, p+1}-\overline{\boldsymbol{W}}_{(\sigma)}^{k}\right)\right|_{0}^{2}\right. \\
& -\left|\left[L_{\max }^{(\sigma)} I-L^{(\sigma)}\left(\boldsymbol{U}_{(\sigma)}^{k-1}\right)\right]^{\frac{1}{2}} \nabla\left(\overline{\boldsymbol{W}}^{k, p}-\overline{\boldsymbol{W}}_{(\sigma)}^{k}\right)\right|_{0}^{2} \\
& \left.+\left|\left[L_{\max }^{(\sigma)} I-L^{(\sigma)}\left(\boldsymbol{U}_{(\sigma)}^{k-1}\right)\right]^{\frac{1}{2}} \nabla\left(\overline{\boldsymbol{W}}^{k, p+1}-\overline{\boldsymbol{W}}^{k, p}\right)\right|_{0}^{2}\right] .
\end{aligned}
$$


Combining (3.12), (3.14), (3.15) and rearranging yields that

$$
\begin{aligned}
& \gamma\left|\boldsymbol{U}^{k, p+1}-\boldsymbol{U}_{(\sigma)}^{k}\right|_{1}^{2}-\left(A^{-}\left(\boldsymbol{U}^{k, p+1}-\boldsymbol{U}_{(\sigma)}^{k}\right), \boldsymbol{U}^{k, p+1}-\boldsymbol{U}_{(\sigma)}^{k}\right)^{h} \\
& \quad+\left(\boldsymbol{D} \Psi_{1}\left(\boldsymbol{U}^{k, p+\frac{1}{2}}\right)-\boldsymbol{D} \Psi_{1}\left(\boldsymbol{U}_{(\sigma)}^{k}\right), \boldsymbol{U}^{k, p+\frac{1}{2}}-\boldsymbol{U}_{(\sigma)}^{k}\right)^{h} \\
& \quad+\tau_{k}\left|\left[L^{(\sigma)}\left(\boldsymbol{U}_{(\sigma)}^{k-1}\right)\right]^{\frac{1}{2}} \nabla\left(\boldsymbol{W}^{k, p+1}-\boldsymbol{W}_{(\sigma)}^{k}\right)\right|_{0}^{2} \\
& \quad+\frac{1}{4 \mu}\left|\boldsymbol{R}^{k, p+1}-\boldsymbol{R}^{k}\right|_{h}^{2}+\frac{\tau_{k}}{2}\left|\left[L_{\max }^{(\sigma)} I-L^{(\sigma)}\left(\boldsymbol{U}_{(\sigma)}^{k-1}\right)\right]^{\frac{1}{2}} \nabla\left(\overline{\boldsymbol{W}}^{k, p+1}-\overline{\boldsymbol{W}}_{(\sigma)}^{k}\right)\right|_{0}^{2} \\
& \quad \leq \frac{1}{4 \mu}\left|\boldsymbol{R}^{k, p}-\boldsymbol{R}^{k}\right|_{h}^{2}+\frac{\tau_{k}}{2}\left|\left[L_{\max }^{(\sigma)} I-L^{(\sigma)}\left(\boldsymbol{U}_{(\sigma)}^{k-1}\right)\right]^{\frac{1}{2}} \nabla\left(\overline{\boldsymbol{W}}^{k, p}-\overline{\boldsymbol{W}}_{(\sigma)}^{k}\right)\right|_{0}^{2} .
\end{aligned}
$$

Therefore noting the monotonicity of $\psi_{1}^{\prime}(\cdot)$ and the negative definiteness of $A^{-}$we have that $\left\{\frac{1}{4 \mu}\left|\boldsymbol{R}^{k, p}-\boldsymbol{R}^{k}\right|_{h}^{2}+\right.$ $\left.\frac{\tau_{k}}{2}\left|\left[L_{\max }^{(\sigma)} I-L^{(\sigma)}\left(\boldsymbol{U}_{(\sigma)}^{k-1}\right)\right]^{\frac{1}{2}} \nabla\left(\overline{\boldsymbol{W}}^{k, p}-\overline{\boldsymbol{W}}_{(\sigma)}^{k}\right)\right|_{0}^{2}\right\}_{p \geq 0}$ is a decreasing sequence which is bounded below and so has a limit. Therefore the desired results (3.10) follow from this and (3.16).

Remark 3.1. Obviously the results above apply also to the approximation $\left(\mathrm{P}_{\sigma}^{h, \tau}\right)$ with the mobility matrix $L^{\sigma}$ replaced by $\widehat{L}^{\sigma}$.

\section{LOGARITHMIC FREE ENERGY}

In this section we modify our approximations $\left(\mathrm{P}_{(\sigma)}^{h, \tau}\right)$ and the results in the previous two sections to cope with the logarithmic free energy, that is

$$
\psi_{1}(r):=\theta r \ln r
$$

Here we have the additional difficulty that $\psi_{1}^{\prime}(\cdot)$ is not uniformly bounded on $(0,1]$ with $\psi_{1}^{\prime}(0)=-\infty$.

Our modified approximations are:

$\left(\widetilde{\mathbf{P}}_{(\sigma)}^{h, \tau}\right)$ For $k \geq 1$, find $\left\{\boldsymbol{U}_{(\sigma)}^{k}, \boldsymbol{W}_{(\sigma)}^{k}\right\} \in \boldsymbol{S}^{h} \times \boldsymbol{S}^{h}$ such that

$$
\begin{gathered}
\left(\frac{\boldsymbol{U}_{(\sigma)}^{k}-\boldsymbol{U}_{(\sigma)}^{k-1}}{\tau_{k}}, \boldsymbol{\chi}\right)^{h}+\left(L^{(\sigma)}\left(\boldsymbol{U}_{(\sigma)}^{k-1}\right) \nabla \boldsymbol{W}_{(\sigma)}^{k}, \nabla \boldsymbol{\chi}\right)=0 \quad \forall \boldsymbol{\chi} \in \boldsymbol{S}^{h}, \\
\gamma\left(\nabla \boldsymbol{U}_{(\sigma)}^{k}, \nabla \boldsymbol{\chi}\right)+\left(\boldsymbol{D} \Psi_{1}\left(\boldsymbol{U}_{(\sigma)}^{k}\right)-A^{-} \boldsymbol{U}_{(\sigma)}^{k}+\boldsymbol{W}_{(\sigma)}^{k}, \boldsymbol{\chi}\right)^{h}=\left(A^{+} \boldsymbol{U}_{(\sigma)}^{k-1}, \boldsymbol{\chi}\right)^{h} \quad \forall \boldsymbol{\chi} \in \widetilde{\boldsymbol{V}}^{h}\left(\boldsymbol{U}_{(\sigma)}^{k-1}\right) ;
\end{gathered}
$$

where $\boldsymbol{U}_{(\sigma)}^{0} \in \boldsymbol{K}_{\boldsymbol{m}}^{h}$ is an approximation of $\boldsymbol{u}^{0} \in \boldsymbol{K}_{\boldsymbol{m}}$ and for all $\boldsymbol{q}^{h} \in \boldsymbol{K}_{\boldsymbol{m}}^{h}$ we define

$$
\widetilde{\boldsymbol{V}}^{h}\left(\boldsymbol{q}^{h}\right):=\left\{\boldsymbol{v}^{h} \in \boldsymbol{S}^{h}: v_{n}^{h}\left(\boldsymbol{x}_{j}\right)=0 \quad \forall j \in J_{0}\left(q_{n}^{h}\right), \quad n=1 \rightarrow N\right\} .
$$

Clearly (4.2b) implicitly implies that $U_{(\sigma,) n}^{k}\left(\boldsymbol{x}_{j}\right)>0$ for all $j \in J_{+}\left(U_{(\sigma,) n}^{k-1}\right), n=1 \rightarrow N$. Unfortunately as with $\left(\mathrm{P}^{h, \tau}\right)$, we are only able to prove existence of a solution to $\left(\widetilde{\mathrm{P}}^{h, \tau}\right)$ in the case $N=2$, see Barrett et al. [8, Sect. 4]. Here we will only consider the regularised approximation $\left(\mathrm{P}_{\sigma}^{h, \tau}\right)$ in which case, on recalling $(2.36), \widetilde{\boldsymbol{V}}^{h}\left(\boldsymbol{q}^{h}\right) \equiv \boldsymbol{S}^{h}$. Well-posedness of $\left(\widetilde{\mathrm{P}}_{\sigma}^{h, \tau}\right)$ is proved via regularisation. Moreover, one can show that $\left(\widetilde{\mathrm{P}}_{\sigma}^{h, \tau}\right)$ has the property that $\boldsymbol{U}_{\sigma}^{0}>0$ implies $\boldsymbol{U}_{\sigma}^{k}>0$ for all $k \geq 1$. The free energy component $\psi_{1}(\cdot)$ is replaced by the twice continuously differentiable function

$$
\psi_{1, \varepsilon}(r):= \begin{cases}\psi_{1}(r) \equiv \theta r \ln r & \varepsilon \leq r \\ \theta r \ln \varepsilon+\frac{\theta}{2 \varepsilon}\left(r^{2}-\varepsilon^{2}\right) & r \leq \varepsilon\end{cases}
$$


where $\varepsilon \in(0,1]$. Let us emphasize that we introduce $\psi_{1, \varepsilon}$ only to prove well-posedness of problem $\left(\widetilde{\mathrm{P}}_{\sigma}^{h, \tau}\right)$. In practice we solve $\left(\widetilde{\mathrm{P}}_{\sigma}^{h, \tau}\right)$ directly. The monotone function

$$
\psi_{1, \varepsilon}^{\prime}(r)=\left\{\begin{array}{ll}
\theta[\ln r+1] & \varepsilon \leq r \\
\theta\left[\ln \varepsilon+\varepsilon^{-1} r\right] & r \leq \varepsilon
\end{array},\right.
$$

has the properties:

$$
\begin{aligned}
\psi_{1}^{\prime}(r) & \leq \psi_{1, \varepsilon}^{\prime}(r) \quad \forall r>0, \\
\left(\psi_{1, \varepsilon}^{\prime}(r)-\psi_{1, \varepsilon}^{\prime}(s)\right)^{2} & \leq \psi_{1, \varepsilon}^{\prime \prime}(\min \{r, s\})\left(\psi_{1, \varepsilon}^{\prime}(r)-\psi_{1, \varepsilon}^{\prime}(s)\right)(r-s) \\
& \leq \theta \varepsilon^{-1}\left(\psi_{1, \varepsilon}^{\prime}(r)-\psi_{1, \varepsilon}^{\prime}(s)\right)(r-s) \quad \forall r, s .
\end{aligned}
$$

We then introduce $\Psi_{\varepsilon} \in C^{1}\left(\mathbb{R}^{N}\right)$ defined by

$$
\Psi_{\varepsilon}(\boldsymbol{\zeta}):=\Psi_{1, \varepsilon}(\boldsymbol{\zeta})-\frac{1}{2} \boldsymbol{\zeta}^{T} A \boldsymbol{\zeta}, \quad \text { where } \quad \Psi_{1, \varepsilon}(\boldsymbol{\zeta}):=\sum_{n=1}^{N} \psi_{1, \varepsilon}\left(\zeta_{n}\right)
$$

It is a simple matter, see Barrett and Blowey [2, p. 263], to show that $\Psi_{\varepsilon}$ is bounded below for $\varepsilon \leq \varepsilon_{0}:=$ $\min \left\{1, \theta /\left(4 N(N-1)^{2} \lambda_{A \max }\right)\right\}$ and $\theta \leq \theta_{\max }$; that is,

$$
\Psi_{\varepsilon}(\boldsymbol{\zeta}) \geq \frac{\theta}{4 \varepsilon} \sum_{n=1}^{N}\left[\zeta_{n}\right]_{-}^{2}-C\left(N, \theta_{\max }, \lambda_{A \max }\right) \quad \forall \boldsymbol{\zeta} \in \mathcal{M}(1),
$$

where $[\cdot]_{-}:=\min \{\cdot, 0\}$ and $\lambda_{A \max }$ is the largest positive eigenvalue of $A$. We introduce also the strictly convex energy functional $\Psi_{\varepsilon}^{+} \in C^{1}\left(\mathbb{R}^{N}\right)$ defined by

$$
\Psi_{\varepsilon}^{+}(\boldsymbol{\zeta}):=\Psi_{1, \varepsilon}(\boldsymbol{\zeta})-\frac{1}{2} \boldsymbol{\zeta}^{T} A^{-} \boldsymbol{\zeta}
$$

In order to prove well-posedness of $\left(\widetilde{\mathrm{P}}_{\sigma}^{h, \tau}\right)$, we require the assumptions $(\widetilde{\mathrm{A}})$ on the mesh. It follows from (4.6b) and $(\widetilde{\mathrm{A}})$ for all $\varepsilon \leq 1$ and for all $\kappa \in \mathcal{T}^{h}$ that

$$
\begin{aligned}
\int_{\kappa}\left|\nabla \pi^{h}\left[\psi_{1, \varepsilon}^{\prime}(\chi)\right]\right|^{2} \mathrm{~d} \boldsymbol{x} & \leq \psi_{1, \varepsilon}^{\prime \prime}\left(\min _{\boldsymbol{x} \in \bar{\kappa}} \chi(\boldsymbol{x})\right) \int_{\kappa} \nabla \chi \cdot \nabla \pi^{h}\left[\psi_{1, \varepsilon}^{\prime}(\chi)\right] \mathrm{d} \boldsymbol{x} \\
& \leq \frac{\theta}{\varepsilon} \int_{\kappa} \nabla \chi \cdot \nabla \pi^{h}\left[\psi_{1, \varepsilon}^{\prime}(\chi)\right] \mathrm{d} \boldsymbol{x} \quad \forall \chi \in S^{h},
\end{aligned}
$$

see for example Cialvaldini [13].

Theorem 4.1. Let $\Omega$ and $\mathcal{T}^{h}$ be such that assumption $(\widetilde{A})$ hold and let $\boldsymbol{U}_{\sigma}^{0} \in \boldsymbol{K}_{m}^{h}$. In addition let $L^{\sigma}$ satisfy (2.4a,b). Then for all $h>0$ and all time partitions $\left\{\tau_{k}\right\}_{k=1}^{K}$ there exists a unique solution $\left\{\boldsymbol{U}_{\sigma}^{k}, \boldsymbol{W}_{\sigma}^{k}\right\}_{k=1}^{K}$ to $\left(\widetilde{P}_{\sigma}^{h, \tau}\right)$. Furthermore, the stability bounds (2.30) hold.

Proof. Given $\boldsymbol{U}_{\sigma}^{k-1} \in \boldsymbol{K}_{\boldsymbol{m}}^{h}$ with $\left|\boldsymbol{U}_{\sigma}^{k-1}\right|_{1} \leq C$, we prove existence of $\left\{\boldsymbol{U}_{\sigma}^{k}, \boldsymbol{W}_{\sigma}^{k}\right\}$ solving (4.2a,b) by introducing a regularised version

Find $\left\{\boldsymbol{U}_{\sigma, \varepsilon}^{k}, \boldsymbol{W}_{\sigma, \varepsilon}^{k}\right\} \in \boldsymbol{S}^{h} \times \boldsymbol{S}^{h}$ such that

$$
\begin{gathered}
\left(\frac{\boldsymbol{U}_{\sigma, \varepsilon}^{k}-U_{\sigma}^{k-1}}{\Delta t}, \boldsymbol{\chi}\right)^{h}+\left(L^{\sigma}\left(\boldsymbol{U}_{\sigma}^{k-1}\right) \nabla \boldsymbol{W}_{\sigma, \varepsilon}^{k}, \nabla \boldsymbol{\chi}\right)=0 \quad \forall \boldsymbol{\chi} \in \boldsymbol{S}^{h}, \\
\gamma\left(\nabla \boldsymbol{U}_{\sigma, \varepsilon}^{k}, \nabla \boldsymbol{\chi}\right)+\left(\boldsymbol{D} \Psi_{1, \varepsilon}\left(\boldsymbol{U}_{\sigma, \varepsilon}^{k}\right)-A^{-} \boldsymbol{U}_{\sigma, \varepsilon}^{k}, \boldsymbol{\chi}\right)^{h}=\left(\boldsymbol{W}_{\sigma, \varepsilon}^{k}+A^{+} \boldsymbol{U}_{\sigma}^{k-1}, \boldsymbol{\chi}\right)^{h} \quad \forall \boldsymbol{\chi} \in \boldsymbol{S}^{h} .
\end{gathered}
$$


Similarly to (2.28), we have that

$$
\boldsymbol{W}_{\sigma, \varepsilon}^{k} \equiv-\hat{\mathcal{G}}_{\sigma, \boldsymbol{U}_{\sigma}^{k-1}}^{h}\left(\frac{\boldsymbol{U}_{\sigma, \varepsilon}^{k}-\boldsymbol{U}_{\sigma}^{k-1}}{\tau_{k}}\right)+\left(f+(I-f) \mathbf{1} \sum\right) \boldsymbol{\pi}^{h}\left[\boldsymbol{D} \Psi_{1, \varepsilon}\left(\boldsymbol{U}_{\sigma, \varepsilon}^{k}\right)-\left(A^{-} \boldsymbol{U}_{\sigma, \varepsilon}^{k}+A^{+} \boldsymbol{U}_{\sigma}^{k-1}\right)\right] .
$$

Hence (4.11a,b) can be restated as: Find $\boldsymbol{U}_{\sigma, \varepsilon}^{k} \in \boldsymbol{S}^{h}$ such that $f \boldsymbol{U}_{\sigma, \varepsilon}^{k}=f \boldsymbol{u}^{0}$ and

$$
\begin{gathered}
\gamma\left(\nabla \boldsymbol{U}_{\sigma, \varepsilon}^{k}, \nabla \boldsymbol{\chi}\right)+\left(\left(I-\mathbf{1} \sum\right)\left(\boldsymbol{D} \Psi_{1, \varepsilon}\left(\boldsymbol{U}_{\sigma, \varepsilon}^{k}\right)-\left(A^{-} \boldsymbol{U}_{\sigma, \varepsilon}^{k}+A^{+} \boldsymbol{U}_{\sigma}^{k-1}\right)\right),(I-f) \boldsymbol{\chi}\right)^{h} \\
+\left(\hat{\mathcal{G}}_{\sigma, \boldsymbol{U}_{\sigma}^{k-1}}^{h}\left(\frac{\boldsymbol{U}_{\sigma, \varepsilon}^{k}-\boldsymbol{U}_{\sigma}^{k-1}}{\tau_{k}}\right), \boldsymbol{\chi}\right)^{h}=0 \quad \forall \boldsymbol{\chi} \in \boldsymbol{S}^{h} .
\end{gathered}
$$

Existence of $\left\{\boldsymbol{U}_{\sigma, \varepsilon}^{k}, \boldsymbol{W}_{\sigma, \varepsilon}^{k}\right\}$ and uniqueness of $\boldsymbol{U}_{\sigma, \varepsilon}^{k}$ then follows as for $\left\{\boldsymbol{U}_{\sigma}^{k}, \boldsymbol{W}_{\sigma}^{k}\right\}$ in the proof of Theorem 2.1. Uniqueness of $\boldsymbol{W}_{\sigma, \varepsilon}^{k}$ follows immediately from (4.11b) and the uniqueness of $\boldsymbol{U}_{\sigma, \varepsilon}^{k}$. Similarly to (2.32), on noting the convexity of $\psi_{1, \varepsilon}$, the semi positive definiteness of $A^{+},-A^{-}$and the assumptions on $\boldsymbol{U}_{\sigma}^{k-1}$, we have that $\boldsymbol{U}_{\sigma, \varepsilon}^{k}-\boldsymbol{U}_{\sigma}^{k-1} \in \boldsymbol{V}^{h}$ is such that

$$
\begin{gathered}
\frac{\gamma}{2}\left|\boldsymbol{U}_{\sigma, \varepsilon}^{k}\right|_{1}^{2}+\frac{\gamma}{2}\left|\boldsymbol{U}_{\sigma, \varepsilon}^{k}-\boldsymbol{U}_{\sigma}^{k-1}\right|_{1}^{2}+\left(\Psi_{\varepsilon}\left(\boldsymbol{U}_{\sigma, \varepsilon}^{k}\right), 1\right)^{h}+\tau_{k}\left|\left[L^{\sigma}\left(\boldsymbol{U}_{\sigma}^{k-1}\right)\right]^{\frac{1}{2}} \nabla \boldsymbol{W}_{\sigma, \varepsilon}^{k}\right|_{0}^{2} \\
\leq\left(\Psi_{\varepsilon}\left(\boldsymbol{U}_{\sigma}^{k-1}\right), 1\right)^{h}+\frac{\gamma}{2}\left|\boldsymbol{U}_{\sigma}^{k-1}\right|_{1}^{2} \leq C .
\end{gathered}
$$

From (4.14) and (4.8) we deduce that

$$
\left|\left[\boldsymbol{U}_{\sigma, \varepsilon}^{k}\right]_{-}\right|_{h}^{2} \leq C \varepsilon
$$

On setting $\boldsymbol{\chi} \equiv\left(I-\mathbf{1} \sum\right) \boldsymbol{\pi}^{h}\left[\boldsymbol{D} \Psi_{1, \varepsilon}\left(\boldsymbol{U}_{\sigma, \varepsilon}^{k}\right)\right]$ in (4.13), we have on noting (4.10), (4.6b), (2.15), (1.18), (2.7), (4.14) and (4.8) that

$$
\begin{aligned}
\varepsilon\left|\boldsymbol{\pi}^{h}\left[\boldsymbol{D} \Psi_{1, \varepsilon}\left(\boldsymbol{U}_{\sigma, \varepsilon}^{k}\right)\right]\right|_{1}^{2} & +\left|\left(I-\mathbf{1} \sum\right)(I-f) \boldsymbol{\pi}^{h}\left[\boldsymbol{D} \Psi_{1, \varepsilon}\left(\boldsymbol{U}_{\sigma, \varepsilon}^{k}\right)\right]\right|_{h}^{2} \\
& \leq C\left[\left|\boldsymbol{U}_{\sigma, \varepsilon}^{k}\right|_{0}^{2}+\left|\boldsymbol{U}_{\sigma}^{k-1}\right|_{0}^{2}+\sigma^{-2}\left|\left[L\left(\boldsymbol{U}_{\sigma}^{k-1}\right)\right]^{\frac{1}{2}} \nabla \hat{\mathcal{G}}_{\sigma, \boldsymbol{U}_{\sigma}^{k-1}}^{h}\left(\frac{\boldsymbol{U}_{\sigma, \varepsilon}^{k}-\boldsymbol{U}_{\sigma}^{k-1}}{\tau_{k}}\right)\right|_{0}^{2}\right] \\
& \leq C \tau_{k}^{-1} \sigma^{-2} .
\end{aligned}
$$

Choosing $\boldsymbol{\chi} \equiv \boldsymbol{U}_{\sigma, \varepsilon}^{k}$ in (4.13) and noting (4.9), it follows for any constant $\boldsymbol{\zeta} \in \mathbb{R}^{N}$ that

$$
\begin{aligned}
& \left(\boldsymbol{D} \Psi_{\varepsilon}^{+}\left(\boldsymbol{U}_{\sigma, \varepsilon}^{k}\right), \boldsymbol{\zeta}-f \boldsymbol{U}_{\sigma, \varepsilon}^{k}\right)^{h} \leq\left(\Psi_{\varepsilon}^{+}(\boldsymbol{\zeta})-\Psi_{\varepsilon}^{+}\left(\boldsymbol{U}_{\sigma, \varepsilon}^{k}\right), 1\right)^{h} \\
& \quad+\left(A^{+} \boldsymbol{U}_{\sigma}^{k-1}-\hat{\mathcal{G}}_{\sigma, U_{\sigma}^{k-1}}^{h}\left(\frac{\boldsymbol{U}_{\sigma, \varepsilon}^{k}-U_{\sigma}^{k-1}}{\tau_{k}}\right),(I-f) \boldsymbol{U}_{\sigma, \varepsilon}^{k}\right)^{h} .
\end{aligned}
$$

Choosing $\boldsymbol{\zeta} \equiv\left(f \boldsymbol{U}_{\sigma, \varepsilon}^{k}\right) \pm m_{n} \boldsymbol{e}^{n}, n=1 \rightarrow N$, in (4.17); noting that $f \boldsymbol{U}_{\sigma, \varepsilon}^{k}=\boldsymbol{m},(4.9),(4.8),(1.18),(2.7)$ and (4.16) it follows that

$$
\begin{aligned}
\text { If }\left.\boldsymbol{\pi}^{h}\left[\boldsymbol{D} \Psi_{1, \varepsilon}\left(\boldsymbol{U}_{\sigma, \varepsilon}^{k}\right)\right]\right|_{h} ^{2} & \leq C\left[1+\sigma^{-2}\left|\left[L\left(\boldsymbol{U}_{\sigma}^{k-1}\right)\right]^{\frac{1}{2}} \nabla \hat{\mathcal{G}}_{\sigma, \boldsymbol{U}_{\sigma}^{k-1}}^{h}\left(\frac{\boldsymbol{U}_{\sigma, \varepsilon}^{k}-\boldsymbol{U}_{\sigma}^{k-1}}{\tau_{k}}\right)\right|_{0}^{2}\right] \\
& \leq C \tau_{k}^{-1} \sigma^{-2} .
\end{aligned}
$$

Noting that $N \sum \boldsymbol{U}_{\sigma, \varepsilon}^{k}=1$ and the monotonicity of $\psi_{1, \varepsilon}^{\prime}(\cdot)$, it follows that

$$
\left|\psi_{1, \varepsilon}^{\prime}\left(\frac{1}{N}\right)-\sum \boldsymbol{\pi}^{h}\left[\boldsymbol{D} \Psi_{1, \varepsilon}\left(\boldsymbol{U}_{\sigma, \varepsilon}^{k}\right)\right]\right|_{h}^{2} \leq\left|\left(I-\mathbf{1} \sum\right) \boldsymbol{\pi}^{h}\left[\boldsymbol{D} \Psi_{1, \varepsilon}\left(\boldsymbol{U}_{\sigma, \varepsilon}^{k}\right)\right]\right|_{h}^{2} .
$$


Hence combining (4.16), (4.18) and (4.19) we have for $\varepsilon \leq \min \left\{\varepsilon_{0}, \frac{1}{N}\right\}$ that

$$
\tau_{k}\left|\boldsymbol{\pi}^{h}\left[\boldsymbol{D} \Psi_{1, \varepsilon}\left(\boldsymbol{U}_{\sigma, \varepsilon}^{k}\right)\right]\right|_{h}^{2} \leq C \sigma^{-2}
$$

It follows from (4.14), (4.8) and $\boldsymbol{U}_{\sigma, \varepsilon}^{k}-\boldsymbol{U}_{\sigma}^{k-1} \in \boldsymbol{V}^{h}$ that there exists a $\boldsymbol{U}_{\sigma}^{k}$ and a subsequence $\left\{\boldsymbol{U}_{\sigma, \varepsilon^{\prime}}^{k}\right\}$ such that $\boldsymbol{U}_{\sigma}^{k}-\boldsymbol{U}_{\sigma}^{k-1} \in \boldsymbol{V}^{h}$ and $\boldsymbol{U}_{\sigma, \varepsilon^{\prime}}^{k} \rightarrow \boldsymbol{U}_{\sigma}^{k}$ as $\varepsilon^{\prime} \rightarrow 0$. It follows from (4.20) that there exists $\boldsymbol{\phi}^{h, k} \in \boldsymbol{S}^{h}$ such that $\boldsymbol{\pi}^{h}\left[\boldsymbol{D} \Psi_{1, \varepsilon^{\prime}}\left(\boldsymbol{U}_{\sigma, \varepsilon^{\prime}}^{k}\right)\right] \rightarrow \boldsymbol{\phi}^{h, k}$ as $\varepsilon^{\prime} \rightarrow 0$. Noting that for all $s \in \mathbb{R},\left[\psi_{1, \varepsilon}^{\prime}\right]^{-1}(s) \rightarrow\left[\psi_{1}^{\prime}\right]^{-1}(s)$ as $\varepsilon \rightarrow 0$ we have that $\boldsymbol{\phi}^{h, k} \equiv \boldsymbol{\pi}^{h}\left[\boldsymbol{D} \Psi_{1}\left(\boldsymbol{U}_{\sigma}^{k}\right)\right]$. Therefore we may pass to the limit $\varepsilon^{\prime} \rightarrow 0$ in (4.13) to prove existence of a solution to $\left(\widetilde{\mathrm{P}}_{\sigma}^{h, \tau}\right)$ at time level $t_{k}$ on noting (4.12). Uniqueness of $\boldsymbol{U}_{\sigma}^{k}$ follows from the monotonicity of $\psi_{1}$. Hence noting (4.12), we have existence and uniqueness of a solution $\left\{\boldsymbol{U}_{\sigma}^{k}, \boldsymbol{W}_{\sigma}^{k}\right\}$ to $\left(\widetilde{\mathrm{P}}_{\sigma}^{h, \tau}\right)$ at time level $t_{k}$. As the bound (4.20) holds with $\Psi_{1, \varepsilon}\left(\boldsymbol{U}_{\sigma, \varepsilon}^{k}\right)$ replaced by $\Psi_{1}\left(\boldsymbol{U}_{\sigma}^{k}\right)$, it follows that $\boldsymbol{U}_{\sigma}^{k}>\mathbf{0}$. As the bound (4.14) holds with the subscript " $\varepsilon$ " removed, the stability bounds (2.30) follow as in the proof of Theorem 2.1.

Adopting the notation (2.50a,b) for the solution $\left\{\boldsymbol{U}_{\sigma}^{k}, \boldsymbol{W}_{\sigma}^{k}\right\}_{k=1}^{K}$ of $\left(\widetilde{\mathrm{P}}_{\sigma}^{h, \tau}\right)$, we have the analogue of Theorem 2.2.

Theorem 4.2. Let the assumptions of Theorem 2.2 hold with (A) replaced by $(\widetilde{A})$, and now in particular with $\psi_{1}$ assumed to be of the logarithmic form (4.1). Then there exists a subsequence of solutions $\left\{\boldsymbol{U}_{\sigma}, \boldsymbol{W}_{\sigma}\right\}_{h}$ of problem $\left(\widetilde{P}_{\sigma}^{h, \tau}\right)$ and a function $\boldsymbol{u} \in L^{\infty}(0, T ; \boldsymbol{K}) \cap H^{1}\left(0, T ;\left(\boldsymbol{H}^{1}(\Omega)\right)^{\prime}\right) \cap \boldsymbol{C}_{x, t}^{\frac{1}{2}, \frac{1}{8}}\left(\bar{\Omega}_{T}\right)$ and functions $z_{n p} \in$ $L_{l o c}^{2}\left(\left\{u_{n}>0\right\} \cap\left\{u_{p}>0\right\}\right), n, p=1 \rightarrow N$ with $\frac{\partial}{\partial x} z_{n p} \in L_{l o c}^{2}\left(\left\{u_{n}>0\right\} \cap\left\{u_{p}>0\right\}\right)$ such that as $h \rightarrow 0$ (2.54-2.56) and (2.5\%a,b) hold.

Proof. The proof is the same as that of Theorem 2.2 with the following minor changes. We only mention the modifications caused by the presence of the logarithmic free energy which implies that $\psi_{1}^{\prime}$ becomes unbounded. Clearly the inequality, the test function $\boldsymbol{\chi}-\boldsymbol{U}_{\sigma}^{+}$and $\boldsymbol{K}^{h}$ in $(2.53 \mathrm{~b})$ are replaced by equality, $\boldsymbol{\chi}$ and $\boldsymbol{S}^{h}$, respectively. Although (2.74) is redundant, (2.75) still holds on noting the above. It follows from (2.78) and (2.71) that $C\left\|\alpha_{\delta, n p} Z_{(\sigma,) n p}^{+}\right\|_{L^{2}\left(\Omega_{T}\right)}$ on the right hand side of the first inequality in (2.79) is replaced by $C\left(\delta^{-1}\right)\left\|\alpha_{\delta, n p} Z_{(\sigma,) n p}^{+}\right\|_{L^{2}\left(\Omega_{T}\right)}$ with the final bound of (2.79) remaining the same. Clearly (2.87) remains true for all $\eta_{n} \in L^{2}\left(0, T ; H^{1}(\Omega)\right)$ with $\operatorname{supp}\left(\eta_{n}\right) \subset D_{\delta, n}^{+}$on noting the technique used in (2.86). Hence (2.89) remains true.

Theorem 4.3. We consider the approximation $\left(P_{\sigma}^{h, \tau}\right)$ with the mobility matrix $L^{\sigma}$ replaced by $\widehat{L}^{\sigma}$ and $\psi_{1}$ of the logarithmic form (4.1). Then the results of Theorems 2.1 and 2.2 remain true under their stated assumptions with $(A)$ replaced by $(\widetilde{A})$ and $L^{\sigma}$ replaced by $\widehat{L}^{\sigma}$.

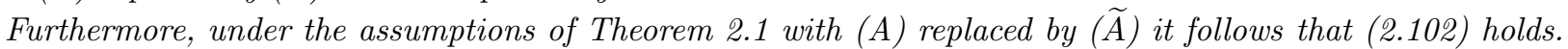

Moreover, under the assumptions of Theorem 2.2 with the additional constraint that $\tau \leq C \sigma^{2}$ as $h \rightarrow 0$ we have that the limit function $\boldsymbol{u} \in L^{2}\left(0, T ; \boldsymbol{H}^{2}(\Omega)\right)$.

Proof. The proof is exactly the same as that of Theorem 2.3 except for (2.107), which we replace by the following. From (2.100), (2.106) and $\boldsymbol{U}_{\sigma}^{k} \in \boldsymbol{K}_{\boldsymbol{m}}^{h}$ with $\boldsymbol{U}_{\sigma}^{k}>\mathbf{0}$ we have for $n=1 \rightarrow N$ that

$$
\begin{aligned}
\gamma\left|\Delta^{h} U_{\sigma, n}^{k}\right|_{h}^{2}=-\gamma\left(\nabla U_{\sigma, n}^{k}, \nabla\left(\Delta^{h} U_{\sigma, n}^{k}\right)\right) & \leq-\left(W_{\sigma, n}^{k}, \Delta^{h} U_{\sigma, n}^{k}\right)^{h}+\left(\psi_{1}^{\prime}\left(U_{\sigma, n}^{k}\right), \Delta^{h} U_{\sigma, n}^{k}\right)^{h}+C \\
& =\left(\nabla W_{\sigma, n}^{k}, \nabla U_{\sigma, n}^{k}\right)-\left(\nabla \pi^{h}\left[\psi_{1}^{\prime}\left(U_{\sigma, n}^{k}\right)\right], \nabla U_{\sigma, n}^{k}\right)+C \\
& \leq\left(\nabla W_{\sigma, n}^{k}, \nabla U_{\sigma, n}^{k}\right)+C
\end{aligned}
$$

on noting, similarly to (4.10), that for all $\kappa \in \mathcal{T}^{h}$

$$
\int_{\kappa} \nabla \chi \cdot \nabla \pi^{h}\left[\psi_{1}^{\prime}(\chi)\right] \mathrm{d} \boldsymbol{x} \geq 0 \quad \forall \chi \in S^{h} \text { with } \chi>0
$$


A remark similar to that after Theorem 2.3 also applies in the logarithmic case for $d \geq 2$. Finally we modify the iterative algorithm in Section 3, to solve the nonlinear algebraic system for $\left\{\boldsymbol{U}_{\sigma}^{k}, \boldsymbol{W}_{\sigma}^{k}\right\}$ arising in $\left(\widetilde{\mathrm{P}}_{\sigma}^{h, \tau}\right)$ with $L^{\sigma}$ or $\widehat{L}^{\sigma}$. We have that $\left\{\boldsymbol{U}_{\sigma}^{k}, \boldsymbol{W}_{\sigma}^{k}\right\} \in \boldsymbol{S}^{h} \times \boldsymbol{S}^{h}$ satisfy

$$
\left(\boldsymbol{U}_{\sigma}^{k}+\mu \boldsymbol{D} \Psi_{1}\left(\boldsymbol{U}_{\sigma}^{k}\right), \boldsymbol{\chi}\right)^{h}=\left(\boldsymbol{R}^{k}, \boldsymbol{\chi}\right)^{h} \quad \forall \boldsymbol{\chi} \in \boldsymbol{S}^{h}
$$

in place of $(3.2 \mathrm{a})$ with $(3.2 \mathrm{~b}-\mathrm{d})$ remaining the same. Hence we modify our iterative procedure (3.3a-d) by replacing (3.3b) by: find $\boldsymbol{U}^{n, p+\frac{1}{2}} \in S^{h}$ such that for $n=1 \rightarrow N$

$$
U_{n}^{k, p+\frac{1}{2}}\left(\boldsymbol{x}_{j}\right)+\mu \psi_{1}^{\prime}\left(U_{n}^{k, p+\frac{1}{2}}\left(\boldsymbol{x}_{j}\right)\right)=R_{n}^{k, p}\left(\boldsymbol{x}_{j}\right) \quad \forall j \in J
$$

and keeping $(3.3 \mathrm{a}, \mathrm{c}, \mathrm{d})$ the same. Existence and uniqueness of $U_{n}^{k, p+\frac{1}{2}}\left(\boldsymbol{x}_{j}\right)$ follows from the monotonicity of $\psi_{1}^{\prime}(\cdot)$. Hence this modified iterative procedure is well-defined. Moreover, Theorem 3.1 still holds.

\section{NUMERICAL EXPERIMENTS}

All computations were performed in double precision on a Sparc 20. The program was written in Fortran 77 using the NAG subroutine C06HBF for calculating the discrete cosine transform. In all experiments we compute the solution to $\left(\mathrm{P}_{(\sigma)}^{h, \Delta t}\right)$ where $\Psi_{1}$ is given by $(1.8 \mathrm{~b}), N=3$ and $L^{(\sigma)}$ given by $(2.4 \mathrm{a}, \mathrm{b}),(1.6)$ and $l_{n}(s) \equiv s$, $n=1 \rightarrow N$.

\subsection{One space dimension}

The computations were performed on a uniform partitioning of $\Omega=(0,1)$ with mesh points $x_{j}=(j-1) h$, $j=1 \rightarrow \# J$, where $h=1 /(\# J-1)$. We note that the integral on the right hand side of (3.4) can be evaluated exactly using Simpson's rule since the entries of $L^{(\sigma)}(\cdot)$ are quadratic.

\section{Experiment 1.}

The aim of this experiment is two-fold. Firstly we demonstrate the fundamental difference between a nondegenerate and a degenerate mobility matrix. Note that even though we have been unable to prove existence, we have not experienced any difficulty in computing the approximation $\left(\mathrm{P}^{h, \tau}\right)$. Secondly we show that for $\sigma$ small and positive there is no significant difference between the numerical approximations $\left(\mathrm{P}_{\sigma}^{h, \tau}\right)$ and $\left(\mathrm{P}^{h, \tau}\right)$. We set $\gamma=3.2 \times 10^{-3}$,

$$
A=-\left(\begin{array}{lll}
0 & 1 & 1 \\
1 & 0 & 1 \\
1 & 1 & 0
\end{array}\right) \Longrightarrow A^{-}=-\frac{2}{3}\left(\begin{array}{lll}
1 & 1 & 1 \\
1 & 1 & 1 \\
1 & 1 & 1
\end{array}\right), \quad A^{+}=\frac{1}{3}\left(\begin{array}{ccc}
2 & -1 & -1 \\
-1 & 2 & -1 \\
-1 & -1 & 2
\end{array}\right)
$$

$T=6.4, \sigma=0.1, L_{\max }^{\sigma}=\frac{1}{2}(1+3 \sigma), \# J=101$, uniform $\tau=1.6 \times 10^{-3}, \mu=0.02$ and took as our stopping criterion: $\max _{n=1 \rightarrow 3}\left\|U_{(\sigma,) n}^{k, p}-U_{(\sigma,) n}^{k, p-1}\right\|_{0, \infty} \leq$ tol $:=10^{-8}$. We started with a stationary numerical solution (that is the stopping criterion for the iterative procedure is satisfied in a single step from one time level to the next) from an experiment where $\sigma=0$ and $\boldsymbol{m} \approx(0.48,0.48,0.04)$. We plot the resulting evolution in Figure 1 . At $t=2.56$ the solution is graphically no different to the corresponding stationary numerical solution, which was obtained by continuing the experiment further in time.

Fixing the same data as above, we chose $\sigma=10^{-1}, 10^{-2}, 10^{-3}, 10^{-4}, 10^{-5}, 10^{-6}$ and 0 . Obviously with 

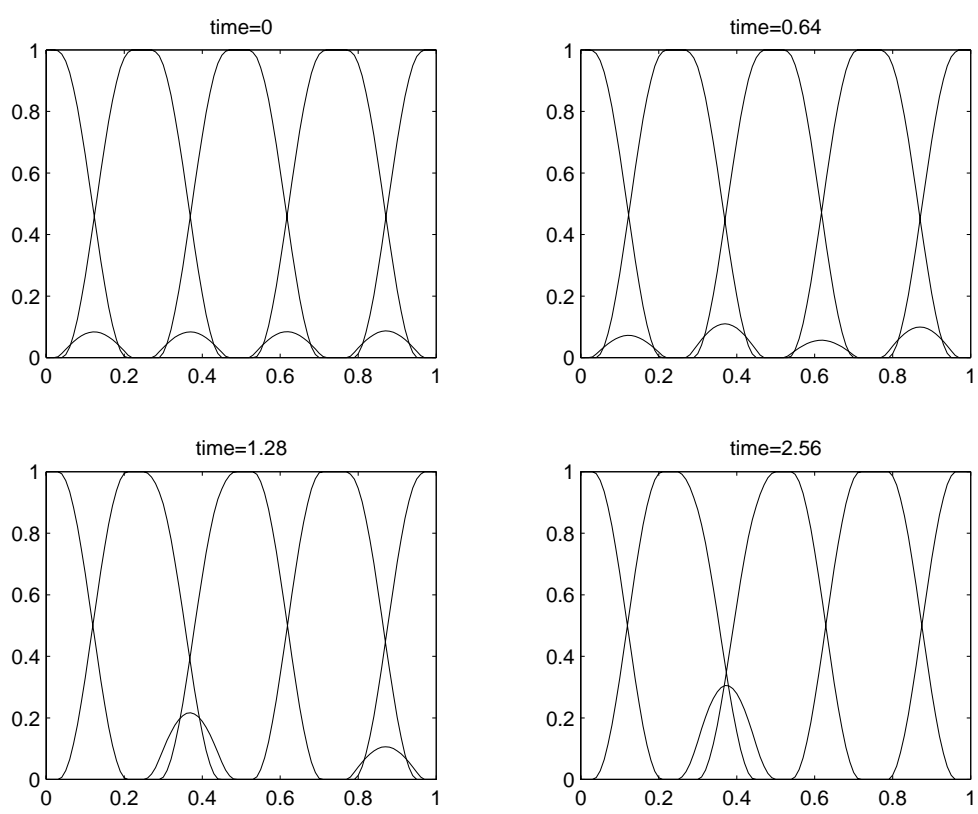

Figure 1. Non-degenerate mobility.

$\sigma=0$ we see no evolution from Figure 1 when $t=0$. In the table below we make a comparison between the resulting solution at $t=6.4$ and the average number of iterations required for the algorithm to converge over the last three time steps.

$\begin{array}{ccc}\sigma & \left\|\boldsymbol{U}_{\sigma}(\cdot, 6.4)-\boldsymbol{U}^{0}(\cdot)\right\|_{0, \infty} & \text { number of iterations } \\ 0 & 0 & 1 \\ 10^{-6} & 3 \times 10^{-5} & 1 \\ 10^{-5} & 1 \times 10^{-5} & 1 \\ 10^{-4} & 4 \times 10^{-4} & 7 \\ 10^{-3} & 4 \times 10^{-3} & 23 \\ 10^{-2} & 9 \times 10^{-2} & 46 \\ 10^{-1} & 0.13 & 30\end{array}$

By adapting an argument in Barrett and Blowey [3], see for instance page 29 where two bumps are present in one component, it is possible to construct continuous stationary solutions with four bumps in one component. For $\sigma=0$ the heights of the bumps are not required to be equal, whereas for $\sigma>0$ the heights of the bumps have to be equal. Moreover, with $\sigma=10^{-4}, 10^{-5}$ and $10^{-6}$ the graphs of $\boldsymbol{U}_{\sigma}(\cdot, 6.4)$ and $\boldsymbol{U}^{0}(\cdot)$ are graphically indistinguishable. From the table above we conclude that numerically with $\sigma$ small and positive our computation sees $L^{\sigma}(\cdot)$ as degenerate. We make two further conjectures based on this experiment:

1. For non-degenerate mobility a stationary solution with four bumps of equal height in one component is unstable.

2. For degenerate mobility a stationary solution with four bumps of possibly unequal heights in one component is stable.

\subsection{Two space dimensions}

All of our numerical experiments in two spatial dimensions are performed with a degenerate mobility and $\Omega=(0,1) \times(0,1)$. We took a uniform mesh consisting of squares $e$ of length $h=1 / 256$, each of which was then 

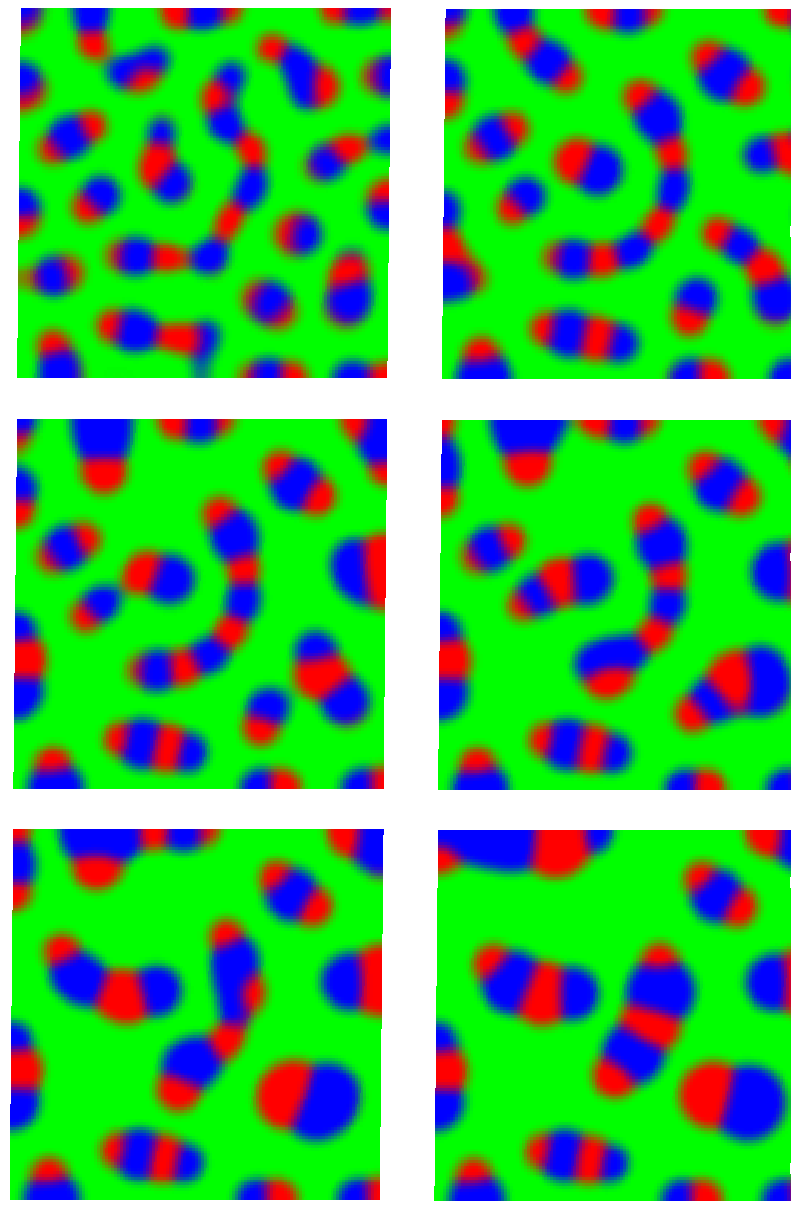

Figure 2. $\boldsymbol{U}(\cdot, t)$ when $t=0.096,0.32,0.64,0.96,1.28$ and $2.88 .^{1}$

subdivided into two triangles by its north east diagonal. We used the following discrete inner product on $C(\bar{\Omega})$

$$
\left(\chi_{1}, \chi_{2}\right)_{\star}^{h}:=\int_{\Omega} \Pi^{h}\left(\chi_{1}(\boldsymbol{x}) \chi_{2}(\boldsymbol{x})\right) \mathrm{d} \boldsymbol{x}
$$

in place of (2.1). Here $\Pi^{h}$ is the piecewise continuous bilinear interpolant on $\Omega$ which is affine linear for $x_{1}$ (or $x_{2}$ ) fixed and interpolates at the vertices on each square $e$. Using (5.1) instead of (2.1) only changes the algorithm at the corners of the square $\Omega$ and has the advantage that one can then solve (3.6a), i.e. (3.9), using "the discrete cosine transform", see [11]. We note that similarly to (2.15) the discrete inner product (5.1) is equivalent to the standard $L^{2}$ inner product. Therefore, it is easy to adapt the proofs to show that the Theorems 2.1, 2.3, 3.1, 4.1 and 4.3 in this paper remain true with this choice of discrete inner product. For the above choice of $l_{n}(s), \boldsymbol{E}^{k, p}$ satisfying (3.4) can be evaluated exactly by sampling at the mid-points of the sides over each triangle $\kappa$. In these numerical results we expect to qualitatively predict the results of [23]. They show that taking (P) with (1.8a) and the singular limit with scaling $\theta=\mathcal{O}(\varepsilon), \gamma=\varepsilon^{2}, t \rightarrow \varepsilon^{2} t$ yields motion by minus the Laplacian of the mean curvature for each of the interfaces. Moreover at triple junctions Young's law,

\footnotetext{
${ }^{1}$ The figure is in color at www.edpsciences.org/docinfos/M2AN
} 
a no-flux condition and a continuity condition for chemical potentials have to hold and at the intersection of an interface with an external boundary a Neumann type angle condition and a no-flux condition are obtained.

\section{Experiment 2.}

We now perform a degenerate mobility numerical experiment with the initial data, $\boldsymbol{U}^{0} \equiv \boldsymbol{m}+\boldsymbol{\delta}^{h}$, where $\boldsymbol{m}=(0.15,0.2,0.65)^{T}$ and $\boldsymbol{\delta}^{h}$ is a random perturbation satisfying $\left\|\delta_{n}^{h}\right\|_{0, \infty} \leq 0.05, n=1 \rightarrow 3$. All the other parameters were the same as in experiment 1, except $\gamma=3.2 \times 10^{-4}, \mu=0.01$ and tol $=10^{-6}$. In Figure 2 the pictures are arranged in a matrix format with time increasing to the right in rows then down columns. The first, second and third components of $\boldsymbol{U}$ consist respectively of the medium, darkest and lightest shade of grey (red, blue and green on the on-line version). We note that the final picture shows a numerical stationary solution.

The main driving force in Cahn-Hilliard systems, both for degenerate and for non-degenerate mobility, is the reduction of the total amount of interfacial area (see e.g. [23] for the case of degenerate mobility and [12] for the case of constant mobility). A main difference between degenerate and non-degenerate mobility is the fact that in the case of degenerate mobility this is done only by local adjustments in connected phase regions, whereas in the case of non-degenerate mobility also non-local interactions are used to achieve this. In the case of degenerate mobility, diffusion takes place only through the narrow interfacial layers. In particular, in Figure 2 there is no diffusion from one chain to the other. Different chains of the two minor phases are pinned in chain like structures and the coarsening process stops, even if there are still many disconnected regions present. The qualitative behaviour of the numerical solution presented in Figure 2 is in accordance with the predictions of the asymptotics in Garcke and Novick-Cohen [23].

In the case of a non-degenerate mobility matrix, diffusion through bulk regions is still possible and disconnected regions influence each other in order to decrease the total amount of interfacial area. For large times, non-degenerate Cahn-Hilliard systems generically lead to situations where each phase occupies only one connected part of the domain (see e.g. [3]).

\section{Experiment 3.}

All the data remained the same as in the previous experiment except we took the stationary numerical solution from experiment 2 as the initial data and set

$$
A=-\left(\begin{array}{lll}
0 & 1 & 8 \\
1 & 0 & 1 \\
8 & 1 & 0
\end{array}\right) \Longrightarrow A^{-}=-\frac{1}{12}\left[\begin{array}{ccc}
24+17 \sqrt{2} & 6+4 \sqrt{2} & 24+17 \sqrt{2} \\
6+4 \sqrt{2} & 2 \sqrt{2} & 6+4 \sqrt{2} \\
24+17 \sqrt{2} & 6+4 \sqrt{2} & 24+17 \sqrt{2}
\end{array}\right]
$$

and

$$
A^{+}=-\frac{1}{12}\left[\begin{array}{ccc}
-24-17 \sqrt{2} & 6-4 \sqrt{2} & 72-17 \sqrt{2} \\
6-4 \sqrt{2} & -2 \sqrt{2} & 6-4 \sqrt{2} \\
72-17 \sqrt{2} & 6-4 \sqrt{2} & -24-17 \sqrt{2}
\end{array}\right]
$$

In Figure 3 after a short period of time the second (dark grey) component surrounds the first (medium grey) component. Note the final picture is not that of a stationary solution. In terms of an energy argument, see $(1.7 \mathrm{a}, \mathrm{b})$, this is understood to occur as the energy required to go directly from the first to the third component is much greater than going via the intermediate second component. This phenomenon is known as wetting. We remark that one phase wetting an interface between two other phases is of course not due to the degenerate mobility matrix. It also occurs in the case of Cahn-Hilliard systems with a non-degenerate mobility matrix and in Allen-Cahn systems (see [22, Fig. 10]). 

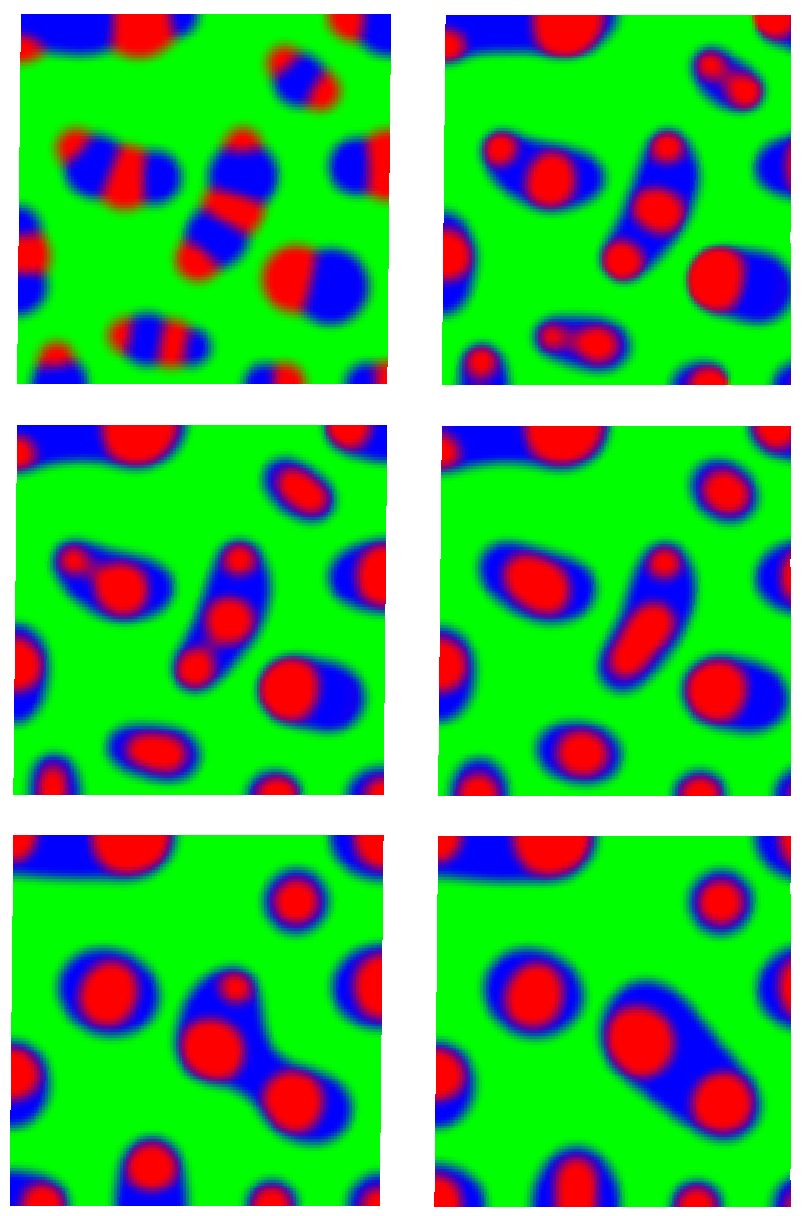

Figure 3. $\boldsymbol{U}(\cdot, t)$ when $t=0,0.032,0.064,0.128,0.832$ and $1.472 .^{1}$

\section{Experiment 4.}

For this experiment, we considered a symmetric situation as in the paper by Ito and Kohsaka [25] who studied the sharp interface problem derived by Garcke and Novick-Cohen [23]. We took the same data as in experiment 2 except

$$
\boldsymbol{U}^{0}\left(x_{i}, y_{j}\right)= \begin{cases}(0,0,1)^{T} & \text { if } 0 \leq x_{i} \leq \frac{13}{16} \text { and } y_{i}>0.65+\frac{\sqrt{3}}{8 \pi} \cos \left(8 \pi x_{i}\right) \\ & \text { or } \frac{13}{16} \leq x_{i} \leq \frac{13}{16}+\frac{0.15}{\sqrt{3}} \text { and } y_{i}>0.65-\sqrt{3}\left(x_{i}-\frac{13}{16}\right) \\ & \text { or } \frac{13}{16}+\frac{0.15}{\sqrt{3}} \leq x_{i} \text { and } y_{i}>\frac{1}{2}, \\ (0,1,0)^{T} & \text { if } 0 \leq x_{i} \leq \frac{13}{16} \text { and } y_{i}<0.35-\frac{\sqrt{3}}{8 \pi} \cos \left(8 \pi x_{i}\right) \\ & \text { or } \frac{13}{16} \leq x_{i} \leq \frac{13}{16}+\frac{0.15}{\sqrt{3}} \text { and } y_{i}<0.35+\sqrt{3}\left(x_{i}-\frac{13}{16}\right) \\ & \text { or } \frac{13}{16}+\frac{0.15}{\sqrt{3}} \leq x_{i} \text { and } y_{i}<\frac{1}{2}, \\ \left(0, \frac{1}{2}, \frac{1}{2}\right)^{T} & \text { if } \frac{13}{16}+\frac{0.15}{\sqrt{3}} \leq x_{i} \text { and } y_{i}=\frac{1}{2}, \\ (1,0,0)^{T} & \text { otherwise. }\end{cases}
$$



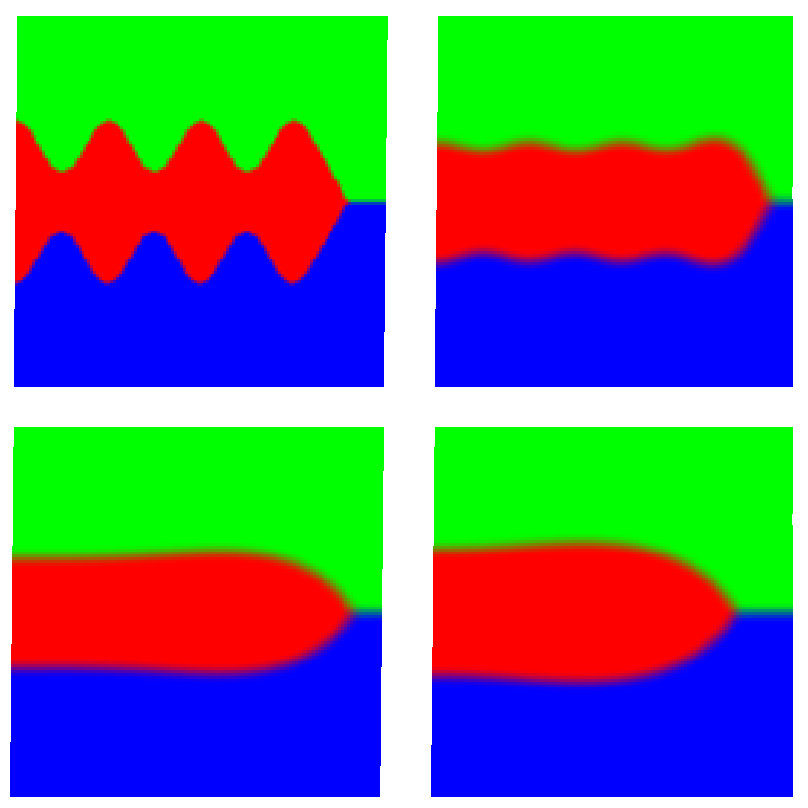

Figure $4 . \boldsymbol{U}(\cdot, t)$ when $t=0,0.32,3.2$ and $27.2 .^{1}$

Notice that the angles at the triple junction in Figure 4 are good approximations to $\frac{2 \pi}{3}$ and the boundary between the 1-2 transition and 1-3 transition meet the external boundary at approximately $\frac{\pi}{2}$. What is expected, and reflected in the numerical experiment, is that the symmetry of the initial data is maintained during the evolution. We did not allow the numerical solution to reach its stationary profile as this would have been an extremely expensive computation but the theoretical results by Ito and Kohsaka [25] indicate that the solution will converge to two arcs and a line segment.

Taking a constant or non-degenerate mobility matrix would lead to similar looking results as those displayed in Figure 4. In this case the limiting sharp interface problem is the multi-phase Mullins-Sekerka evolution introduced in Bronsard et al. [12]. However, a result like the one of [25] is not known so far for this non-local geometric evolution problem.

\section{REFERENCES}

[1] R.A. Adams and J. Fournier, Cone conditions and properties of Sobolev spaces. J. Math. Anal. Appl. 61 (1977) $713-734$.

[2] J.W. Barrett and J.F. Blowey, An error bound for the finite element approximation of a model for phase separation of a multi-component alloy. IMA J. Numer. Anal. 16 (1996) 257-287.

[3] J.W. Barrett and J.F. Blowey, Finite element approximation of a model for phase separation of a multi-component alloy with non-smooth free energy. Numer. Math. 77 (1997) 1-34.

[4] J.W. Barrett and J.F. Blowey, Finite element approximation of a model for phase separation of a multi-component alloy with a concentration dependent mobility matrix. IMA J. Numer. Anal. 18 (1998) 287-328.

[5] J.W. Barrett and J.F. Blowey, Finite element approximation of a model for phase separation of a multi-component alloy with non-smooth free energy and a concentration dependent mobility matrix. $M^{3} A S 9$ (1999) 627-663.

[6] J.W. Barrett and J.F. Blowey, An improved error bound for a finite element approximation of a model for phase separation of a multi-component alloy with a concentration dependent mobility matrix. Numer. Math. 88 (2001) 255-297.

[7] J.W. Barrett, J.F. Blowey and H. Garcke, Finite element approximation of a fourth order nonlinear degenerate parabolic equation. Numer. Math. 80 (1998) 525-556.

[8] J.W. Barrett, J.F. Blowey and H. Garcke, Finite element approximation of the Cahn-Hilliard equation with degenerate mobility. SIAM J. Numer. Anal. 37 (1999) 286-318.

[9] J.F. Blowey, M.I.M. Copetti and C.M. Elliott, The numerical analysis of a model for phase separation of a multi-component alloy. IMA J. Numer. Anal. 16 (1996) 111-139. 
[10] J.F. Blowey and C.M. Elliott, The Cahn-Hilliard gradient theory for phase separation with non-smooth free energy, part i: Mathematical analysis. European J. Appl. Math. 2 (1991) 233-279.

[11] J.F. Blowey and C.M. Elliott, The Cahn-Hilliard gradient theory for phase separation with non-smooth free energy, part ii: Numerical analysis. European J. Appl. Math. 3 (1992) 147-179.

[12] L. Bronsard, H. Garcke and B. Stoth, A multi-phase Mullins-Sekerka system: matched asymptotic expansions and an implicit time discretisation for the geometric evolution problem, in Proc. Roy. Soc. Edinburgh 128 A (1998) 481-506.

[13] J.F. Cialvaldini, Analyse numérique d'un problème de Stefan à deux phases par une méthode d'éléments finis. SIAM J. Numer. Anal. 12 (1975) 464-487.

[14] P.G. Ciarlet, Introduction to numerical linear algebra and optimisation. C.U.P., Cambridge (1988).

[15] D. de Fontaine, An analysis of clustering and ordering in multicomponent solid solutions - I. Stability criteria. J. Phys. Chem. Solids 33 (1972) 297-310.

[16] P.G. de Gennes, Dynamics of fluctuations and spinodal decomposition in polymer blends. J. Chem. Phys. 72 (1980) $4756-4763$.

[17] C.M. Elliott, The Cahn-Hilliard model for the kinetics of phase transitions, in Mathematical models for phase change problems, J.F. Rodrigues Ed., Internat. Ser. Numer. Math. 88, Birkhäuser-Verlag, Basel (1989) 35-73.

[18] C.M. Elliott and H. Garcke, On the Cahn-Hilliard equation with degenerate mobility. SIAM J. Math. Anal. 27 (1996) $404-423$.

[19] C.M. Elliott and H. Garcke, Diffusional phase transitions in multicomponent systems with a concentration dependent mobility matrix. Physica D 109 (1997) 242-256.

[20] C.M. Elliott and S. Luckhaus, A generalized diffusion equation for phase separation of a multi-component mixture with interfacial free energy. SFB256 University Bonn, Preprint 195 (1991).

[21] D.J. Eyre, Systems of Cahn-Hilliard equations. SIAM J. Appl. Math. 53 (1993) 1686-1712.

[22] H. Garcke, B. Nestler and B. Stoth, Anisotropy in multi phase systems: a phase field approach. Interfaces Free Bound. 1 (1999) $175-198$.

[23] H. Garcke and A. Novick-Cohen, A singular limit for a system of degenerate Cahn-Hilliard equations. Adv. Diff. Eq. 5 (2000) 401-434.

[24] G. Grün and M. Rumpf, Nonnegativity preserving numerical schemes for the thin film equation. Numer. Math. 87 (2000) $113-152$.

[25] K. Ito and Y. Kohsaka, Three-phase boundary motion by surface diffusion: stability of a mirror symmetric stationary solution. Interfaces Free Bound. 3 (2001) 45-80.

[26] P.L. Lions and B. Mercier, Splitting algorithms for the sum of two nonlinear operators. SIAM J. Numer. Anal. 16 (1979) 964-979.

[27] J.E. Morral and J.W. Cahn, Spinodal decomposition in ternary systems. Acta Metall. 19 (1971) 1037-1045.

[28] A. Novick-Cohen, The Cahn-Hilliard equation: mathematical and modelling perspectives. Adv. Math. Sci. Appl. 8 (1998) 965-985.

[29] F. Otto and W. E, Thermodynamically driven incompressible fluid mixtures. J. Chem. Phys. 107 (1997) 10177-10184.

[30] L. Zhornitskaya and A.L. Bertozzi, Positivity preserving numerical schemes for lubrication-type equations. SIAM J. Numer. Anal. 37 (2000) 523-555.

To access this journal online:

www.edpsciences.org 